\title{
Capillary Flow in an Interior Corner
}

Mark Milton Weislogel

Lewis Research Center

Cleveland, Ohio

November 1996

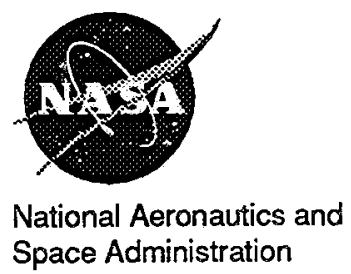

Space Administration 


\title{
ABSTRACT \\ Capillary Flow in an Interior Corner
}

\author{
Mark M. Weislogel
}

The design of fluids management processes in the low-gravity environment of space requires an accurate model and description of capillarity-controlled flow in containers of irregular geometry. Here we consider the redistribution of fluid along an interior corner of a container; following the rapid reduction of gravity, $-g \hat{z}$, the fluid advances along the corner to a height $z=\mathcal{L}(t)$. The analytical portion of the work presents an asymptotic formulation in the limit of a slender fluid column, slight surface curvature along $z$, small inertia, and low gravity. The scaling introduced renders all parameters in closed form or containing a slightly varying coefficient which may be computed numerically. This scaling clarifies the distinction between the effects of corner geometry and surface curvature. Introducing an invariant transformation allows the asymptotic equations to be solved using an accurate and efficient numerical scheme. New similarity solutions are found and a list of closed form expressions is provided for flow rate and column length, $\mathcal{L}$. In particular, it is found that $\mathcal{L}$ is proportional to $t^{1 / 2}$ for the constant height boundary condition, $t^{2 / 5}$ for a spreading drop, and $t^{3 / 5}$ for constant flow. A linear solution which describes the tip of the fluid column is found to exist for all solutions. In the experimental portion of the work, measurements from a $2.2 \mathrm{~s}$ drop tower are reported. An extensive data set, collected over a previously unexplored range of flow parameters, includes estimates of repeatability and accuracy, the role of inertia and column slenderness, and the effects of corner angle, container geometry, and fluid properties. It is found that there exists a location $z_{H}$ at which the interface height remains constant $h\left(z_{H}, t\right)=H$ after an initial transient, $t>t_{H}$. The height $H$ is equal to the value predicted from the static shape of a constant curvature fluid interface. Comprehensive comparison is made between the analysis and measurements using the constant height boundary condition. Good agreement is found for all times $t>t_{H}$ except near the tip for which agreement is achieved only as $t \rightarrow \infty$. This discrepancy is due to the finite time necessary to establish the constant height boundary condition and can be accounted for by using the linear tip solution. Flow for short times $t<t_{H}$ is complicated by the presence of inertia and surface curvature in $z$. However, we show that some of these flows are described by similarity solutions. 


\section{Nomenclature}

The dimensional versions of the dependent and independent parameters used in this study are denoted by a prime symbol (') unless otherwise specified. The use of the subscripted shorthand notation for differentiation should be clear in context.

Dimensionless numbers and quantities

a similarity transform parameter

A cross-sectional flow area

b. $\quad$ similarity transform parameter

$B \quad$ Hoffman shift parameter

$B_{i} \quad$ constant

Bo Bond number, $\Delta \rho g H^{2} / \sigma$

$B o_{H}$. Bond number based on surface curvature, $f \rho g H^{2} / \sigma$

$B o_{H \mathcal{L}}$ Bond number based on curvature and column length, $f \rho g H \mathcal{L} / \sigma$

$C_{i} \quad$ constant

$C a \quad$ Capillary number, $\mu W / \sigma$

$C a_{t}$ time-dependent Capillary number, $\mu H / \sigma t$

$f \quad$ surface curvature function

$F \quad$ generalized similarity function, $F(\eta)$

$\mathcal{F}$ geometric function

$F_{A} \quad$ cross-flow area function

$F_{A_{n}} \quad$ cross-flow area sub-function

$F_{i} \quad$ banded flow resistance function

$h \quad$ meniscus height from $z$-axis, $h(z, t)$

$\mathcal{H}$ mean curvature

$j \quad$ aspect ratio function, $1+D_{1} / D_{2}$

$k$ geometric function, $\phi(1-\phi / 2)$

$K$ numerically determined friction factor coefficient [9]

$\mathcal{L} \quad$ tip location, $\mathcal{L}(t)$

$n \quad$ number of sides on $n$-sided regular polygon

$N_{D} \quad$ index of refraction

Oh Ohnesorge number, $\mu /(\sigma \rho H)^{1 / 2}$

$P$ pressure

$\bar{P}_{o} \quad$ scaled pressure, $P_{o} / \cos ^{2} \Omega$

$\mathcal{P} \quad$ wetted perimeter

$q \quad$ constant volumetric flow rate

$\dot{Q} \quad$ volumetric flow rate, $\dot{Q}(z, t)$

$\mathcal{R} \quad \epsilon^{2} \sin ^{4} \alpha / f O h^{2}$

Re Reynolds number, $W H / \nu$ 


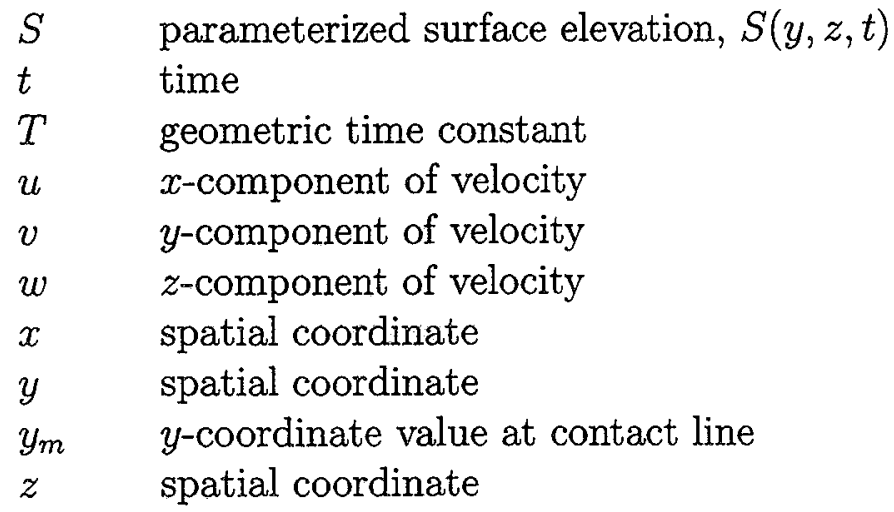

Greek Symbols

$\begin{array}{ll}\alpha & \text { corner half-angle } \\ \beta & \text { numerically determined dimensionless flow resistance [11] } \\ \delta & \text { surface curvature angle, } \pi / 2-\alpha-\theta \\ \epsilon & \text { fluid column slenderness ratio } \\ \gamma & \text { refraction angle } \\ \Gamma & \text { slope function for linear tip solution, } \Gamma(\epsilon) \\ \eta & \text { generalized similarity parameter } \\ \eta_{R R} & \text { surface viscosity [11] } \\ \theta & \text { contact (wetting) angle } \\ \kappa & \text { dimensionless drop volume } \\ \lambda & \text { invariant transform coefficient } \\ \mu & \text { dynamic viscosity } \\ \nu & \text { kinematic viscosity } \\ \xi & \text { wavenumber } \\ \rho & \text { density } \\ \sigma & \text { surface tension } \\ \tau & \text { transformed time }\left(=F_{i} t / 2\right) \\ \phi & \delta / \Omega \\ \Omega & \text { complimentary corner angle, } \pi / 2-\alpha\end{array}$

Dimensional Quantities

c linear fit coefficient-intercept

$D, D_{i}$ face width of test cell

$g \quad$ acceleration field strength, gravity

$g_{i} \quad$ component of $g$ in $i$-direction

$g_{\circ} \quad$ normal gravity, $9.8 \mathrm{~m} / \mathrm{s}^{2}$

$G \quad$ flow coefficient 
$H \quad$ characteristic meniscus height

$L \quad$ characteristic length of fluid column, or cell length

$m \quad$ linear fit coefficient-slope

$R \quad$ radius of curvature

$R_{c} \quad$ local mean radius of curvature

$t_{f} \quad$ curve fit time origin

$t_{o} \quad$ inertial reorientation time, response time 1-g to low-g

$W \quad$ characteristic velocity

$\mathcal{Z}$ global meniscus location on cell centerline

Vectors

$\mathbf{k}$ inward normal to container wall

n outward normal to surface $S$

$t_{1}$ streamwise unit tangent on $S$

$\mathrm{t}_{2}$ cross-flow unit tangent on $S$

$\mathrm{v}$ velocity

Subscripts, Other

$+\quad$ invariant transform quantity

implies dummy variable

\langle\rangle area-averaged quantity

$0,1, \ldots$ counters for order of expanded quantity, constants, etc.

$I \quad$ referring to limit $\alpha^{2} \ll 1$

II refers to limit $\Omega^{2} \ll 1$, free surface condition

$I I_{c} \quad$ refers to limit $\Omega^{2} \ll 1$, captive surface condition

cl contact line quantity

$d \quad$ dynamic quantity

exp experimentally measured quantity

$H \quad$ quantity of constant height location

hyd hydraulic diameter quantity, free surface

$h y d_{c} \quad$ hydraulic diameter quantity, captive surface

$i \quad$ counter

ic inscribed circle quantity

$n$-poly quantity for $n$-sided regular polygonal cross-section

rect quantity for rectangular cross-section

T1 selected test, $2.0 \mathrm{cs}$ PDMS in $22.6 \mathrm{~mm}$ eq. triangular cell

T2 selected test, $10.0 \mathrm{cs}$ PDMS in $12.0 \mathrm{~mm}$ eq. triangular cell

tip quantity evaluated at tip

stat static value 
visc quantity attributed to viscous effect

wall evaluated at wall-corner axis location of vessel 


\section{Contents}

Preliminary Pages

Abstract .......................... i

Nomenclature .............................. ii

List of Tables . . . . . . . . . . . . . . . . . . vi vi

List of Figures . . . . . . . . . . . . . . . vii

1 Introduction 1

1.1 Mechanism and Motivation . . . . . . . . . . . . . . . 1

1.2 Background and Scope of Research . . . . . . . . . . . . . 3

2 Analysis: Governing Equations $\quad 6$

2.1 Analysis of Flow in an Isolated Corner . . . . . . . . . . 7

2.2 Asymptotic Equations . . . . . . . . . . . . . . . 11

2.3 Solutions for $F_{i} \ldots \ldots \ldots \ldots \ldots \ldots$

2.4 Steady Solution . . . . . . . . . . . . . . 25

2.5 Infinite Column Solution . . . . . . . . . . . . . 25

2.6 Similarity Solutions . . . . . . . . . . . . . . . 27

2.6.1 Capillary Rise (Constant Height) . . . . . . . . . 28

2.6.2 Spreading Drop (Constant Volume) . . . . . . . . . . 29

2.6 .3 Constant Flow . . . . . . . . . . . . . . 30

2.6.4 Generalized Similarity Solution . . . . . . . . . . . . 32 
2.6.5 Inclusion of Gravity in $x$-direction . . . . . . . . . . . 33

$2.6 .6 z$-Curvature Consideration . . . . . . . . . . 34

2.6.7 Summary of Closed Form Results (dimensional) . . . . . . . 36

2.6.8 Determination of $H$, Capillary Rise . . . . . . . . 38

2.7 Quantified Constraints . . . . . . . . . . . . . . . 39

2.7.1 Assumptions for Capillary Rise . . . . . . . . . . . 40

2.7.2 Assumptions for Spreading Drop . . . . . . . . . . . 42

2.7.3 Assumptions for Constant Flow . . . . . . . . . . . 44

2.7.4 Abbreviated Summary of Assumptions . . . . . . . . . . 45

3 Capillary Rise Experiments $\quad 47$

3.1 Introduction to Low-g Experimentation . . . . . . . . . . . . . 48

3.2 Description of the Experiment f . . . . . . . . . . 48

3.3 Preliminary Measurements . . . . . . . . . . . . . . 51

3.3 .1 Tip Location, $\mathcal{L}(t) \ldots \ldots \ldots 52$

3.3 .2 Global Meniscus Height, $\mathcal{Z}(t) \ldots \ldots \ldots 5$

3.4 Detailed Measurements . . . . . . . . . . . . . 55

3.4.1 Surface Profile, $h(z, t)$ for data set T1 . . . . . . . . 57

3.4 .2 Surface Profile, $h(z, t)$ for data set T2 . . . . . . . 61

3.5 Discussion . . . . . . . . . . . . . . . 63

3.5.1 Tip Solution Approximation . . . . . . . . . . 68

3.5.2 Start-up Problem . . . . . . . . . . . . . . 68

3.5.3 Further Remarks ................ 71

4 Conclusion $\quad 72$

4.1 Summary of Results . . . . . . . . . . . . . . 72

4.1.1 Analytical Contributions . . . . . . . . . . 72

4.1.2 Experimental Contributions . . . . . . . . . . 74

4.2 Recommendations for Future Work . . . . . . . . . . . . 75

vii 
Bibliography

$\begin{array}{lr}\text { Appendices } & 80\end{array}$

$\begin{array}{lr}\text { A Analytical Details } & \mathbf{8 0}\end{array}$

A.1 Equations and Boundary Conditions ... . . . . . . . . . 80

A.2 $F_{i}$ Details ........................ 81

A.3 Similarity Details . . . . . . . . . . . . . . . . . 84

A.4 Similarity with $g_{x} \ldots \ldots \ldots \ldots$. . . . . . . . . . 85

$\begin{array}{ll}\text { B Correction for Optical Distortion } & 87\end{array}$ 


\section{List of Tables}

2.1 Nondimensionalized dependent and independent variables. . . . . . 8

2.2 Values of $\beta, F_{i}$, and $F_{\text {hyd }} \ldots \ldots \ldots \ldots \ldots \ldots \ldots$

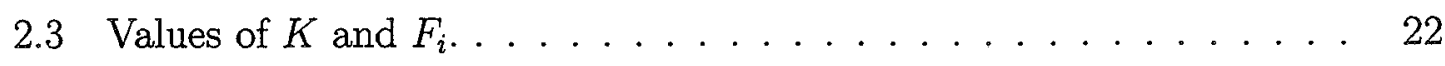

2.4 Summarized values from the similarity solutions. . . . . . . . . . 31

2.5 Selected values from the generalized similarity equation. . . . . . . 32

2.6 Summary of constraints. . . . . . . . . . . . . . 46

3.1 Test fluids and properties. . . . . . . . . . . . . . 50

3.2 Test cell data for capillary rise tests. . . . . . . . . . . . . 50

3.3 Time constants for $\mathrm{T} 1$ and $\mathrm{T} 2$ for capillary rise. . . . . . . . . . . 68

3.4 Time constants for $\mathrm{T} 1$ and $\mathrm{T} 2$ for start-up problem. . . . . . . . 71 


\section{List of Figures}

1.1 Partially filled square container. . . . . . . . . . . . . 2

1.2 Capillary rise due to a step reduction in $g \ldots \ldots \ldots \ldots$

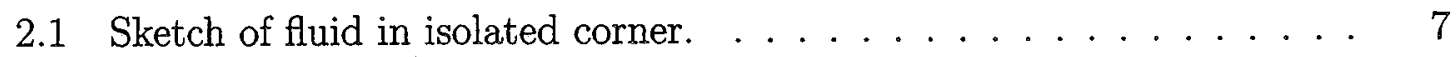

2.2 Sketch to show variables used in the cross-flow formulation. . . . . . 13

2.3 The parameters $f$ and $F_{A}$ as a function of $\delta$ for various $\alpha . \ldots \ldots l$

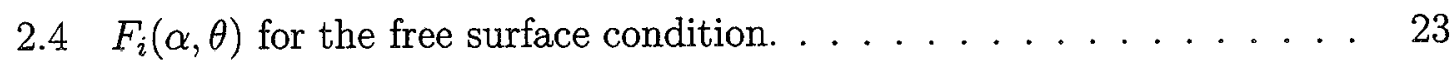

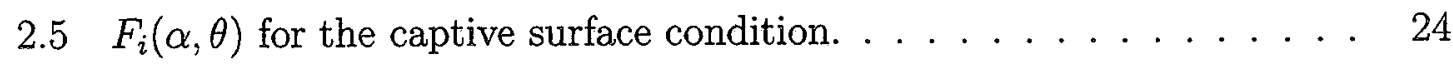

2.6 Geometric time constant $T(\alpha, \delta) \ldots \ldots \ldots \ldots \ldots \ldots$

2.7 Similarity function $\mathrm{F}^{+}\left(\eta^{+}\right)$for capillary rise, spreading drop, and constant flow. . . . . . . . . . . . . . . . . . 31

2.8 Similarity function $F^{+}\left(\eta^{+}\right)$for $a \geq-1 / 3 \ldots \ldots \ldots \ldots \ldots$

2.9 Similarity function $F(\eta)$ for capillary rise with gravity, $g_{x} \ldots \ldots \ldots 35$

3.1 Selected frames from cinefilm records of capillary rise in equilateral triangles. . . . . . . . . . . . . . . . . . . . 49

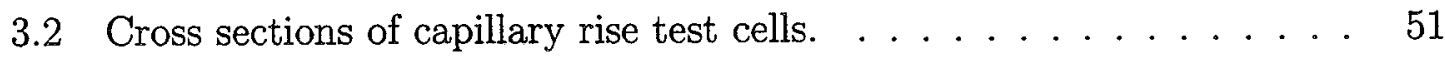

$3.3 \mathcal{L}$ vs. $t^{1 / 2}$ in acrylic triangle: Effect of viscosity. . . . . . 53

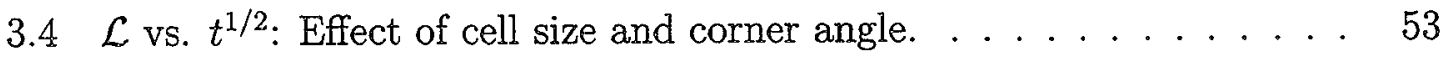

$3.5 \mathcal{L}$ vs. $t^{1 / 2}$ in acrylic rectangular cells: Effect of aspect ratio. . . . . 54

$3.6 \mathcal{Z}$ vs. $t^{1 / 2}$ in acrylic triangle: Effect of viscosity. . . . . . . 55

$3.7 \mathcal{Z}$ vs. $t^{1 / 2}$ in acrylic cells: Effect of cell shape and size. . . . . . . 56

$3.8 \mathcal{L}$ vs. $t$ in acrylic triangular cells with $c+m\left(t-t_{f}\right)^{1 / 2}$ fits. $\ldots \ldots \quad 57$ 
$3.9 \mathcal{Z}$ vs. $t$ for $\mathrm{T} 1$ and $\mathrm{T} 2 \ldots \ldots \ldots \ldots \ldots \ldots \ldots$

3.10 Traces of the surface height $h(z, t)$ for $\mathrm{T} 1 \ldots \ldots \ldots \ldots$

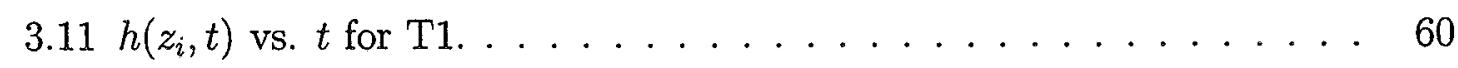

$3.12 h\left(z_{i}, t\right)$ vs. $t$ for $\mathrm{T} 1, t>0.7 \mathrm{~s} . \ldots \ldots \ldots \ldots \ldots \ldots \ldots \ldots \ldots \ldots$

$3.13 h$ vs. $z / t^{1 / 2}$ and $z /\left(t-t_{H}\right)^{1 / 2}$ for several traces. Data of T1. . . . 62

$3.14 h$ vs. $z /\left(t-t_{o}\right)^{1 / 2}$ for $\mathrm{T} 1$ with $t_{o}=0.09 \mathrm{~s} \ldots \ldots \ldots \ldots \ldots$

3.15 Traces of surface height $h(z, t)$ for $\mathrm{T} 2 \ldots \ldots \ldots \ldots \ldots$

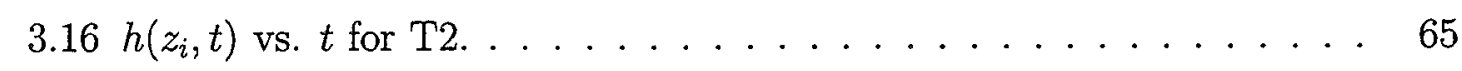

$3.17 h$ vs. $z / t^{1 / 2}$ and $z /\left(t-t_{H}\right)^{1 / 2}$ for several traces. Data of T2. . . . 66

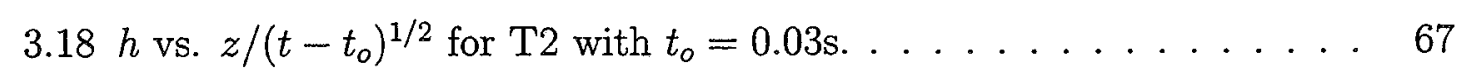

$3.19 h / t^{1 / 5}$ vs. $z / t^{3 / 5}$ for $\mathrm{T} 2 . \ldots \ldots \ldots \ldots \ldots \ldots$

$3.20 h\left(z_{i}, t\right)$ as a function of $t$ for $\mathrm{T} 2, z_{i} \simeq z_{H} \ldots \ldots \ldots \ldots$

B.1 Schematic of ray trace correction for $h(z, t)$ measurements. . . . 88 


\section{Chapter 1}

\section{Introduction}

We commonly think of capillary phenomena as relevant to small systems such as capillary tubes, candle wicks, paper towels, etc. This is because on earth only small systems allow surface tension and wetting phenomena to play a dominant role over the otherwise controlling influence of gravity. In the presence of gravity the degree to which capillary forces can be expected to be significant is measured by the Bond number $B o=\Delta \rho g H^{2} / \sigma$, where $\Delta \rho$ is the density difference across the fluid interface, $g$ is the acceleration of gravity, $H$ is a characteristic length scale (i.e. tube radius, pore size, etc.), and $\sigma$ is the interfacial, or surface, tension. The Bond number is a ratio of gravitational forces to those of surface tension, and low values, $B o \ll 1$, imply capillary dominated systems. The properties $\Delta \rho$ and $\sigma$ are essentially fixed for a given system. For earth bound systems $g$ is also fixed and capillary dominated systems arise only if geometries are small. However, for the low-gravity environment of space, surface tension can dominate fluid systems of large extent.

\subsection{Mechanism and Motivation}

The subject of capillary driven flows in containers with interior corners is perhaps best introduced via an illustrative example. Figure 1.1 depicts a container with a square cross-section partially filled with a liquid in a gravitational field. The majority of the interface is flat owing to the large Bond number of the system. However, as is commonly observed, especially in the corner regions of the container, the interface curves in order to satisfy the contact angle condition along the perimeter of the interface.

A magnified view of the corner region shows that the local radius of curvature 


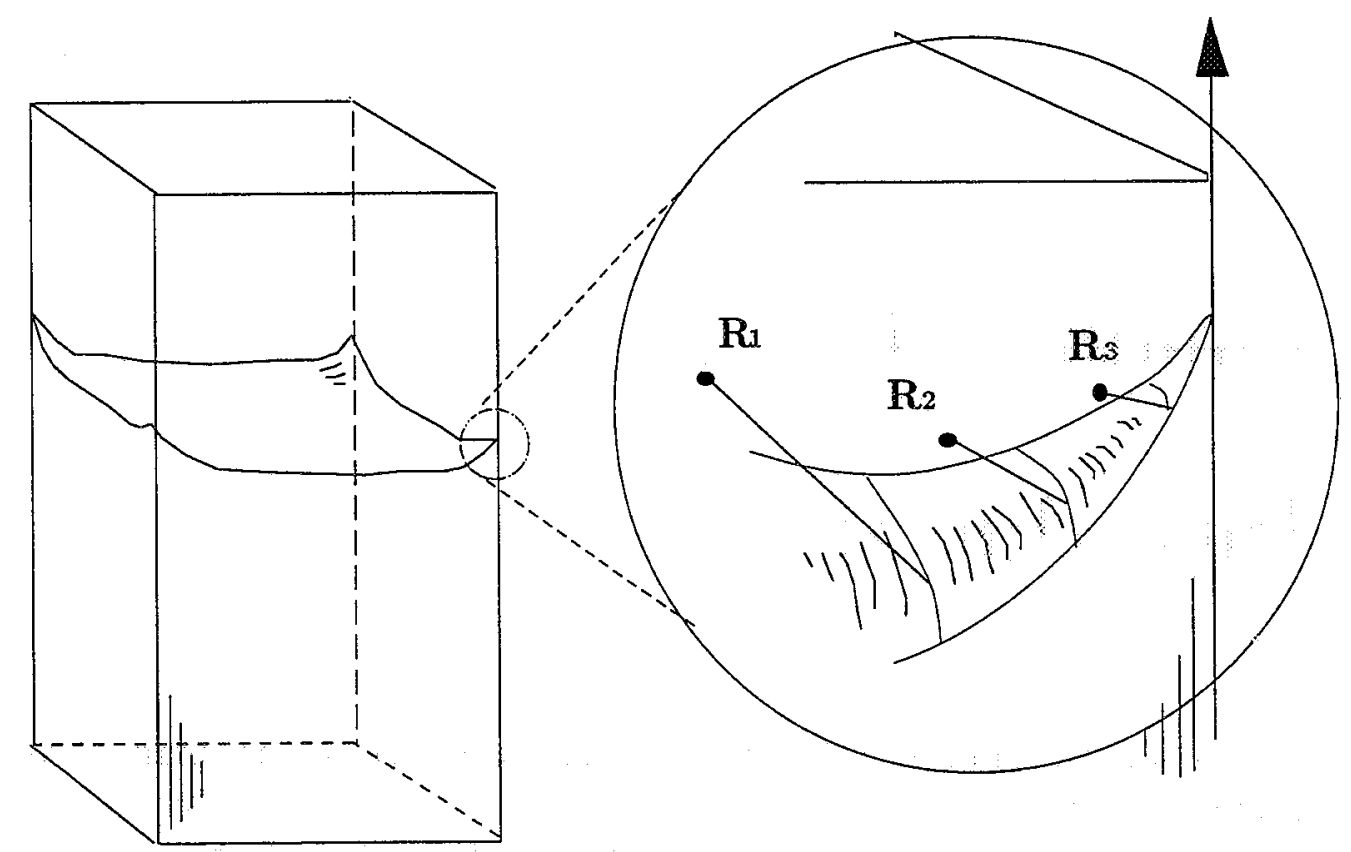

Figure 1.1: Partially filled square container.

$R_{i}$ of the meniscus decreases as the corner is approached. Since the pressure drop across the meniscus is inversely proportional to $R_{i}$ it follows that the pressure in the liquid also decreases as the tip of the meniscus in the corner is approached. Thus, a pressure gradient along the corner is established in the wetting liquid due to increasing interface curvature. This gradient is in turn balanced by the hydrostatic pressure gradient. If gravity were suddenly "turned off," the interface curvature pressure gradient would no longer be balanced and a capillary driven flow along the corners would ensue. The extent to which the fluid rises in the corner during the flow process is dependent on the contact angle $(\theta)$ and corner half-angle $(\alpha)$ of the system. In many cases the fluid will climb to a great height if allowed, provided that the contact angle is low enough. The criterion for when such large scale flows must occur is thoroughly addressed by Concus and Finn [1]-[3] who show that capillary driven flows in corners of infinite extent proceed to infinite distances when the condition

$$
\theta<\pi / 2-\alpha
$$

is satisfied, hereafter referred to as the Concus-Finn condition. ${ }^{1}$ This condition is satisfied in the case of Figure 1.2 which illustrates this phenomenon for a square

\footnotetext{
${ }^{1}$ In their mathematical study, Concus and Finn were the first to obtain the result of the discontinuous behavior of an interface in a corner at the critical value $\theta=\pi / 2-\alpha$. The condition of eq 1.1 appears also in other heuristic studies concerning interfacial phenomena.
} 


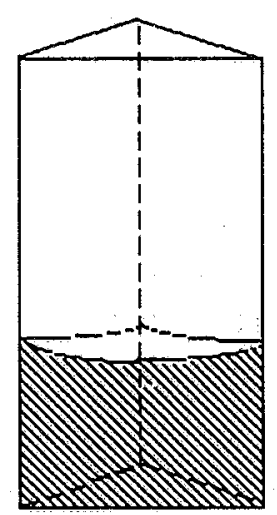

$t=0$

$g=g_{0}$

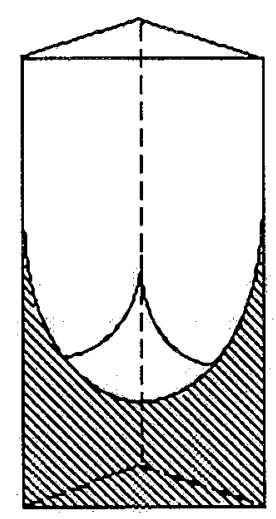

$\mathbf{t}=\mathbf{T 1}$

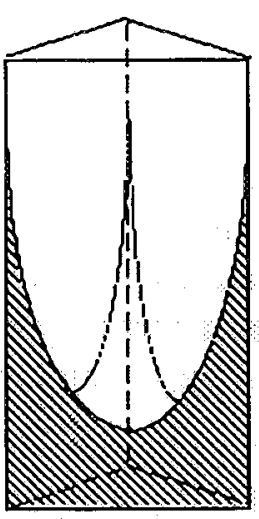

$\mathbf{t}=\mathbf{T 2}$

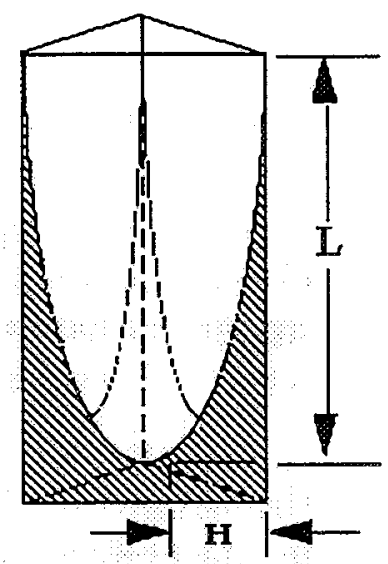

$\mathrm{t}=\mathrm{T} 3$

Low $g$

Figure 1.2: Capillary rise due to a step reduction in $g$.

cross-sectioned container employed in a simple drop tower experiment. (Note that the figure displays only half of the vessel which is bisected along the diagonal.) After release of the container in the drop tower, slender columns of fluid rise in the corners due to capillary forces and act to drain the container by redistributing the fluid along the corners.

Capillary pumping of this general sort arises in numerous contexts and is particularly prevalent in low gravity environments where system designs are heavily impacted by the presence of container irregularities such as corners. Examples of such systems involve most, if not all, in-space fluids management processes, from the positioning, control, and transport of liquids, such as fuels in storage tanks, to thermal systems such as heat pipes and capillary pumped loops, to the storage and handling of biological fluids and wastes. Examples of terrestrial applications include flows in porous media and the wetting and spreading of fluids on irregular surfaces-flow processes which are commonplace in nature and industry.

\subsection{Background and Scope of Research}

A number of investigations have produced useful results on interface configurations (statics), flows, and stability for the capillary behavior of liquids in containers with 
interior corners. ${ }^{2}$ The case of capillary statics has been well addressed by the literature. Included in these studies, for example, are the effects of contact angle and corner half-angle [1] [2], container aspect ratio and corner rounding [4], differing contact angles on adjacent faces of the corner [5], irregular triangular sections [6], and a novel solution approach based on the inclusion of molecular forces [7]. The works of Concus and Finn, who have obtained specific results for a host of cylinders of general section, in part summarized in [8], are exemplary and a review of their work may serve well the designer requiring insight into low-gravity interface shapes.

A significant amount of attention has also been devoted to dynamics. Though the majority of work [9]-[17] has centered on forced flows and/or wicking (imbibition) in "micro-sized" geometries for earth-based applications, low-gravity experiments by Masica (discussed in [1] and [8]) and by Langbein [18], in addition to a multitude of applied space systems studies [19][20] have clearly demonstrated the large-scale nature of such flows in the absence of gravity. Nonetheless, an ample collection of quantitative analytical results on related flows applicable to the unusual conditions of low-gravity, is yet to be acquired. Questions of practical importance concerning the general response time, flow rate, surface profile, and velocity distribution as functions of the container geometry and fluid properties-including the contact angle-remain to be fully resolved for the unique range of system parameters accessible with the reduction of gravity.

This work reports an analytical and experimental investigation to answer these questions for a limited variety of container types and flow scenarios. The analytic portion of the work begins with a description of the simplified problem of capillary-driven flow along an isolated corner and follows with an asymptotic analysis employing the lubrication approximation. The velocity scale used in the nondimensionalization of the equations correctly captures the geometric effects of corner angle and contact angle and reduces a numerically determined geometric function, used by previous investigators, $\sim O(1)$ to $\infty$, to one which is tightly banded and $\sim O(1)$. The governing partial differential equation is first solved by a regular perturbation method for the case of the infinite corner. The equation is then transformed yielding similarity solutions for the cases of capillary rise, a spreading drop, and a constant flow rate condition. The impact of gravity, three dimensional surface curvature, inertia, and the moving contact line boundary condition are considered.

A description of the experimental techniques, selection of test results, and discussion thereof follows for the special case of capillary rise. It is shown that the analytical results, which often yield closed form expressions, are well-suited for use in describing such flows, even at small times. Applications may be made to problems

\footnotetext{
2Interior corners are often referred to as "grooves," "edges," or "wedges" in the literature.
} 
arising in the low-gravity environment. Only systems which satisfy the Concus-Finn condition are considered in detail. For an analysis of the case $\theta>\pi / 2-\alpha$ see Langbein [21] who determines both the shape and stability of fluid columns in corners of infinite extent. 


\section{Chapter 2}

\section{Analysis: Governing Equations}

The particular corner flow problem illustrated in Figure 1.2 was recently studied by Dong and Chatzis [17] who employ $0.3-0.5 \mathrm{~mm}$ diameter tubes of square cross-section in an analysis and experiment on imbibition as relates to flows in porous media. As in previous and related studies [11][12], it is sufficient for the authors to simply state the assumptions (locally parallel flow, negligible corner axis curvature and inertia, etc.) and proceed with solutions to the reduced set of governing equations. The 'small' container size $\lesssim O(1 \mathrm{~mm})$ of these experiments provides a firm basis for such an approach. For a system of equivalent Bond number in a low-gravity environment, however, the assumptions come into question. For example, the Ohnesorge number,

$$
O h=\frac{\mu}{(\sigma \rho H)^{1 / 2}}
$$

where $\mu$ is the dynamic viscosity of the liquid, or rather $1 / O h^{2}$, is the correct choice as a measure of the influence of inertia in capillary driven flows; similarly, as is the Reynolds number in forced flows. For ground-based studies $\left(g=1 g_{0}\right)$, a strong capillary presence is maintained when the characteristic dimension $H$ scales as the capillary length, $H \sim(\sigma / \rho g)^{1 / 2}$. Therefore,

$$
\frac{1}{O h^{2}} \sim \frac{\sigma \rho}{\mu^{2}}\left(\frac{\sigma}{\rho g}\right)^{1 / 2}
$$

Thus, in a microgravity environment $\left(g=10^{-6} g_{o}\right)$ the parametric range of $1 / O h^{2}$ is extended up to 3 orders of magnitude. More dramatic yet are the changes which might be expected in the viscous time scale $t_{v i s c} \sim \rho H^{2} / \mu$. With a 1000-fold increase of $H$, realizable in a low-gravity environment, $t_{v i s c}$ increases by $10^{6}$ over its normal gravity counterpart! To accommodate for such possible scenarios an effort here is 


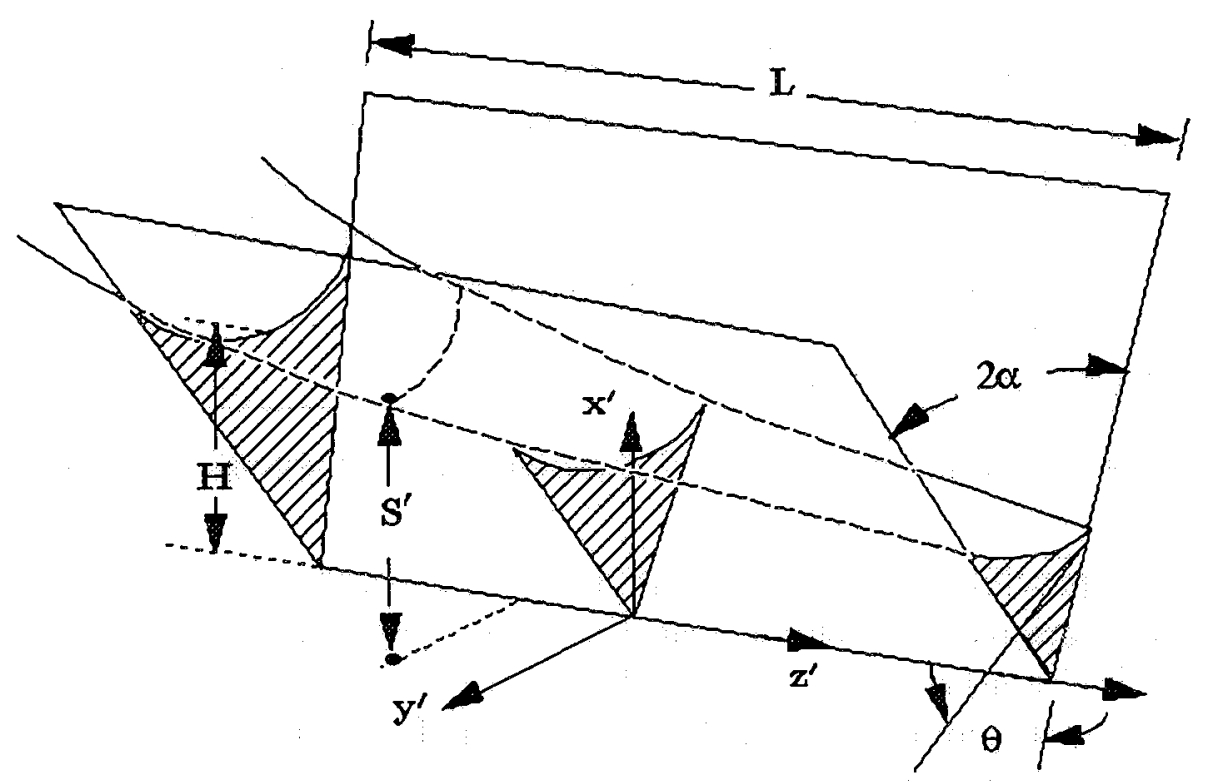

Figure 2.1: Sketch of fluid in isolated corner.

made towards generality. The governing equations are scaled in such a manner that the application of asymptotic techniques leads to simply expressed solutions for a variety of corner flow problems. A natural means for assessing the validity of the solutions arises and the many constraints of the problems are quantified.

\subsection{Analysis of Flow in an Isolated Corner}

Since the corner flow controls much of the flow throughout the container, it is natural to analyze the fluid in this region first. An isolated interior corner of angle $2 \alpha$ is depicted in Figure 2.1. The corner is partially filled with a fluid satisfying the Concus-Finn condition. The coordinate axes are labeled on the figure, the $z^{\prime}$-axis corresponding to the corner axis, and $S^{\prime}$ is the parameterized height of the free surface as measured from the $y^{\prime}-z^{\prime}$ plane.

\section{Nondimensionalization}

As shown in Figure 2.1, $L$ is the characteristic length of the column of fluid along the $z^{\prime}$-axis and $H$ is the characteristic height of the meniscus in the corner along the $x^{\prime}$-axis. The ratio of these length scales $(\epsilon=H / L)$ is crucial to the analysis to follow. 


\begin{tabular}{|l|l|l|}
\hline Lengths & Velocities & Other \\
\hline$x=x^{\prime} / H$ & $u=u^{\prime} / \epsilon W$ & $P=H f P^{\prime} / \sigma$ \\
$y=y^{\prime} / H \tan \alpha$ & $v=v^{\prime} / \epsilon W \tan \alpha$ & $t=W t^{\prime} / L$ \\
$z=z^{\prime} / L$ & $w=w^{\prime} / W$ & $A=A^{\prime} / H^{2} \tan \alpha$ \\
$S=S^{\prime} / H$ & $\langle w\rangle=\langle w\rangle^{\prime} / W$ & $\dot{Q}=\dot{Q}^{\prime} / W H^{2} \tan \alpha$ \\
$h=h^{\prime} / H$ & $W=\epsilon \sigma \sin ^{2} \alpha / \mu f$ & \\
$\mathcal{L}=\mathcal{L}^{\prime} / L$ & & \\
$y_{m}=y_{\text {max }}^{\prime} / H \tan \alpha$ & & \\
\hline
\end{tabular}

Table 2.1: Nondimensionalized čependent and independent variables.

Table 2.1 provides the quantities used to nondimensionalize the governing equations. Primes are used to denote dimensional dependent and independent variables. The length scales arise naturally from the geometry of the problem. The pressure is scaled using $\sigma / H f$, and $f=f(\alpha, \theta)$ is a geometric function describing the curvature of the meniscus in the $x^{\prime}-y^{\prime}$ plane, to be defined later. For this curvature scale to be valid the curvature in the $x^{\prime}-z^{\prime}$ plane $\left(\sim \partial^{2} S^{\prime} / \partial z^{2}\right)$ must be small by comparison. In other words, the constraint of $\epsilon^{2} f \ll 1$ must be imposed. The characteristic $z^{\prime}-$ component of velocity, $W$, is determined through a balance of pressure and viscous forces and uniquely incorporates the geometric influence of $\alpha$ on the solution domain. The scales for $u^{\prime}$ and $v^{\prime}$ are determined via continuity. A passive overlaying fluid is assumed and, for the time being, body forces are ignored.

On substitution of the Table 2.1 quantities, the individual component equations of the nondimensionalized Navier-Stokes equation become

$$
\begin{aligned}
\epsilon^{2} \mathcal{R} \frac{D u}{D t} & =-P_{x}+\epsilon^{2} \nabla^{2} u \\
\epsilon^{2} \mathcal{R} \tan ^{2} \alpha \frac{D v}{D t} & =-P_{y}+\epsilon^{2} \tan ^{2} \alpha \nabla^{2} v \\
\mathcal{R} \frac{D w}{D t} & =-P_{z}+\nabla^{2} w
\end{aligned}
$$

with

$$
\begin{gathered}
\frac{D}{D t}=\frac{\partial}{\partial t}+u \frac{\partial}{\partial x}+v \frac{\partial}{\partial y}+w \frac{\partial}{\partial z} \\
\nabla^{2}=\sin ^{2} \alpha \frac{\partial^{2}}{\partial x^{2}}+\cos ^{2} \alpha \frac{\partial^{2}}{\partial y^{2}}+\epsilon^{2} \sin ^{2} \alpha \frac{\partial^{2}}{\partial z^{2}}
\end{gathered}
$$

and

$$
\mathcal{R}=\frac{\epsilon^{2}}{f O h^{2}} \sin ^{4} \alpha
$$


$O h$ is the Ohnesorge number given by $O h=(C a / R e)^{1 / 2}=\mu /(\rho \sigma H)^{1 / 2}$, where $C a=$ $\mu W / \sigma$ and $R e=\rho W H / \mu$ are the Capillary and Reynolds numbers, respectively. Defined as such, $O h$ serves as a ratio of the restoring-force time scale of surface tension $\left(\sim\left(\rho H^{3} / \sigma\right)^{1 / 2}\right)$ to the viscous time scale $\left(\sim H^{2} / \nu\right)$. For $O h^{2} \ll 1$ systems are underdamped to 'abrupt' disturbances while for $O h^{2} \gg 1$ viscous forces dominate. The accessible parametric range of $\mathcal{R}$ is increased several orders of magnitude when gravity is low. This influence is felt through $O h$ as described earlier. In general, the condition $O h^{2} \gg 1$ is not often satisfied even for 'small' normal-gravity systems.

The continuity equation is

$$
\nabla \cdot \mathbf{v}=0
$$

The boundary conditions for the above system are as follows: The no-slip condition is

$$
\mathbf{v}=0
$$

and applies along the walls. The scaled $(\times H / \mu W)$ streamwise and transverse zero shear stress conditions on $S$ are

$$
\begin{gathered}
\frac{\epsilon k_{1} k_{2}^{2}}{\tan \alpha}\left[\left(1-S_{y}^{2} \cot ^{2} \alpha+\epsilon^{2} S_{z}^{2}\right)\left(u_{y}+\right) v_{x} \tan ^{2} \alpha-S_{z}\left(w_{y}+\epsilon^{2} v_{z} \tan ^{2} \alpha\right)\right) \\
\left.+2 S_{y}\left(u_{x}-v_{y}-S_{z}\left(w_{x}+\epsilon^{2} u_{z}\right)-\epsilon^{2} S_{z}^{2}\left(w_{z}+v_{y}\right)\right)\right]=0
\end{gathered}
$$

and

$$
\begin{gathered}
k_{1} k_{2}\left[\left(1-\epsilon^{2} S_{z}^{2}\right)\left(w_{x}+\epsilon^{2} u_{z}\right)-S_{y}\left(w_{y} \cot ^{2} \alpha+\epsilon^{2} v_{z}\right)+2 \epsilon^{2} S_{z}\left(u_{x}-w_{z}\right)\right. \\
\left.-\epsilon^{2} S_{y} S_{z}\left(u_{y} \cot ^{2} \alpha+v_{x}\right)\right]=0
\end{gathered}
$$

respectively, where

$$
\begin{aligned}
& k_{1}=\left(1+\epsilon^{2} S_{z}^{2}\right)^{-1 / 2} \\
& k_{2}=\left(1+|\nabla S|^{2}\right)^{-1 / 2}
\end{aligned}
$$

and

$$
|\nabla S|^{2}=S_{y}^{2} \cot ^{2} \alpha+\epsilon^{2} S_{z}^{2}
$$

The normal stress condition on $S$ is

$$
-P+2 \epsilon^{2} k_{2}^{2}[M] \sin ^{2} \alpha=2 \mathcal{H}
$$

where

$$
[M]=u_{x}-S_{y} \cot ^{2} \alpha\left(u_{y}+v_{x} \tan ^{2} \alpha\right)-S_{z}\left(w_{x}+\epsilon^{2} u_{z}\right)+S_{y} S_{z} \cot ^{2} \alpha\left(w_{y}+\epsilon^{2} v_{z} \tan ^{2} \alpha\right)
$$




$$
+S_{y}^{2} v_{y} \cot ^{2} \alpha+\epsilon^{2} S_{z}^{2} w_{z}
$$

and $2 \mathcal{H}$ is twice the mean curvature of the interface given by

$$
2 \mathcal{H}=f k_{2}^{3} \cot ^{2} \alpha\left[S_{y y}\left(1+\epsilon^{2} S_{z}^{2}\right)+\epsilon^{2} S_{z z}\left(\tan ^{2} \alpha+S_{y}^{2}\right)-2 \epsilon^{2} S_{y} S_{z} S_{y z}\right]
$$

Two (of four) boundary conditions on $S$ are the meniscus centerline location and symmetry conditions on $y=0$; namely

$$
\begin{array}{lll}
S=h & \text { on } & y=0 \\
\frac{\partial S}{\partial y}=0 & \text { on } & y=0
\end{array}
$$

where $h=h(z, t)$. The remaining two boundary conditions concern the treatment of the moving contact line. This poses particular difficulties in that the physics of the moving contact line is not fully understood due to a well known stress singularity at the contact line itself [22][23].

The simplest conceivable formulation of the moving contact line boundary condition is the case of fluid statics where the condition

$$
\mathbf{n} \cdot \mathbf{k}=\cos \theta
$$

is applied. $\theta$ is the static (or perhaps equilibrium) contact angle of the fluid-solid pair, ${ }^{1} \mathbf{k}$ is the inward normal to the container walls (on $y= \pm x$ ) given by $\mathbf{k}=$ $(\sin \alpha, \mp \cos \alpha, 0)$, and $\mathbf{n}$ is the outward unit normal to $S$,

$$
\mathrm{n}=\left(1+|\nabla S|^{2}\right)^{-1 / 2}\left(1,-S_{y} \cot \alpha,-\epsilon S_{z}\right)
$$

This boundary condition at the contact line is strictly valid when $\mathbf{v}=0$ and may be approximately correct when the fluid velocity perpendicular to the contact line is small. This is, in general, the case for the corner flow problem discussed here where the predominant flow direction is parallel to the contact line. The use of eq 2.19 will be discussed in subsequent sections. The net effect of its application is that the two additional boundary conditions for $S$, namely, the slope and the location of the interface at the contact line, may be determined in terms of $h$. These conditions are given by

$$
\frac{1+S_{y} \cot ^{2} \alpha}{\left(1+|\nabla S|^{2}\right)^{1 / 2}}=\frac{\cos \theta}{\sin \alpha} \quad \text { on } \quad y=y_{m}
$$

\footnotetext{
${ }^{1}$ If contact angle hysteresis is present and the contact line is in motion, $\theta$ might best be taken as the advancing angle on first approximation. For a clear definition of the distinction between static and equilibrium contact angle and for a discussion of the difficulties faced when contact angle hysteresis is present in systems exhibiting partial wetting see [23, p. 328].
} 
and

$$
S=y_{m} \quad \text { on } \quad y=y_{m}
$$

respectively, the latter condition being the interface/wall intersection condition and $y_{m}$ being the value of $y$ at the contact line.

A mass balance in the $z$-direction gives

$$
\frac{\partial A}{\partial t}=-\frac{\partial \dot{Q}}{\partial z}=-\frac{\partial}{\partial z} A\langle w\rangle
$$

where $A$ is the cross-sectional area of the flow in the $x-y$ plane and $\dot{Q}$ is the volumetric flow rate in the $z$-direction, namely

$$
\begin{aligned}
& A=2 \int_{0}^{y_{m}}(S-y) d y \\
& \dot{Q}=2 \int_{0}^{y_{m}} \int_{y}^{S} w d x d y
\end{aligned}
$$

and $\langle w\rangle \equiv \dot{Q} / A$. The inlet and outlet conditions at $z=0$ and $z=1$ and the initial conditions remain to be addressed.

\section{$2.2 \quad$ Asymptotic Equations}

Though eqs 2.2-2.4 and their associated boundary conditions eqs 2.9-2.22 are a formidable set of equations, the slenderness ratio $\epsilon$, appears as an obvious choice for a small parameter upon which to base an asymptotic analysis. The limit $\epsilon^{2} \ll 1$ is the common lubrication approximation and, as in many other contexts [24], is motivated by observations of the flow phenomena such as that depicted in Figure 1.2 .

For the case $\epsilon^{2} \ll 1$ the dependent variables of the problem may be expanded in asymptotic series

$$
\begin{aligned}
& u=u_{o}+\epsilon^{2} u_{1}+\ldots \\
& v=v_{\circ}+\epsilon^{2} v_{1}+\ldots \\
& w=w_{o}+\epsilon^{2} w_{1}+\ldots \\
& P=P_{o}+\epsilon^{2} P_{1}+\ldots \\
& S=S_{o}+\epsilon^{2} S_{1}+\ldots
\end{aligned}
$$


where terms of $O(\epsilon)$ are absorbed into the leading order terms for convenience. In the limit $\epsilon^{2} \ll 1$, assuming for the present $\mathcal{R} \sim O\left(\epsilon^{2}\right)$, the leading order equations reduce to the $z$-component momentum equation

$$
\frac{\partial P_{o}}{\partial z}=\sin ^{2} \alpha \frac{\partial^{2} w_{o}}{\partial x^{2}}+\cos ^{2} \alpha \frac{\partial^{2} w_{o}}{\partial y^{2}}
$$

Also to leading order, the boundary conditions for eq 2.29 , eqs $2.9,2.11$, and the constraint of flow symmetry about $y=0$ may be expressed as

$$
\begin{gathered}
w_{o}=0 \quad \text { on } y=x \\
\frac{\partial w_{o}}{\partial x}-\frac{\partial S_{o}}{\partial y} \frac{\partial w_{o}}{\partial y} \cot ^{2} \alpha=0 \quad \text { on } x=S_{\circ} \\
\frac{\partial w_{o}}{\partial y}=0 \quad \text { on } y=0
\end{gathered}
$$

respectively. The normal stress condition can now be written as

$$
-P_{o}=f \cot ^{2} \alpha \frac{\partial^{2} S_{o}}{\partial y^{2}}\left(1+\left(\frac{\partial S_{o}}{\partial y}\right)^{2} \cot ^{2} \alpha\right)^{-3 / 2}
$$

with the leading order boundary conditions

$$
\begin{gathered}
S_{o}=h \quad \text { on } y=0 \\
\frac{\partial S_{o}}{\partial y}=0 \quad \text { on } y=0 \\
S_{o}=y_{m} \quad \text { on } y=y_{m} \\
\frac{1+\frac{\partial S_{o}}{\partial y} \cot ^{2} \alpha}{\left(1+\left(\frac{\partial S_{o}}{\partial y}\right)^{2} \cot ^{2} \alpha\right)^{1 / 2}}=\frac{\cos \theta}{\sin \alpha} \quad \text { on } y=y_{m}
\end{gathered}
$$

Noting that $P_{o}=P_{o}(z, t)$, the normal-stress equation may be solved directly with its associated boundary conditions to find $S_{o}, y_{m}, f$, and $P_{o}(h)$. This procedure results in forms of these quantities which are unnecessarily awkward. Less cumbersome forms are obtained by solving the geometrical 'cross-flow' problem sketched in Figure 2.2 for the pressure in the liquid as a function of meniscus height $h$, with the dimensional radius of curvature ${ }^{2}$ taken to be constant $R^{\prime}=f h^{\prime}$. This yields

$$
P_{\circ}=-\frac{1}{h}
$$

\footnotetext{
${ }^{2}$ Note that the curvature of the interface is not a constant in the dimensionless $x-y$ plane.
} 


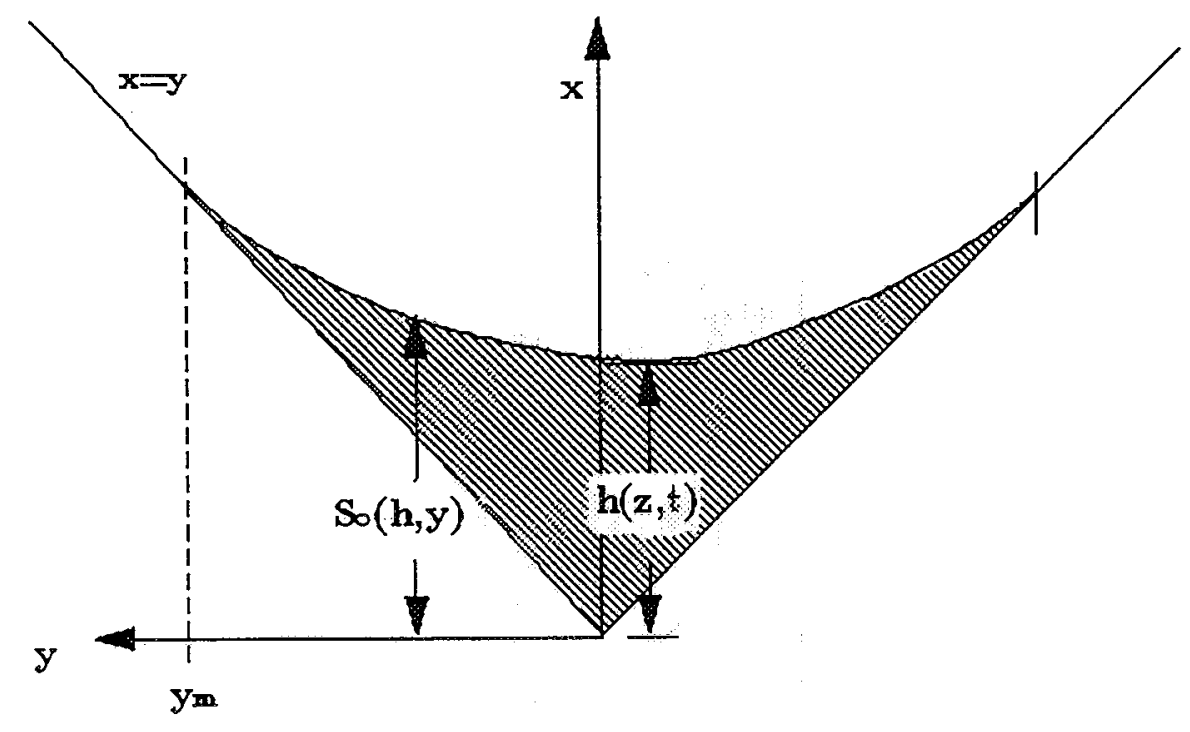

Figure 2.2: Sketch to show variables used in the cross-flow formulation, eqs 231-234. The height of the interface at a given $z$ is $S_{o}(h, y)$, where $h(z, t)$ is the height at $y=0$.

$$
\begin{gathered}
S_{o}=h(1+f)-f h\left(1-\left(\frac{y \tan \alpha}{f h}\right)^{2}\right)^{1 / 2} \\
y_{m}=\frac{f h \sin \delta}{\tan \alpha} \\
f=\left(\frac{\cos \theta}{\sin \alpha}-1\right)^{-1}=\left(\frac{\sin (\alpha+\delta)}{\sin \alpha}-1\right)^{-1}
\end{gathered}
$$

where $\delta \equiv \pi / 2-\alpha-\theta$. The parameter $\delta$ is introduced as a preferred angle (as opposed to $\theta$ ) which measures the degree of curvature of the interface in the $x-y$ plane. The condition $0<\delta<\pi / 2$ is the Concus-Finn condition. ${ }^{3}$ The use of $f$ in the nondimensionalization of the problem leads to the unique expression for $P_{o}$ given in eq 2.31 and the sign of $f$ indicates the anticipated flow direction of the $z$-component velocity scale $W$. The parameter $f$ characterizes the strength of the driving force for the flow due to curvature of the interface, independent of the cross-flow area. The parameter $F_{A}=F_{A}(\alpha, \delta)$ is the cross-sectional area function $\left(A^{\prime}=h^{\prime 2} F_{A}\right)$ and may

\footnotetext{
${ }^{3}$ The case of $\delta<0$ is also accounted for in this result. Note that $f$ can change sign depending on the value of $\delta$ indicating a change in positive to negative curvature of the interface. Positive curvature is depicted in Fig. 2.2 .
} 


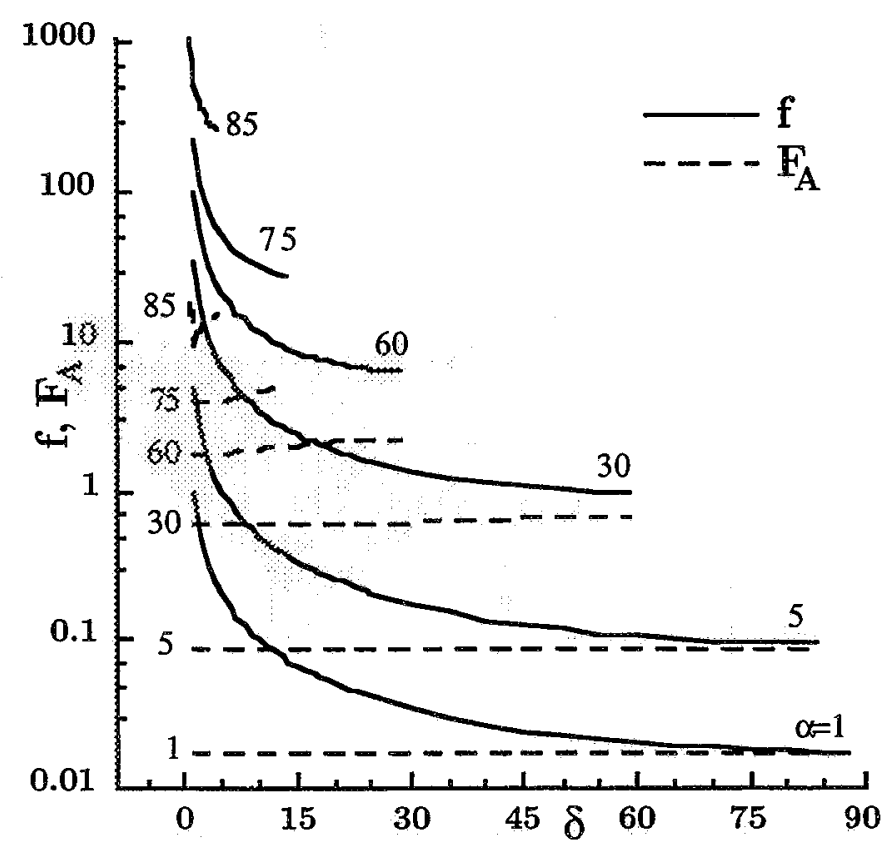

Figure 2.3: The parameters $f$ and $F_{A}$ as a function of $\delta$ for various $\alpha$ (deg).

be determined geometrically

$$
F_{A}=\frac{f^{2} \sin ^{2} \delta}{\tan \alpha}\left(1-\left(\frac{2 \delta-\sin 2 \delta}{1-\cos 2 \delta}\right) \tan \alpha\right)
$$

Figure 2.3 shows $F_{A}$ and $f$ plotted against $\delta$ for a variety of corner half-angles $\alpha$. Note that $F_{A}$ is an $O(1)$ quantity for intermediate values of $\alpha(\pi / 12 \lesssim \alpha \lesssim 5 \pi / 12)$ and shows weak functional dependence on $\delta$ for fixed $\alpha$. It can be shown further that $1 \leq F_{A} / \tan \alpha \lesssim 4 / 3$ for all $\alpha$ and $\delta$, and thus, the length scale $y^{\prime} \sim H^{\prime} \tan \alpha$ is appropriate for all $\alpha$ and $\delta$ and that, in general, $F_{A} \sim \tan \alpha$. Note also that $f$ behaves like $1 / \delta$ for $\delta \rightarrow 0$.

Regardless of the technique employed to solve eq 2.29 the resulting form for $\left\langle w_{o}\right\rangle$ will be

$$
\left\langle w_{o}\right\rangle=-F_{i} h^{2} \frac{\partial P_{o}}{\partial z}=-F_{i} \frac{\partial h}{\partial z}
$$

where $F_{i}$ is some positive geometric function of $\alpha$ and $\delta$. It is interesting that viscous resistance $\left(\sim h^{-2}\right)$ is balanced by capillary forces $\left(\sim h^{-2} h_{z}\right)$ in such a way as to leave $\left\langle w_{o}\right\rangle$ only dependent on the slope of the interface $h_{z}$, and not on $h$. As a result, non-zero velocities are possible for $h=0$ provided $h_{z} \neq 0$. 
Noting that $A \propto h^{2}$, eq 2.36 may be substituted into eq 2.23 which, after some rearrangement, yields the leading order governing equation for the corner flow problem in the $\epsilon^{2} \ll 1$ and $f \epsilon^{2} \ll 1$ limits

$$
2 \frac{\partial h}{\partial t}=F_{i}\left(2\left(\frac{\partial h}{\partial z}\right)^{2}+h \frac{\partial^{2} h}{\partial z^{2}}\right)
$$

In this way, the meniscus centerline height $h$ becomes the principal dependent variable of the problem. Eq 2.37 also appears in the literature where it describes unsteady, nonlinear heat conduction of the form $T_{t}=\left(T^{1 / 2} T_{z}\right)_{z}[17][25]$.

In addition to the selection of boundary conditions, solutions to eq 2.37 need only $F_{i}$ to begin. Generally speaking, Ransohoff and Radke [11] and Ayyaswamy et al. [9] have determined $F_{i}$ for this problem. ${ }^{4}$ They first use a finite element scheme to solve eq 2.29 for $w_{o}$. They then integrate over the cross sectional area to determine $\left\langle w_{0}\right\rangle$ and the tabulated results are presented in terms of either a dimensionless flow resistance, $\beta$, in the case of $[11]$, or a dimensionless friction factor coefficient, $K$, as in the case of [9]. Ransohof and Radke [12] also employ eq 2.37, as do Dong and Chatzis [17]. Both employ $\beta$ in its solution. In [12] the continuity equation in the general form of eq 2.37 is solved numerically for a problem relating to snap-off in constricted pores while in [17] a similarity solution is found applicable to imbibition in square capillary tubes. The usefulness of these solutions is that they are only restricted to $\epsilon^{2} \ll 1$ (slender fluid column), $\epsilon^{2} f \ll 1$ (slight curvature along $z$-axis), and $\mathcal{R} \lesssim O\left(\epsilon^{2}\right)$ (small inertia). For many practical systems $f \sim O(1)$ and the second constraint is naturally satisfied by the first.

An analytic form for $F_{i}$, however, is still desirable and under certain restricted cases further asymptotic analyses are possible to determine its functional form and hence the leading order behavior of the corner flow, namely $h(z, t)$. It will be demonstrated that the scaling presented here renders $F_{i}$ a weak function of $\alpha$ and $\delta$ such that $1 / 8 \lesssim F_{i} \leq 1 / 6$ for all values of $\alpha$ and $\delta$. The utility of this fact should become apparent in subsequent sections where problems of practical interest are addressed.

\footnotetext{
${ }^{4}$ Lenormand and Zarcone [26] and Trefethen [27] use a hydraulic diameter approach in effect to determine $F_{i}$, the utility of which is discussed in [11]. Lenormand and Zarcone ([26], Appendix 2) also anticipate the work of [17].
} 


\subsection{Solutions for $F_{i}$}

In this section asymptotic techniques are applied to eq 2.29 for the limiting cases of small $(I)$ and large $(I I)$ corner angle. ${ }^{5}$ The latter is solved for the free $(I I)$ and captive $\left(I I_{c}\right)$ surface conditions. A form for $F_{i}$ is also determined using the hydraulic diameter approach $\left(I I I, F_{h y d}\right)$. In addition, two exact analytic expressions for $F_{i}$ are possible for the special cases of $\theta=\alpha=\pi / 4$ and for $\theta=\pi / 3, \alpha=\pi / 6$. These results are then compared to the numerical results of Ransohoff and Radke [11] who determine $\beta$ for a wide range of $\alpha, \theta$, and surface conditions satisfying the ConcusFinn condition. From the definition of $\beta$, it can be shown that

$$
F_{i}=\frac{f^{2}}{\beta \sin ^{2} \alpha}
$$

For the free surface problem, the numerical results of Ayyaswamy et al. [9], who calculate a friction factor coefficient $K$ for the problem of gravity driven flow in triangular grooves, are also applicable and are used to compare with $F_{i}$. It can be shown that

$$
F_{i}=\frac{8}{K}\left(\frac{F_{A}}{f \sin \delta}\right)^{2}
$$

An outline of the general asymptotic solution is provided for case $I$ only. The analytical details of the solutions for $F_{I I}, F_{I I_{c}}$, and $F_{h y d}$ are relegated to Appendix A.2. Though only the zeroeth order terms are applicable due to the nature of eq 2.29 , higher order terms are retained for completeness.

Case I. $F_{I}$, Small corner angle solution, $\tan ^{2} \alpha \ll 1$

In the limit $\alpha^{2} \ll 1$, an expansion for $w_{\circ}$ may be written

$$
w_{o}=w_{o_{0}}+w_{o_{1}} \tan ^{2} \alpha+w_{o_{2}} \tan ^{4} \alpha+\ldots
$$

and when substituted into eq 2.29 may be solved for $w_{o}$ to any desired order subject to the no-slip and symmetry conditions. This procedure yields

$$
w_{o}=\frac{1}{2 \cos 2 \alpha} \frac{\partial P_{o}}{\partial z}\left(y^{2}-x^{2}\right)
$$

The average velocity, $\left\langle w_{o}\right\rangle_{I}$, may in turn be determined using

$$
\left\langle w_{o}\right\rangle_{I}=-\frac{\tan \alpha}{h^{2} F_{A} \cos 2 \alpha} \frac{\partial P_{o}}{\partial z} \int_{0}^{y_{m}} \int_{y}^{S_{o}}\left(y^{2}-x^{2}\right) d x d y
$$

\footnotetext{
${ }^{5}$ These cases were anticipated in [11] yet no analytical treatment of them has appeared in the literature to my knowledge.
} 
Upon substitution of the quantities $P_{o}, S_{o}, y_{m}$ and $f$ from eqs 2.31-2.34 the above integration yields

$$
\left\langle w_{o}\right\rangle_{I}=-F_{I} \frac{\partial h}{\partial z}
$$

where $F_{I}$ replaces $F_{i}$ of eq 2.37 for the limiting case of small corner angle, $\tan ^{2} \alpha \ll 1$, and is given by

$$
\begin{gathered}
F_{I}=\frac{2 f}{F_{A} \cos 2 \alpha}\left\{\frac{\sin \delta}{6}-\frac{f}{4}\left[\delta-2 \sin \delta+\frac{\sin 2 \delta}{2}\right]-\frac{f^{2}}{2}\left[\delta-2 \sin \delta+\frac{\sin 2 \delta}{2}+\frac{\sin ^{3} \delta}{3 \sin ^{2} \alpha}\right]\right. \\
-f^{3}\left[\frac{5}{16}\left(\delta+\frac{\sin 2 \delta}{2}\right)\left(1-\frac{\cot ^{2} \alpha}{5}\right)-\frac{2}{3} \sin \delta+\frac{\sin \delta \cos ^{3} \delta}{24}\left(1+3 \cot ^{2} \alpha\right)+\right. \\
\left.\left.\frac{\sin ^{3} \delta}{6 \sin ^{2} \alpha}-\frac{\sin ^{4} \delta \cot ^{3} \alpha}{12}\right]\right\}
\end{gathered}
$$

Some limiting values of $F_{I}$ are

$$
\begin{gathered}
F_{I}\left(\delta^{2} \ll 1\right)=\frac{1}{6}+\frac{\alpha^{2}}{3}+\frac{\alpha \delta}{90}+O\left(\alpha^{4}\right) \\
F_{I}(\delta=\pi / 2-\alpha)=\frac{1}{6}+\frac{\alpha}{3}\left(1-\frac{5 \pi}{16}\right)+\frac{\alpha^{2}}{2}\left(3-\frac{5 \pi}{12}-\frac{5 \pi^{2}}{48}\right)+O\left(\alpha^{3}\right)
\end{gathered}
$$

which reveal that in the limit $\tan ^{2} \alpha \rightarrow 0, F_{I} \rightarrow 1 / 6$, and thus $\left\langle w_{o}\right\rangle=-h_{z} / 6$. Note also that $\delta=\pi / 2-\alpha$ when $\theta=0$.

Case II. $F_{I I}$, Large corner angle solution, $\Omega^{2} \ll 1, \delta^{2} \ll 1$

An asymptotic solution for $F_{i}$ is also possible when $\alpha$ approaches $\pi / 2$. Introducing $\Omega \equiv \pi / 2-\alpha$, the case of the large corner angle limit is given by $\Omega^{2} \ll 1$. For simplicity of presentation, $\phi \equiv \delta / \Omega$. The limit $\Omega^{2} \ll 1$ automatically implies $\delta^{2} \ll 1$ when the Concus-Finn condition is satisfied.

A. Free Surface Condition. A similar analysis as the above yields the full expression for $F_{i}=F_{I I}$ to $O\left(\Omega^{2}\right)$

$$
\begin{gathered}
F_{I I}=\frac{70-126 \phi+77 \phi^{2}-16 \phi^{3}}{35(\phi-2)^{2}(3-2 \phi)}+\frac{\Omega^{2}}{(\phi-2)^{2}(3-2 \phi)^{2}} \\
\times\left(12-\frac{458 \phi}{15}+\frac{449 \phi^{2}}{15}-\frac{284 \phi^{3}}{21}+\frac{1613 \phi^{4}}{700}+\frac{57 \phi^{5}}{350}-\frac{16 \phi^{6}}{225}\right)+O\left(\Omega^{4}\right)
\end{gathered}
$$


with the limiting behavior

$$
\begin{gathered}
F_{I I}(\phi=0)=\frac{1}{6}+\frac{\Omega^{2}}{3}+O\left(\Omega^{4}\right) \\
F_{I I}(\phi=1)=\frac{1}{7}+\frac{49}{180} \Omega^{2}+O\left(\Omega^{4}\right) \\
F_{I I}(\phi=-1)=\frac{289}{1575}+\frac{554783}{1417500} \Omega^{2}+O\left(\Omega^{4}\right)
\end{gathered}
$$

B. Captive Surface Condition. This solution follows similarly for the case $\Omega^{2} \ll 1$, but with a captive (no-slip) surface condition. The boundary condition on $x=S_{0}$ becomes $w=0$. The full expression for $F_{i}=F_{I I_{c}}$ to $O\left(\Omega^{2}\right)$ is

$$
\begin{gathered}
F_{I I_{c}}=\frac{70-126 \phi+77 \phi^{2}-16 \phi^{3}}{140(\phi-2)^{2}(3-2 \phi)}+ \\
\frac{\Omega^{2}}{(\phi-2)^{2}(3-2 \phi)^{2}}\left[\frac{3}{2}-\frac{10 \phi}{3}+\frac{73 \phi^{2}}{30}-\frac{34 \phi^{3}}{105}-\frac{67 \phi^{4}}{175}+\frac{29 \phi^{5}}{175}-\frac{4 \phi^{6}}{225}\right]+O\left(\Omega^{4}\right)
\end{gathered}
$$

with limiting behavior

$$
\begin{gathered}
F_{I I_{c}}(\phi=0)=\frac{1}{24}+\frac{1}{24} \Omega^{2}+O\left(\Omega^{4}\right) \\
F_{I I_{c}}(\phi=1)=\frac{1}{28}+\frac{13}{315} \Omega^{2}+O\left(\Omega^{4}\right) \\
F_{I I_{c}}(\phi=-1)=\frac{289}{6300}+\frac{11063}{354375} \Omega^{2}+O\left(\Omega^{4}\right)
\end{gathered}
$$

\section{Case III. $F_{\text {hyd }}$, Hydraulic diameter solution}

A. Free surface condition. Application of the hydraulic diameter concept for this problem gives the result

$$
F_{h y d}=\frac{1}{8}\left[\frac{1-\left(\frac{2 \delta-\sin 2 \delta}{1-\cos 2 \delta}\right) \tan \alpha}{1-\left(\frac{1-\cos \delta}{\sin \delta}\right) \tan \alpha}\right]^{2}
$$

with limiting cases

$$
\begin{gathered}
F_{h y d}(\delta=0)=\frac{1}{8} \\
F_{h y d}\left(\Omega^{2} \ll 1, \phi=1\right)=\frac{1}{288}+\frac{13 \Omega^{2}}{2880}+O\left(\Omega^{4}\right)
\end{gathered}
$$




$$
F_{\text {hyd }}\left(\Omega^{2} \ll 1, \phi=-1\right)=\frac{25}{32}+\frac{49 \Omega^{2}}{192}+O\left(\Omega^{4}\right)
$$

B. Captive surface condition. For the case of the captive surface condition the hydraulic approach yields the full expression

$$
F_{h y d_{c}}=\frac{1}{8}\left[\frac{1-\left(\frac{2 \delta-\sin 2 \delta}{1-\cos 2 \delta}\right) \tan \alpha}{1-\left(\frac{1-\cos \delta}{\sin \delta}\right) \tan \alpha}\right]^{2}\left[\frac{1}{1+\frac{\delta \sin \alpha}{\sin \delta}}\right]^{2}
$$

with the limiting behavior

$$
\begin{gathered}
F_{h y d_{c}}(\delta=0)=\frac{1}{8(1+\sin \alpha)^{2}} \\
F_{h y d_{c}}\left(\Omega^{2} \ll 1, \phi=1\right)=\frac{1}{1152}+\frac{49 \Omega^{2}}{34560}+O\left(\Omega^{4}\right) \\
F_{h y d_{c}}\left(\Omega^{2} \ll 1, \phi=-1\right)=\frac{25}{128}+\frac{\Omega^{2}}{768}+O\left(\Omega^{4}\right)
\end{gathered}
$$

Case IV. $F_{I V}$, Exact solution for a free surface, $\alpha=\theta=\pi / 4$

The analytic solutions for $F_{i}$ are adapted from classic solutions of viscous flows in noncircular ducts [28]. For the case $\alpha=\pi / 4, \theta=\pi / 4, \delta=0$, and the free surface (zero shear stress) boundary condition it can be shown that

$$
F_{I V}=-\frac{1}{3}\left[1-\frac{192}{\pi^{5}} \sum_{i=1,3,5 \ldots}^{\infty} \frac{\tanh i \pi / 2}{i^{5}}\right]=0.14057 \ldots
$$

The actual flow domain here is the half section of a square duct bisected along the diagonal.

Case V. $F_{V}$, Exact solution for a captive surface, $\alpha=\pi / 6, \theta=\pi / 3$

This is the solution for viscous flow through a duct with equilateral triangular cross section. The boundary condition on the fluid interface is the captive (no slip) surface condition [28]. Again, for this case, $\delta=0$ and

$$
F_{V}=-\frac{1}{15}
$$

Summary of solutions for $F_{i}$ 
Table 2.2 summarizes the above results for $F_{i}$ and includes the numerical values of [11]. Decimal values for $F_{i}$ listed in the table are values determined using the numerical value of $\beta$ and eq 2.38. Fractions or '...' values are either asymptotic or exact analytical results.

Observation of the tabulated quantities reveals that, though $\beta$ yields values from $O(1)$ to $\infty$ for the full range of $\theta$ and $\alpha$ satisfying the Concus-Finn condition, $F_{i}$ is banded and $O(1)$ particularly for the more important free surface condition where $1 / 8 \lesssim F_{i} \leq 1 / 6$. This is confirmation that the governing equations are scaled appropriately by the quantities of Table 2.1 and that $\sin ^{2} \alpha$ and $f$ appearing in $W$ capture the respective geometric components of viscous resistance and capillary driving force for the flow. For the case of the Concus-Finn condition with a free surface $\beta \geq 6$. If $\theta=\pi / 2-\alpha$, then $\beta=\infty$.

In all cases $F_{h y d}<F_{i}$ and in the extreme cases, $F_{i}$ can be as much as 40 times larger than $F_{\text {hyd }}(\alpha=\pi / 2$ and $\theta=0)$, or differ by as little as $10 \%(\alpha=\theta=\pi / 4)$. The hydraulic diameter approach does not nearly capture the nature of the correct $F_{i}$ over the full range of $\alpha$ and $\theta$, and though the prospect of an analytic form for $F_{i}$ for all $\alpha$ and $\theta$ is attractive, the results/warnings given by Ransohof and Radke [11] should be heeded and the hydraulic diameter approach should not be followed.

Table 2.3 compares $F_{i}$ to $K$ determined numerically by Ayyaswamy et al. [9] for a selection of $\alpha$ and $\theta$. It can be shown from the asymptotic analysis that for $\alpha^{2} \ll 1$, $K=48+O\left(\alpha^{2}\right)$. In the large angle limit $\Omega^{2} \ll 1, K=\left(8 / F_{i}\right)(1-\delta / 3 \Omega+O(\Omega \delta))$. Note also that if $\delta=0, F_{i}=8 / K$. In contrast to $\beta, K(\alpha, \delta)$ is a banded function similar to $F_{i}$, and, as seen from Table 2.3 , takes values in the range $30 \lesssim K \lesssim 57$ for the values of $\alpha$ and $\delta$ listed. ${ }^{6}$ The primary differences between $K$ and $F_{i}$ are sourced in the length scale choice for the cross-How problem. Ayyaswamy et al. [9] use the distance of the contact line from the corner to scale all lengths whereas $h$ (or $H$ ) is used in this analysis. The fact that $F_{i}$ is more tightly banded than is $K$ for the full range of $\alpha$ and $\delta$, and that $F_{i} \sim O(1)$ while $K \sim O(10)$, indicates that $H$ is a more correct choice for the cross-sectional length scale of the problem.

Figure 2.4 displays the results of Tables 2.2 and 2.3 for systems with a free surface. It is readily observed that $F_{i}$ falls within a narrow band of values for all $\alpha$ and $\delta$. In Figure 2.5, $F_{i}$ is plotted against $\alpha$ for the captive surface condition. The banded behavior of $F_{i}$ for this problem is reduced. The asymptotic solutions for $\Omega^{2} \ll 1$ are

\footnotetext{
${ }^{6} K$ may yield values less than 30 for $\alpha>60^{\circ}$. For example, using the value of $F_{i}$ determined from the value of $\beta$ for $\alpha=72^{\circ}, \theta=0$, eq 2.39 may be employed to show $K=27.9$ for this case. However, $F_{i}$ values determined from the numerical values of $\beta$ and $K$ show a discrepancy of approximately $3 \%$ between the two numerical results at low contact angle (compare $F_{i}$ in Tables 2.2 and 2.3 for $\alpha=30^{\circ}$ ).
} 


\begin{tabular}{ll|lll|lll|}
\cline { 3 - 8 }$\alpha$ & & \multicolumn{2}{|c|}{ Free Surface $\left(\eta_{R R}=0\right)$} & \multicolumn{3}{c|}{ Captive Surface $\left(\eta_{R R}=10^{8}\right)$} \\
\cline { 3 - 8 } & $\beta$ & $F_{i}$ & $F_{h y d}$ & $\beta$ & $F_{i}$ & $F_{h y d}$ \\
\hline 0 & 0 & $6^{*}$ & $1 / 6$ & $1 / 8$ & $6^{*}$ & $1 / 6$ & $1 / 8$ \\
10 & 0 & 9.981 & 0.146 & 0.0548 & 13.6 & 0.108 & 0.0353 \\
15 & 0 & 12.95 & 0.141 & 0.0382 & 19.91 & 0.0914 & 0.0209 \\
30 & 0 & 31.07 & 0.129 & 0.0154 & 65.02 & 0.0615 & 0.00600 \\
36 & 0 & 46.67 & 0.127 & 0.0115 & 108.1 & 0.0544 & 0.00405 \\
45 & 0 & 93.93 & 0.124 & 0.00790 & 248.8 & 0.0469 & 0.00248 \\
60 & 0 & 443.0 & 0.126 & 0.00498 & 1411 & 0.0395 & 0.00137 \\
72 & 0 & 3185 & 0.131 & 0.00395 & 11390 & 0.0367 & 0.00102 \\
90 & 0 & $\infty^{*}$ & $1 / 7$ & $1 / 8$ & $\infty^{*}$ & $1 / 28$ & $1 / 32$ \\
\hline 30 & 0 & 31.07 & 0.129 & 0.154 & 65.02 & 0.0615 & 0.00600 \\
30 & 5 & 30.89 & 0.132 & 0.0205 & 65.64 & 0.0619 & 0.00813 \\
30 & 10 & 31.94 & 0.133 & 0.0261 & 68.24 & 0.0624 & 0.0106 \\
30 & 20 & 38.10 & 0.136 & 0.0396 & 81.58 & 0.0634 & 0.0166 \\
30 & 30 & 54.09 & 0.138 & 0.0558 & 115.9 & 0.0644 & 0.0241 \\
30 & 36 & 75.20 & 0.139 & 0.0671 & 161.3 & 0.0649 & 0.0292 \\
30 & 45 & 165.2 & 0.141 & 0.0861 & 355.5 & 0.0656 & 0.0380 \\
30 & 60 & $\infty^{*}$ & - & $1 / 8$ & $\infty^{*}$ & $1 / 15$ & $1 / 18$ \\
\hline 45 & 0 & 93.9 & 0.124 & 0.00790 & 248.8 & 0.0469 & 0.00248 \\
45 & 5 & 93.99 & 0.127 & 0.0127 & 253.2 & 0.0473 & 0.00408 \\
45 & 10 & 100.2 & 0.129 & 0.0191 & 270.8 & 0.0478 & 0.00622 \\
45 & 20 & 139.0 & 0.133 & 0.0373 & 374.9 & 0.0493 & 0.0124 \\
45 & 30 & 290.7 & 0.136 & 0.0640 & 782. & 0.0506 & 0.0217 \\
45 & 36 & 698.2 & 0.138 & 0.0850 & 1881 & 0.0512 & 0.0291 \\
45 & 45 & $\infty^{*}$ & $0.14057 \ldots$ & $1 / 8$ & $\infty^{*}$ & - & $1 /(12+8 \sqrt{2})$ \\
\hline
\end{tabular}

Table 2.2: Values of $\beta, F_{i}$, and $F_{\text {hyd }}$. An asterisk * indicates that the value was determined by eq 2.38 using $F_{i}$. The parameter $\eta_{R R}$ is the surface viscosity coefficient [11]. The free surface condition uses $\eta_{R R}=0$, and for these numerical solutions, the captive surface, $\eta_{R R} \equiv \infty$, uses the approximation $\eta_{R R}=10^{8}$. 


\begin{tabular}{|c|c|c|c|c|c|c|}
\hline & $K$ & $\overline{F_{i}}$ & $K$ & $\overline{F_{i}}$ & $\bar{K}$ & $\overline{F_{i}}$ \\
\hline$\theta$ & \multicolumn{2}{|c|}{$\alpha=5^{\circ}$} & \multicolumn{2}{|c|}{$\alpha=10$} & \multicolumn{2}{|c|}{$\alpha=20$} \\
\hline 0.1 & 45.691 & 0.159 & 43.784 & 0.152 & 40.588 & 0.140 \\
\hline 5 & 46.295 & 0.159 & 44.951 & 0.152 & 42.814 & 0.140 \\
\hline 10 & 46.798 & 0.159 & 45.927 & 0.152 & 44.686 & 0.141 \\
\hline 20 & 47.539 & 0.159 & 47.376 & 0.153 & 47.483 & 0.142 \\
\hline 30 & 48.031 & 0.160 & 48.354 & 0.154 & 49.407 & 0.144 \\
\hline 40 & 48.356 & 0.160 & 49.017 & 0.155 & 50.758 & 0.145 \\
\hline 50 & 48.559 & 0.161 & 49.454 & 0.156 & 51.712 & 0.147 \\
\hline 60 & 48.665 & 0.162 & 49.714 & 0.157 & 52.367 & 0.149 \\
\hline 70 & 48.686 & 0.163 & 49.824 & 0,158 & 52.778 & 0.151 \\
\hline 80 & 48.625 & 0.164 & 49.793 & 0.160 & - & - \\
\hline \multirow[t]{2}{*}{85} & 48.563 & 0.164 & - & - & - & - \\
\hline & \multicolumn{2}{|c|}{$\alpha=30$} & \multicolumn{2}{|c|}{$\alpha=40$} & \multicolumn{2}{|c|}{$\alpha=50$} \\
\hline 0.1 & 37.797 & 0.132 & 35.215 & 0.128 & 32.797 & 0.126 \\
\hline 5 & 41.053 & 0.133 & 39.544 & 0.129 & 38.346 & 0.128 \\
\hline 10 & 43.790 & 0.134 & 43.149 & 0.130 & 42.863 & 0.130 \\
\hline 20 & 47.864 & 0.136 & 48.432 & 0.133 & 49.262 & 0.134 \\
\hline 30 & 50.681 & 0.138 & 52.058 & 0.136 & 53.563 & 0.138 \\
\hline 40 & 52.706 & 0.140 & 54.703 & 0.139 & 56.720 & 0.141 \\
\hline 50 & 54.201 & 0.142 & 56.762 & 0.141 & - & - \\
\hline \multirow[t]{2}{*}{60} & 55.316 & 0.144 & - & - & - & - \\
\hline & \multicolumn{2}{|c|}{$\alpha=60$} & & & \multicolumn{2}{|c|}{$\alpha=90(\delta=0)$} \\
\hline \multirow{5}{*}{$\begin{array}{c}0.1 \\
5 \\
10 \\
20\end{array}$} & 30.449 & 0.127 & & & $48^{*}$ & $1 / 6$ \\
\hline & 37.615 & 0.130 & & & \multicolumn{2}{|c|}{$\alpha=90(\theta=0)$} \\
\hline & 43.214 & 0.133 & & & $37.3^{*}$ & $1 / 7$ \\
\hline & 50.571 & 0.140 & & & $\alpha=0$ & ( all $\theta$ ) \\
\hline & 55.315 & 0.144 & & & $48^{*}$ & $1 / 6$ \\
\hline
\end{tabular}

Table 2.3: Values of $K$ and $F_{i}$ for the free surface problem. An asterisk * indicates that the value of $K$ was determined by eq 2.39 using the asymptotically determined value of $F_{i}$. 


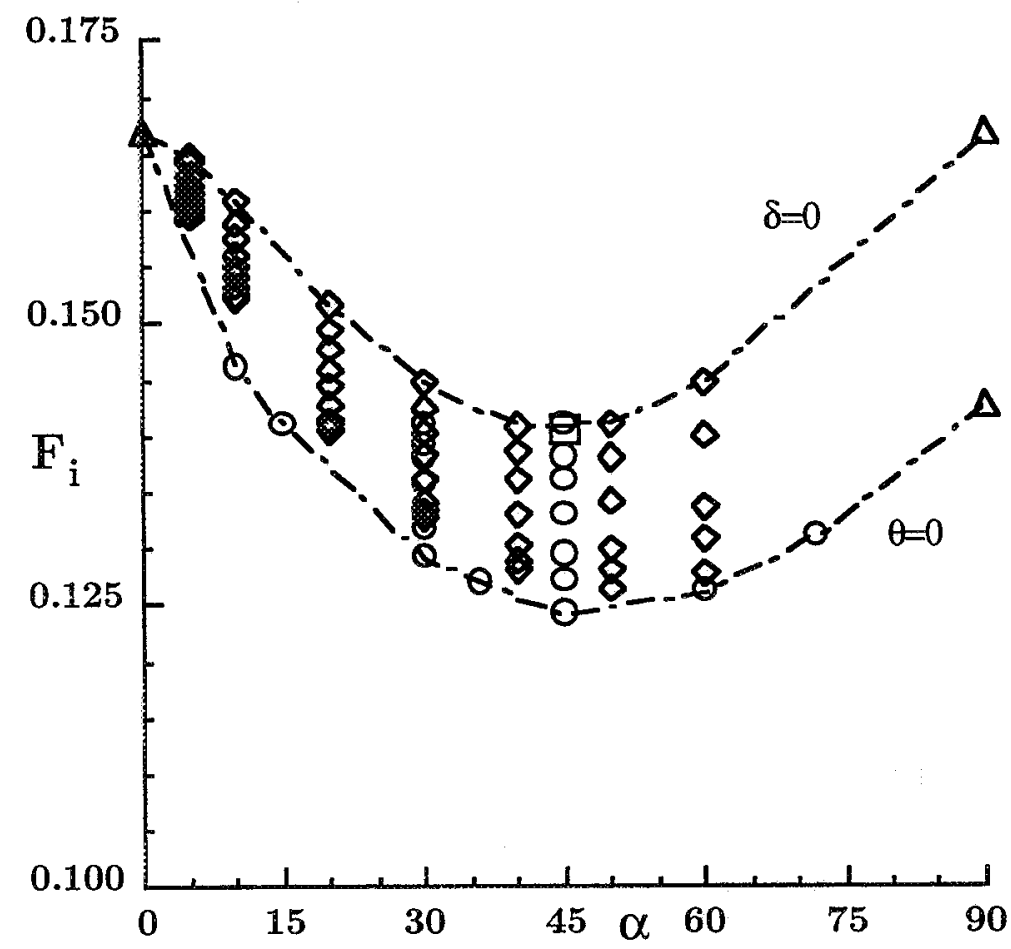

Figure 2.4: $F_{i}(\alpha, \theta)$. For the free surface condition the value of $F_{i}$ falls between the curves for $\delta=0$ and $\theta=0$ approximated by dashed lines. For all $\alpha$ and $\delta$, $1 / 8 \lesssim F_{i} \leq 1 / 6$. Data from Tables 2.2 and 2.3. $\bigcirc \beta, \diamond K, \triangle$ asymptotic, $\square$ Case IV.

extended across the full domain of $\alpha$. From this point on only systems satisfying the free surface condition as well as the Concus-Finn condition will be discussed.

With $F_{i}$ determined analytically for cases I or II above, or by noting that $1 / 8 \lesssim F_{i} \leq$ $1 / 6$ is a weak function of $\alpha$ and $\theta$-the precise value of which may be determined numerically [9][11]-eq 2.37 can now be treated using asymptotic and/or other analytical techniques. Introducing $\tau=F_{i} t / 2$ transforms eq 2.37 to

$$
\frac{\partial h}{\partial \tau}=2\left(\frac{\partial h}{\partial z}\right)^{2}+h \frac{\partial^{2} h}{\partial z^{2}}
$$




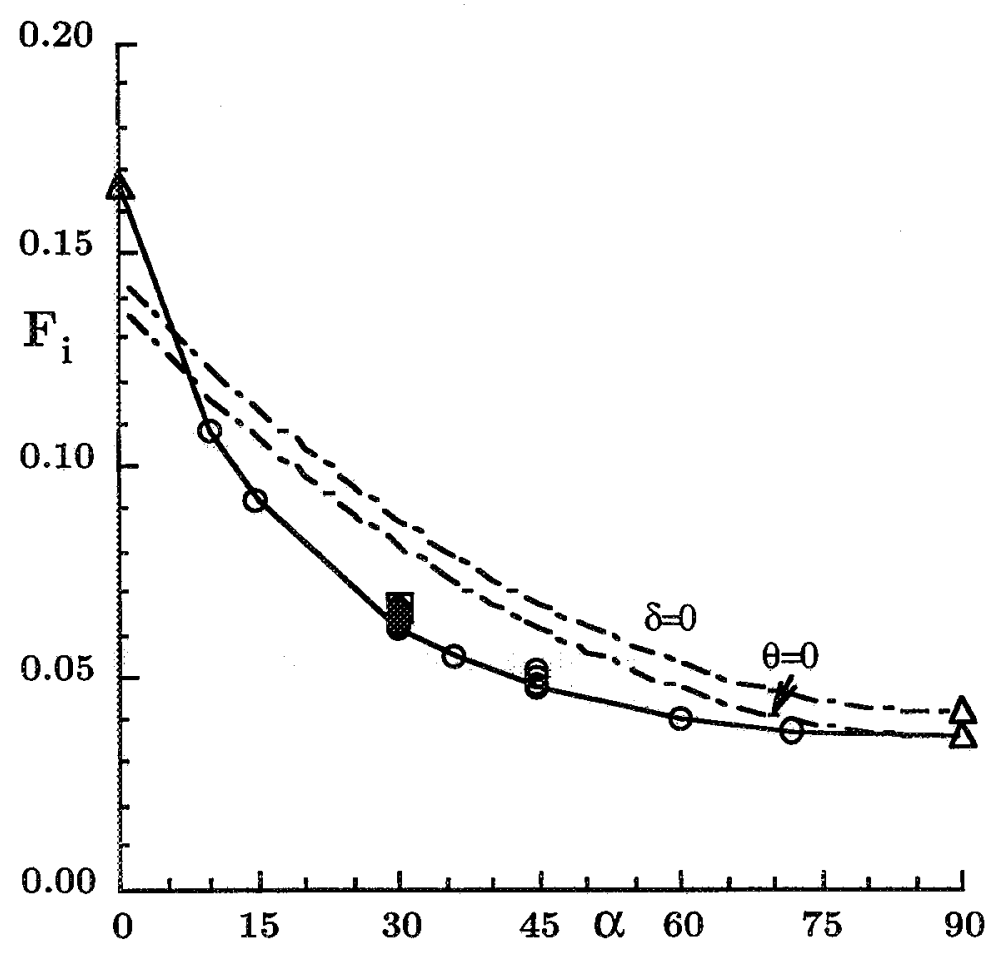

Figure 2.5: $F_{i}(\alpha, \theta)$ for the captive surface condition. Dashed lines are asymptotic results for $\Omega^{2} \ll 1$ for $\delta=0$ and $\theta=0$. Data from Table 2.2. $\bigcirc \beta$ (solid line $\theta=0$ ), $\triangle$ asymptotic, $\square$ Case V. 


\subsection{Steady Solution}

The steady solution to eq 2.51 is

$$
h=\left(B_{1}+B_{2} z\right)^{1 / 3}
$$

where $B_{1}$ and $B_{2}$ are given by the boundary conditions. By specifying $h\left(z_{1}, \tau\right)$ and $h\left(z_{2}, \tau\right), B_{1}$ and $B_{2}$ are determined, and eq 2.36 may be solved and substituted into $\dot{Q}=A\langle w\rangle$ which gives the maximum flow rate due to capillary pumping for a fiuid column of length $\mathcal{L}$.

\subsection{Infinite Column Solution}

The principle dependent variable $h$ may be expanded as

$$
h=h_{o}+\epsilon h_{1}+\epsilon^{2} h_{2}+\ldots
$$

Eq 2.51 includes terms through $O(\epsilon)$. On substitution of eq 2.53 into eq 2.51, standard asymptotics yields the $O(1)$ equation

$$
\frac{\partial h_{o}}{\partial \tau}=2\left(\frac{\partial h_{o}}{\partial z}\right)^{2}+h_{o} \frac{\partial^{2} h_{o}}{\partial z^{2}}
$$

and the $O(\varepsilon)$ equation

$$
\frac{\partial h_{1}}{\partial \tau}=4 \frac{\partial h_{o}}{\partial z} \frac{\partial h_{1}}{\partial z}+h_{o} \frac{\partial^{2} h_{1}}{\partial z^{2}}+h_{1} \frac{\partial^{2} h_{o}}{\partial z^{2}}
$$

One (of many) simple solution may be obtained for the corner flow by noting that $h_{o}=$ const is a solution to eq 2.54. This solution describes an interface at steady state in a corner of infinite extent. Then, by solving eq $2.55, h$ may be determined to $O(\epsilon)$ as

$$
h=h_{o}+\epsilon \exp \left[-\xi^{2} h_{o} \tau\right] C_{1} \cos \left(\xi z+C_{2}\right)+O\left(\epsilon^{2}\right)
$$

where $\xi$ is the dimensionless wave number $\left(\xi=\xi^{\prime} / L\right)$ of some disturbance and $C_{1}$ and $C_{2}$ are constants given by the boundary conditions. From this result several useful characteristics for the flow may be distinguished.

A key feature of eq 2.56 is the emergence of a time constant. When redimensionalized, the decaying exponent reveals

$$
\xi^{2} h_{o} \tau \sim \epsilon^{2} \frac{F_{i} \sin ^{2} \alpha}{f} \frac{\sigma t}{\mu H}=\epsilon^{2}\left(\mathcal{F} C a_{t}\right)^{-1}
$$




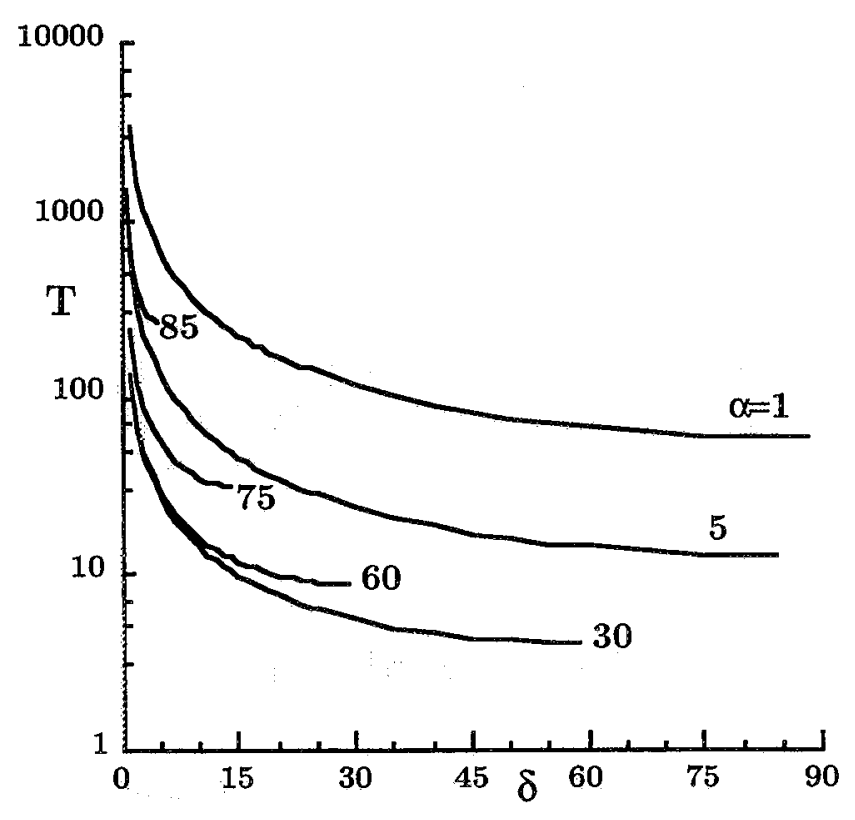

Figure 2.6: Geometric time constant $T(\alpha, \delta)$.

where $H$ is the unperturbed height of the meniscus. $\mathcal{F}=f / F_{i} \sin ^{2} \alpha$ and $C a_{t}=$ $H \mu / \sigma t$, the latter being a time-dependent Capillary number.

Recalling that $F_{i} \approx 1 / 7$, for given base state fluid height $H$ and fluid properties $\sigma / \mu$, the decay function will be $\propto t \sin ^{2} \alpha / f$. Thus, the time constant will be $\alpha$ $f / \sin ^{2} \alpha$. The relationship $T=f / \sin ^{2} \alpha$ is plotted in Figure 2.6 for a variety of $\alpha$ and $\delta$, for cases satisfying the Concus-Finn condition. As can be seen from the figure, the time constant rapidly increases as $\delta \rightarrow 0$, particularly for $\delta \lesssim 10^{\circ}$ where $T \sim 1 /(\delta \sin 2 \alpha)$. The lowest value for $T$ is achieved for $\alpha=30^{\circ}, \delta=\pi / 2-\alpha$. This remains true when the slight variation of $F_{i}$ with $\alpha$ and $\delta$ is considered. The function $T$ is the ratio of geometric quantities which characterize the viscous $\left(\sim 1 / \sin ^{2} \alpha\right)$ and capillary forces $(\sim 1 / f)$. Changes in fluid behavior due to contact angle are effected solely through the latter.

The contact line velocity can be determined from eq 2.56. The associated Capillary number may also be estimated which shows an initial maximum value of $C a_{c l} \sim \epsilon^{3} / \mathcal{F}$ that decreases exponentially with time. This value of $C a$ is indeed small and supports the use of the static contact angle slip condition at the contact line, eq 2.19 .

It can be shown that the inclusion of gravity along the $x$-direction $\left(g_{x}\right)$ modifies the result of eq 2.56 to produce

$$
h=h_{o}+\epsilon \exp \left[-\xi^{2} h_{o}\left(1+h_{o}^{2} B o_{H}\right) \tau\right] C_{1} \cos \left(\xi z+C_{2}\right)
$$


The form of eq 2.56 is recovered for $B o_{H} \equiv f \rho g_{x} H^{2} / \sigma=0$ (see Appendix A.4) and the column is unstable for $B o_{H}<-1\left(h_{o}=1\right)$. Order of magnitude predictions of settling times for slightly disturbed systems are possible by suitable selection of $H$ and $L$.

\subsection{Similarity Solutions}

Using similarity transformations eq 2.51 may be solved for several problems of practical interest. In this section a generalized similarity ODE for $h(z, t)$ is derived. Then solutions are found for the special cases of capillary rise, a spreading drop, and constant flow. All of these cases possess an advancing meniscus where $h=0$ at the tip. The location of the tip on the $z$-axis is denoted by $\mathcal{L}=\mathcal{L}(\tau)$. These three problems were alluded to in [17] and [26] and the problem of capillary rise was solved in the former. The special case of capillary rise is rederived along with the other new solutions.

The introduction of

$$
h=C_{1} \tau^{a} F(\eta) \quad \eta=C_{2} z \tau^{b} \quad \mathcal{L}=\eta_{t i p} C_{2}^{-1} \tau^{-b}
$$

where $C_{1}, C_{2}$, and $\eta_{t i p}$ are constants, transforms the governing eq 2.51 to

$$
F F_{\eta \eta}+2 F_{\eta}^{2}-\frac{\tau^{-1-a-2 b}}{C_{1} C_{2}^{2}}\left[a F+b \eta F_{\eta}\right]=0
$$

The case of $a=b=0$ is the steady state problem which yields a simple analytical solution, eq 2.52. Similarity in eq 2.60 is achieved when

$$
b=-\frac{(1+a)}{2}
$$

giving

$$
F F_{\eta \eta}+2 F_{\eta}^{2}-\frac{1}{C_{1} C_{2}^{2}}\left[a F+b \eta F_{\eta}\right]=0
$$

The difficulty now faced is one of identifying practical boundary conditions which yield to the similarity form and supply values for $a$ and $b$. The constants $C_{1}$ and $C_{2}$ may be selected as desired to simplify eq 2.62. For the special cases to be introduced below, the derivation and/or selection of the quantities $a, b, C_{1}$ and $C_{2}$ is shown in Appendix A.3 (see also [25]). 
It is useful to note that, in the case of similarity, eq 2.62 is invariant under the transformation $F=\lambda^{2} F^{+}$and $\eta=\lambda \eta^{+}$. The common requirement of $F\left(\eta_{t i p}\right)=$ $F^{+}\left(\eta_{\text {tip }}^{+}\right)=0$ for problems involving an advancing tip permits the determination of $F_{\eta^{+}}^{+}\left(\eta_{t i p}^{+}\right)$from eq 2.62 , namely

$$
F_{\eta^{+}}^{+}\left(\eta_{t i p}^{+}\right)=\frac{b \eta_{t i p}^{+}}{2 C_{1} C_{2}^{2}}
$$

Due to the added degree of freedom introduced by $\lambda, \eta_{t i p}^{+}$may be conveniently set equal to 1 . Eq 2.63 then specifies the slope at the tip. Now, knowing both $F^{+}$and $F_{\eta^{+}}^{+}$at the tip permits the use of a single step backwards Runge-Kutta method, when numerical solution is necessary. The parameter $\lambda$, and hence $F$ and $\eta$, may then be determined by the integral mass balance or the applicable boundary condition.

It is remarkable to observe from these equations that the slope condition at the tip is given by the equation itself. Though the magnitudes of the nonlinear terms $2 F_{\eta^{+}}^{+2}$ and $\eta^{+} F_{\eta^{+}}^{+}$are maximum at the tip, the linear terms contain $F^{+}$which is approaching zero there. As a consequence, $F_{\eta^{+}}^{+}$divides out and the result is a linear system near and at the tip requiring only satisfaction of $F^{+}(1)=0$.

The invariant similarity equations for the three flow scenarios are presented below with their respective boundary conditions choosing $\eta_{t i p}^{+}=1$.

\subsubsection{Capillary Rise (Constant Height)}

This is the problem of an initially flat interface $(B o \sim \infty)$ in the $x-y$ plane. Gravity, acting in the negative $z$-direction, is suddenly "turned off" and the flow proceeds up the corner in the positive $z$-direction due to capillary forces-a process comparable to the phenomena depicted in Figure 1.2. For this problem $a=0, b=-1 / 2$, and $C_{1}=1, C_{2}=2^{-1 / 2}$ are selected. The choice of $a$ and $b$ result from an assumption of constant meniscus height at $z=0$, or rather, $h(0, \tau)=1$, which leads to $F(0)=1$ for the transformed problem.

The condition $F(0)=1$ is not obvious as the appropriate choice for this 'capillary rise' problem. Though a condition of this type has been used previously [17], it has not been demonstrated that in a time-dependent flow the condition $F(0)=1$ is established at $\eta=0$. The validity of this boundary condition will be discussed in conjunction with the presentation of experimental data acquired to this purpose. An analytic means for determining the constant height for several simple cell crosssections is provided in a following Section 2.6.8. [Note that $h=F, \eta=z(2 \tau)^{-1 / 2}$, 
$\mathcal{L}=\eta_{\text {tip }}(2 \tau)^{1 / 2}$.] The invariant eq 2.62 becomes

$$
F^{+} F_{\eta^{+} \eta^{+}}^{+}+2 F_{\eta^{+}}^{+2}+\eta^{+} F_{\eta^{+}}^{+}=0
$$

subject to

$$
F^{+}(1)=0 \quad F_{\eta^{+}}^{+}(1)=-\frac{1}{2}
$$

Numerical solution to the above system gives $F^{+}(0)=0.345 \ldots$ from which $\lambda$ may be determined in order to satisfy $F(0)=1$. Quantities of practical interest, namely, the location and slope of the interface at the tip, and the slope of the interface at $\eta=0$ may be determined as

$$
\begin{gathered}
\eta_{t i p}=\lambda=\left(F^{+}(0)\right)^{-1 / 2}=1.702 \ldots \\
F_{\eta}\left(\eta_{t i p}\right)=-\lambda / 2=-0.851 \ldots \\
F_{\eta}(0)=\lambda F_{\eta^{+}}^{+}(0)=-0.349 \ldots
\end{gathered}
$$

The numerical solution for $F^{+}\left(\eta^{+}\right)$is shown in Figure 2.7 .

A first order asymptotic solution near the tip, where $F^{+} \rightarrow 0$ and $\eta^{+} \rightarrow 1$, gives

$$
F^{+}=\frac{1}{2}\left(1-\eta^{+}\right)
$$

and so $\eta_{t i p}=2^{1 / 2}$ and $F_{\eta}(0)=F_{\eta}\left(\eta_{t i p}\right)=-2^{-1 / 2}$. It is interesting to note that this solution, though applicable to the tip region only, also gives $F(0)=h(0)=1$, that it satisfies $F_{\eta^{+} \eta^{+}}^{+}=0$ for all $\eta^{+}$, and that $\eta_{t i p}$ is reduced by approximately $17 \%$ when compared to $\eta_{t i p}$ determined by the full solution of eq 2.64. The tip solution of eq 2.66 is sketched in Figure 2.7.

\subsubsection{Spreading Drop (Constant Volume)}

This is the case for a drop of volume $H^{3}$ being instantaneously placed in an interior corner. The drop spreads in both the positive and negative $z$-directions. Symmetry is maintained across the $x-y$ plane at $z=0$ and the height of the drop at $z=0$ decreases as the fluid is pumped along the corner. A conservation of mass integral gives $a=-1 / 5, b=-2 / 5$, and $C_{1}=\left(\kappa^{2} / 5\right)^{1 / 5}, C_{2}=\left(5^{2} \kappa\right)^{-1 / 5}$ are selected, where $\kappa=\epsilon / 2 F_{A}$. [Note that for this case $h=\kappa^{2 / 5}(5 \tau)^{-1 / 5} F, \eta=\kappa^{-1 / 5} z(5 \tau)^{-2 / 5}$, and $\mathcal{L}=\kappa^{1 / 5} \eta_{t i p}(5 \tau)^{2 / 5}$.]

The governing invariant eq 2.62 for this problem is

$$
F^{+} F_{\eta^{+} \eta^{+}}^{+}+2 F_{\eta^{+}}^{+2}+2 \eta^{+} F_{\eta^{+}}^{+}+F^{+}=0
$$


subject to

$$
F^{+}(1)=0 \quad F_{\eta^{+}}^{+}(1)=-1
$$

for which an analytic solution exists

$$
F^{+}=\frac{1}{2}\left(1-\eta^{+2}\right)
$$

$\lambda$ may be determined via the integral mass balance and quantities of interest may be computed,

$$
\begin{gathered}
\eta_{t i p}=\lambda=\left(\int_{0}^{1} F^{+2} d \eta^{+}\right)^{-1 / 5}=\left(\frac{15}{2}\right)^{1 / 5}=1.496 \ldots \\
F_{\eta}\left(\eta_{t i p}\right)=-\left(\frac{15}{2}\right)^{1 / 5}=-1.496 \ldots \\
F(0)=\frac{\eta_{t i p}^{2}}{2}=\frac{1}{2}\left(\frac{15}{2}\right)^{2 / 5}=1.119 \ldots
\end{gathered}
$$

Eq 2.68 represented in terms of $h(z, \tau)$ is

$$
h=\frac{1}{2} \lambda^{2} \kappa^{2 / 5}(5 \tau)^{-1 / 5}\left(1-\eta^{2} / \lambda^{2}\right)
$$

where $0 \leq \eta \leq \lambda$. The complete result for $F^{+}\left(\eta^{+}\right)$is provided on Figure 2.7.

\subsubsection{Constant Flow}

When the flow rate $q$ at the inlet $(z=0)$ is held fixed, the amount of fluid in the corner, initially zero, increases linearly with time and the fluid profile increases both in height and length. The integral mass balance for this problem gives $a=1 / 5$, $b=-3 / 5$. Constants $C_{1}=\left(5 q^{2}\right)^{1 / 5}$ and $C_{2}=\left(5^{3} q\right)^{-1 / 5}$ are chosen. [Note that $h=$ $q^{2 / 5}(5 \tau)^{1 / 5} F, \eta=q^{-1 / 5} z(5 \tau)^{-3 / 5}$, and $\mathcal{L}=q^{1 / 5} \eta_{t i p}(5 \tau)^{3 / 5}$, where $\left.q=q^{\prime} / F_{i} W H^{2} F_{A}.\right]$

Eq 2.62 can now be written as

$$
F^{+} F_{\eta^{+} \eta^{+}}^{+}+2 F_{\eta^{+}}^{+2}+3 \eta^{+} F_{\eta^{+}}^{+}-F^{+}=0
$$

subject to

$$
F^{+}(1)=0 \quad F_{\eta^{+}}^{+}(1)=-\frac{3}{2}
$$

Numerical solution of the above gives $F^{+}(0)=1.200 \ldots$ and $F_{\eta^{+}}^{+}(0)=0.935 \ldots \lambda$ may be determined using the mass balance integral

$$
\lambda=\eta_{\text {tip }}=\left(\frac{5}{2} \int_{0}^{1} F^{+2} d \eta^{+}\right)^{-1 / 5}=0.942 \ldots
$$




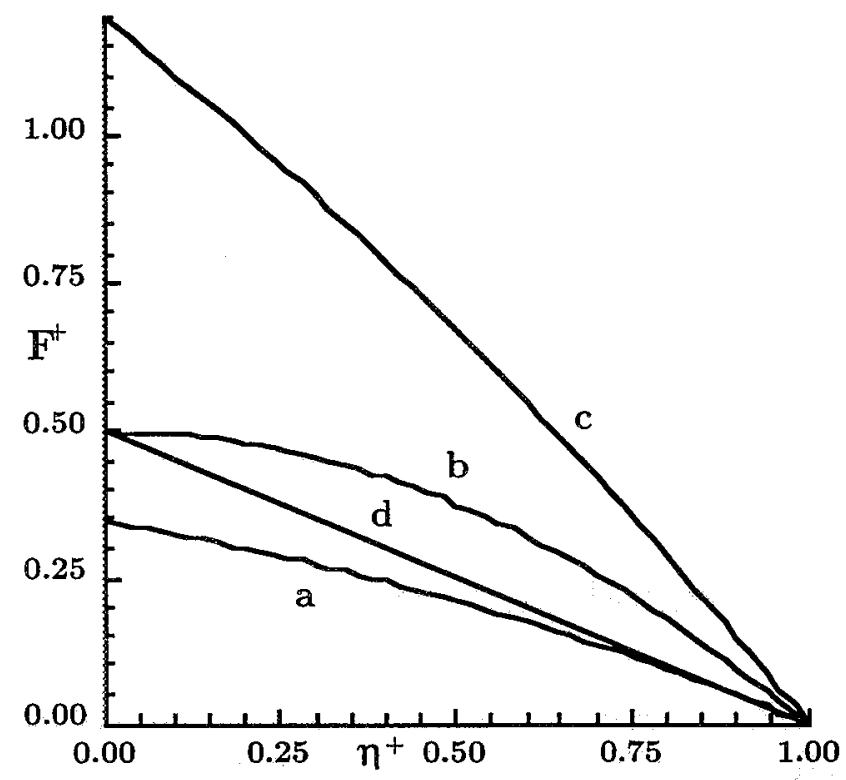

Figure 2.7: Similarity function $F^{+}\left(\eta^{+}\right)$for the (a) capillary rise, (b) spreading drop, and (c) constant flow problems. The constant slope line (d) is the approximation valid near the tip for the capillary rise problem. Note that for this solution $F^{+}(0)=1 / 2$.

\begin{tabular}{|l|l|l|l|l|}
\hline Case & $\eta_{\text {tip }}$ & $F(0)$ & $F_{\eta}(0)$ & $F_{\eta}\left(\eta_{\text {tip }}\right)$ \\
\hline a. Capillary Rise & $1.702 \ldots$ & 1 & $-0.349 \ldots$ & $-0.851 \ldots$ \\
b. Spreading Drop & $1.496 \ldots$ & $1.119 \ldots$ & 0 & $-1.496 \ldots$ \\
c. Constant Flow & $0.942 \ldots$ & $1.065 \ldots$ & $-0.881 \ldots$ & $-1.413 \ldots$ \\
d. Linear Solution $(a=0)$ & $\sqrt{2}$ & 1 & $-1 / \sqrt{2}$ & $-1 / \sqrt{2}$ \\
\hline
\end{tabular}

Table 2.4: Summarized values from the similarity solutions.

from which may be determined

$$
\begin{gathered}
F(0)=1.065 \ldots \\
F_{\eta}(0)=-0.881 \ldots \\
F_{\eta}\left(\eta_{t i p}\right)=-1.413 \ldots
\end{gathered}
$$

The result for $F^{+}\left(\eta^{+}\right)$is presented graphically in Figure 2.7.

A summary of useful values for the problems considered above is provided in Table 2.4. 


\begin{tabular}{|c|c|c|c|c|c|}
\hline$a$ & $b$ & $2 a /(1+a)$ & $F^{+}(0)$ & $F_{\eta^{+}}^{+}(0)$ & $\left(F_{\eta^{+}}^{+}(0)\right)^{-1 / 2}$ \\
\hline$-1 / 3$ & $-1 / 3$ & -1 & 0 & $\infty$ & $\infty$ \\
$-1 / 5$ & $-2 / 5$ & $-1 / 2$ & $1 / 4$ & 0 & 2 \\
0 & $-1 / 2$ & 0 & 0.345 & -0.205 & 1.702 \\
$1 / 5$ & $-3 / 5$ & $1 / 3$ & 0.400 & -0.312 & 1.581 \\
1 & -1 & 1 & $1 / 2$ & $-1 / 2$ & $\sqrt{2}$ \\
$a \gg 1$ & $-a / 2$ & 2 & 0.637 & -0.755 & 1.253 \\
\hline
\end{tabular}

Table 2.5: Selected values from the generalized similarity equation, eqs 2.73 and 2.74 .

\subsubsection{Generalized Similarity Solution}

It is instructivs to digress here to point out several characteristics of the governing similarity ODE, eq 2.62. Restricting the discussion to cases $a \geq-1 / 3$, selecting $C_{1}=1, C_{2}=|b|^{1 / 2}$, and noting the condition $b=-(1+a) / 2$, the governing eq 2.62 may be recast to give

$$
F^{+} F_{\eta^{+} \eta^{+}}^{+}+2 F_{\eta^{+}}^{+2}+\eta^{+} F_{\eta^{+}}^{+}-\frac{2 a}{1+a} F^{+}=0
$$

As described above, having introduced $\lambda$, the boundary conditions can be specified at the tip,

$$
F^{+}(1)=0 \quad F_{\eta^{+}}^{+}(1)=-1 / 2
$$

Solutions to eq 2.73 above for several values of $a$ are plotted on Figure 2.8 and the significant numerical results are tabulated in Table 2.5. The cases of capillary rise, the spreading drop, and the constant flow condition are represented by the curves $a=0,-1 / 5$, and $1 / 5$, respectively, for this particular selection of $C_{1}$ and $C_{2}$. The solution $F^{+}=\left(1-\eta^{+}\right) / 2$ is the asymptote for all solutions to eq 2.73 at the tip. Note that the leading order tip solution, $F^{+}=\left(1-\eta^{+}\right) / 2$, found for the case $a=0$ (eq 2.66) is valid for any $a$. For $a=1$ the linear solution holds, not only at the tip, but throughout $\eta^{+}$. Furthermore, the sole coefficient in eq 2.73-multiplying the last term of the left hand side-is only a slowly varying function of $a$ : for $a>0$ it is bounded between 0 and 2, and for all $a$ the coefficient is bounded by -2 and 2 . (Solutions for $a<-1 / 3$ produce curves which do not intersect the positive $F^{+}$-axis.) Consequently, for $a \approx 1$ the solution $F^{+}\left(\eta^{+}\right)$is nearly linear. 


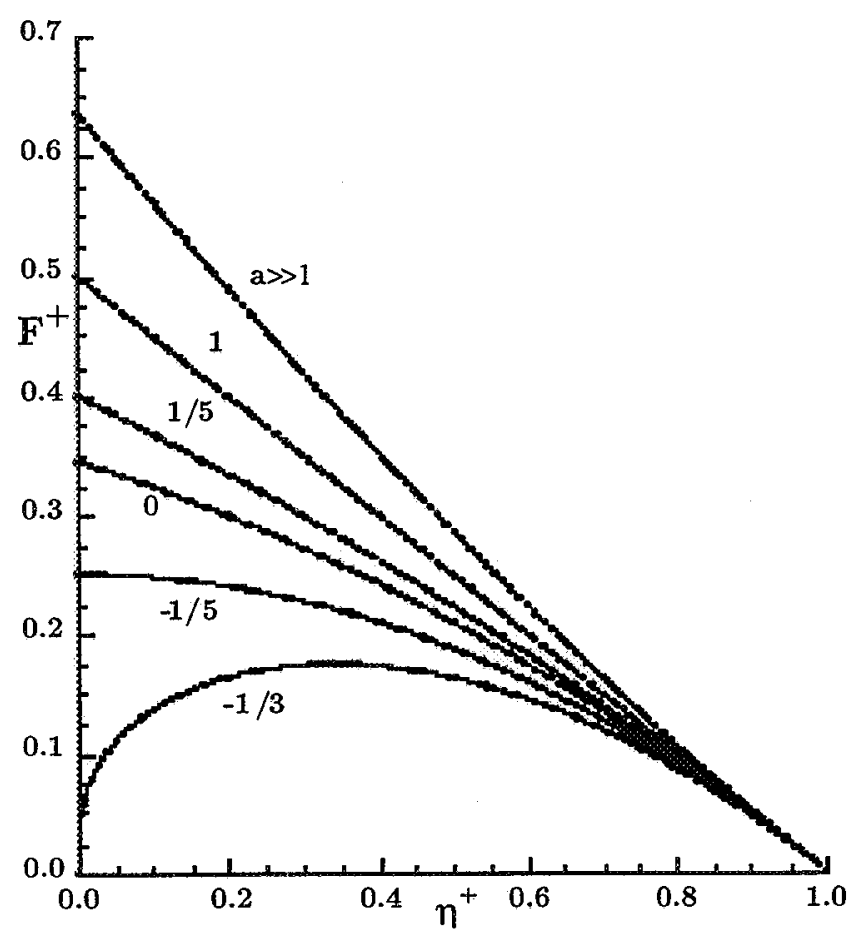

Figure 2.8: Similarity function $F^{+}\left(\eta^{+}\right)$for $a \geq-1 / 3$.

\subsubsection{Inclusion of Gravity in $x$-direction}

If gravity along the $x$-direction, $g_{x}$, is incorporated into the analysis it can be shown that the governing partial differential equation is given by

$$
h_{\tau}=2 h_{z}^{2}\left(1+2 B o_{H} h^{2}\right)+h h_{z z}\left(1+B o_{H} h^{2}\right)
$$

where $B o_{H}=f \rho g H^{2} / \sigma$ is the Bond number based on surface curvature (via $f$ ) and characteristic height $H$, and with $g$-positive acting in the negative $x$-direction (see Appendix A.4). By transforming this equation in like manner as for the similarity solutions above one obtains

$$
F F_{\eta \eta}\left(1+B o_{H} C_{1}^{2} \tau^{2 a} F^{2}\right)+2 F_{\eta}^{2}\left(1+2 B o_{H} C_{1}^{2} \tau^{2 a} F^{2}\right)-\frac{\tau^{-1-a-2 b}}{C_{1} C_{2}^{2}}\left[a F+b \eta F_{\eta}\right]=0
$$

which permits similarity solutions only when $a=0, b=-1 / 2$. This means that only solutions of the type determined for the case of capillary rise are possible. For this choice the governing equation becomes

$$
F F_{\eta \eta}\left(1+B o_{H} F^{2}\right)+2 F_{\eta}^{2}\left(1+2 B o_{H} F^{2}\right)+\eta F_{\eta}=0
$$

with $h=F, \eta=z(2 \tau)^{-1 / 2}$, and $\mathcal{L}=\eta_{\text {tip }}(2 \tau)^{1 / 2}$, subject to boundary conditions

$$
F(0)=1 \quad F\left(\eta_{t i p}\right)=0
$$


and the integral mass balance

$$
2\left(1+B o_{H}\right) F_{\eta}(0)+\int_{0}^{\eta_{t i p}} F^{2} d \eta=0
$$

Since, in general, eq 2.75 assumes that surface tension effects dominate the flow $\left(\rho g H^{2} \tan ^{2} \alpha / \sigma \ll 1\right)$, for the solution to be valid along the entire corner, it can be shown that the range of $B o_{H}$ is restricted by the condition $f^{-1} B o_{H} \tan ^{2} \alpha \ll 1$. In addition, the appropriateness of the boundary condition $F(0)=1$ is unsubstantiated. Regardless, $F$ is solved numerically for several values of $B o_{H}$ and the results are presented graphically in Figure 2.9. Solutions where $g$ is negative (acting in the positive $x$-direction) may also be determined and values for $B o_{H}>-1$ are shown. $F_{\eta}(0) \rightarrow-\infty$ as $B o_{H} \rightarrow-1$. Figure 2.9 illustrates the significant effect of negative $g$ on $F_{\eta}(0)$ and the relatively modest effect on $\eta_{t i p},{ }^{7}$ The numerical values for both may be correlated with $B o_{H}$ to produce

$$
\begin{aligned}
& \eta_{t i p}=1.702\left(1+0.286 B o_{H}\right)^{1 / 2} \\
& F_{\eta}(0)=-0.349 \frac{\left(1+0.7 B o_{H}\right)^{1 / 2}}{1+B o_{H}}
\end{aligned}
$$

These relationships are accurate to $\pm 3 \%$ for the range $B o_{H} \gtrsim-1$, but should be considered practical only in the limit $f^{-1} B o_{H} \tan ^{2} \alpha \ll 1$. The case $B o_{H} \rightarrow 0$ recovers the capillary rise result of Section 2.6.1.

\subsection{6 z-Curvature Consideration}

An interesting similarity solution is possible if the effects of curvature in the $z$ direction are considered in an asymptotic analysis. In the unique limits of $\epsilon^{2} \ll$ $\epsilon^{2} f \ll 1$, it can be shown that

$$
P_{o}=-\sigma\left(\frac{1}{h f}+\epsilon^{2} f h_{z z}\right)
$$

leading to the governing partial differential equation

$$
h_{\tau}=2 h_{z}^{2}+h h_{z z}-\epsilon^{2} f\left(4 h^{2} h_{z} h_{z z z}+h^{3} h_{z z z z}\right)
$$

\footnotetext{
${ }^{7}$ For the infinite column solution inclusive of $g_{x}$, the fluid in the corner is unstable for $B o_{H}<-1$, see eq 2.58 .
} 


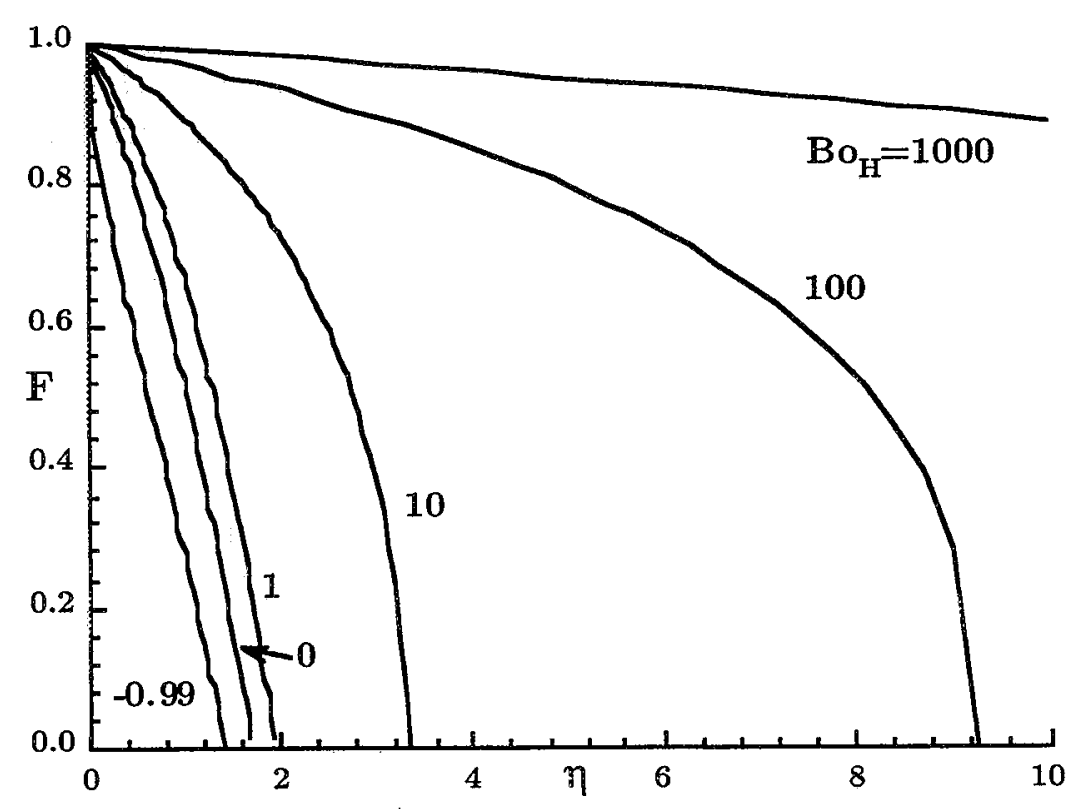

Figure 2.9: Similarity function $F(\eta)$ for the capillary rise problem for several values of $B o_{H}$.

Using the transforms of eq 2.59, similarity of the governing equation and boundary conditions may be achieved only by setting $a=-b=1(h=\tau F, \eta=z / \tau)$ and the resulting invariant system is

$$
F^{+} F_{\eta^{+} \eta^{+}}^{+}+2 F_{\eta^{+}}^{+2}+\eta^{+} F_{\eta^{+}}^{+}-F^{+}-\epsilon^{2} f F^{+2}\left(4 F_{\eta^{+}}^{+} F_{\eta^{+} \eta^{+} \eta^{+}}^{+}+F^{+} F_{\eta^{+} \eta^{+} \eta^{+} \eta^{+}}^{+}\right)=0
$$

subject to the boundary conditions $F^{+}(1)=0$ and $F_{\eta^{+}}^{+}(1)=-1 / 2$. Two additional boundary conditions are needed and $F_{\eta^{+} \eta^{+}}^{+}(1)=F_{\eta^{+} \eta^{+} \eta^{+}}^{+}(1)=0$ are likely good choices. In the limit $\epsilon^{2} f \ll 1$, a closed form asymptotic solution is possible to the leading order system: choosing $C_{1}=C_{2}=1$

$$
F^{+} F_{\eta^{+} \eta^{+}}^{+}+2 F_{\eta^{+}}^{+2}+\eta^{+} F_{\eta^{+}}^{+}-F^{+}=0
$$

subject to $F^{+}(1)=0$ and $F_{\eta^{+}}^{+}(1)=-1 / 2$. A solution is

$$
F^{+}=\frac{1}{2}(1-\eta)
$$

from which $F^{+}(0)=1 / 2$ and $F_{\eta}^{+}(\eta)=-1 / 2$. Using the integral mass balance equation $\eta_{\text {tip }}$ may be determined, but only with knowledge of $F(0)$ which raises the question as to the nature of the condition of $h(0, \tau)$. If $F(0) \equiv 1$, as was considered for the case of capillary rise, closed form expressions for $h(z, \tau)$ and $\mathcal{L}(\tau)$ are readily 
obtained. However, these solutions retain dependence on the length scales $H$ and $L$ and thus are not unique. A function $\Gamma(\epsilon)$ is introduced such that $F(0)=\Gamma(\epsilon)$. The integral continuity equation thus gives

$$
\eta_{t i p}=\lambda=[\Gamma(\epsilon)]^{1 / 2}\left[-\left.\frac{2}{3} F_{\eta^{+}}^{+}\right|_{\eta^{+}=0}\left(\int_{0}^{1} F^{+^{2}} d \eta^{+}\right)^{-1}\right]^{1 / 4}=[2 \Gamma(\epsilon)]^{1 / 2}
$$

Solving for the dimensional values for $h$ and $\mathcal{L}$ shows

$$
\begin{gathered}
h=G t \frac{\epsilon^{2} \Gamma(\epsilon)}{2}-z \frac{\epsilon[\Gamma(\epsilon)]^{1 / 2}}{2^{1 / 2}} \\
\mathcal{L}=G t \frac{\epsilon[\Gamma(\epsilon)]^{1 / 2}}{2^{1 / 2}}
\end{gathered}
$$

where

$$
G=\frac{\sigma F_{i} \sin ^{2} \alpha}{\mu f}
$$

It is interesting that if $\Gamma(\epsilon)=\epsilon^{-2}$ all $H$ and $L$ dependence vanishes, similarly as if $\epsilon=1$.

The leading order solution arises for the particular selection $a=-b=1$. This choice was necessitated by the presence of the $z$-curvature terms, which being $O\left(\epsilon^{2} f\right)$, are eventually ignored. The solution is not unique as it satisfies the homogeneous system of eq 2.37. This linear result is shown on Figure 2.8 (with $a=1$ ).

\subsubsection{Summary of Closed Form Results (dimensional)}

The analytical and numerical solutions for the cases of Sections 2.6.1-2.6.5 yield useful constants which allow the closed form determination of particular quantities of interest. These include primarily tip location and velocity (by differentiation), but flow rate and meniscus height and velocity at $z=0$ may also be determined if desired. The following are some of these results derived from the similarity solutions: $\mathcal{L}$ is the tip location, $h$ is the meniscus height and $\dot{Q}$ is flow rate at $z=0$. All quantities are dimensional in this section and the coefficient $G$ is given by eq 2.88. $F_{A}$ is given by eq 2.35 and the steady solution of eq 2.52 is provided for completeness. (Rapid and accurate estimations of the quantities listed may be made by noting $F_{A} \approx \tan \alpha$ and $F_{i} \approx 1 / 7$.) 
Steady Solution, $h=h(z)$. For the special case of $h(0)=H_{1}, h(\mathcal{L})=H_{2}(\mathcal{L}=$ const), eq 2.52 produces the dimensional results

$$
\begin{gathered}
h=H_{1}\left(1-\frac{z}{\mathcal{L}}+\left(\frac{H_{2}}{H_{1}}\right)^{3} \frac{z}{\mathcal{L}}\right)^{1 / 3} \\
\dot{Q}=\frac{F_{A} G H_{1}^{3}}{3 \mathcal{L}}\left(1-\left(\frac{H_{2}}{H_{1}}\right)^{3}\right)
\end{gathered}
$$

the latter which gives a 'maximum' capillary flow rate along a corner for these conditions.

Capillary Rise, $h(0, t)=H$. The closed form results for this problem contain $H$ which is a quantity assumed constant for the flow. The determination of $H$ for a number of simple test cell types is discussed in Section 2.6.8.

$$
\begin{gathered}
\mathcal{L}=1.702 G^{1 / 2} H^{1 / 2} t^{1 / 2} \\
\dot{Q}=0.349 F_{A} G^{1 / 2} H^{5 / 2} t^{-1 / 2}
\end{gathered}
$$

If the effect of $g$ along the $x$-axis is considered, the above are modified by the correlated coefficients for $\eta_{t i p}$ and $F_{\eta}(0)$ given in eq's 2.78 and 2.79 , which when substituted produce

$$
\begin{gathered}
\mathcal{L}=1.702\left(1+0.286 B o_{H}\right)^{1 / 2} G^{1 / 2} H^{1 / 2} t^{1 / 2} \\
\dot{Q}=0.349 \frac{\left(1+0.7 B o_{H}\right)^{1 / 2}}{1+B o_{H}} F_{A} G^{1 / 2} H^{5 / 2} t^{-1 / 2}
\end{gathered}
$$

limited to $B o_{H} \gtrsim-1$.

Spreading Drop, Volume $H^{3}$. Since an exact solution exists for this case, $h$ itself can be expressed in closed form.

$$
h=0.706 F_{A}^{-2 / 5} G^{-1 / 5} H^{6 / 5} t^{-1 / 5}\left(1-\eta^{2} / \lambda^{2}\right)
$$

where $\lambda=(15 / 2)^{1 / 5}$ and $0 \leq \eta \leq \lambda$ with

$$
\begin{gathered}
\eta=0.796 F_{A}^{1 / 5} G^{-2 / 5} H^{-3 / 5} t^{-2 / 5} z \\
\mathcal{L}=1.879 F_{A}^{-1 / 5} G^{2 / 5} H^{3 / 5} t^{2 / 5} \\
h(0, t)=0.706 F_{A}^{-2 / 5} G^{-1 / 5} H^{6 / 5} t^{-1 / 5}
\end{gathered}
$$

Constant Flow $\dot{Q}$ imposed at $z=0$.

$$
\begin{gathered}
\mathcal{L}=1.634\left(\frac{\dot{Q}}{F_{A}}\right)^{1 / 5} G^{2 / 5} t^{3 / 5} \\
h(0, t)=1.279\left(\frac{\dot{Q}}{F_{A}}\right)^{2 / 5} G^{-1 / 5} t^{1 / 5}
\end{gathered}
$$




\subsubsection{Determination of $H$, Capillary Rise}

As will be shown from experimental results, there exists a location $z_{H}$ such that the interface height $h\left(z_{H}, t\right)$ appears to rapidly rise to and remain fixed at a certain height, $H$. It is argued by Dong and Chatzis [17] that a constant pressure condition is established in the fluid at a certain $z$-location for large time. From this constant pressure condition the constant height $H$ may be evaluated. Using the elegant approach of de Lazzer and Langbein [29], $H$ is determined below for the $n$-sided regular-polygonal and rectangular cross-sectioned cylinders with sharp corners of face widths $D$ and $D_{i}$, respectively:

$$
H_{n-p o l y}=\frac{D}{2 f} \frac{\sin (\alpha+\delta)}{F_{A_{n}}}\left[1-\left(1-\frac{F_{A_{n}} \cot \pi / n}{\sin ^{2}(\alpha+\delta)}\right)^{1 / 2}\right]
$$

where $\delta=\pi / n-\theta, \alpha=\pi(n-2) / 2 n$, and

$$
H_{\text {rect }}=\frac{j D_{2}}{4 f} \frac{\sin (\alpha+\delta)}{F_{A_{n}}}\left[1-\left(1-\frac{D_{1}}{D_{2}} \frac{4 F_{A_{n}}}{j^{2} \sin ^{2}(\alpha+\delta)}\right)^{1 / 2}\right]
$$

Note for the rectangle that $D_{1} \leq D_{2}, j=1+D_{1} / D_{2}$, and $H_{n-p o l y}=H_{\text {rect }}$ for the square cross-section, $n=4, D_{1} / D_{2}=1$. $F_{A_{n}}$ is given by

$$
F_{A_{n}}=\frac{\sin ^{2} \delta}{\tan \alpha}+\sin \delta \cos \delta-\delta
$$

For the limits of $0 \leq D_{1} / D_{2} \leq 1$ it can be shown that $1 / 2 \lesssim H_{\text {rect }} f \cos \theta / D_{1} \lesssim 1$. Since ultimately $\mathcal{L} \propto H^{1 / 2}$, the effect of aspect ratio on $\mathcal{L}$ is limited to a $\sim 25 \%$ increase over that of the square cell.

The scaling introduced in Section 2.1 restricts $F_{i}$ to a narrow range of values $1 / 8 \lesssim F_{i} \leq 1 / 6$. Thus, $G \sim \sin ^{2} \alpha / f$ for all values of $\alpha$ and $\delta$. The dependence of the tip location $\mathcal{L}$ on system versus interface geometry can now be discerned. Recall that for this problem of capillary rise the tip location is $\mathcal{L} \sim(G H)^{1 / 2}$ for the $n$-sided polygon. For

$$
\frac{F_{A_{n}} \cot \pi / n}{\sin ^{2}(\alpha+\delta)} \ll 1
$$

it can be shown that $H \sim 1 / f \sin (\alpha+\delta)$ for $\alpha$ fixed. For $\alpha$ fixed it can also be shown that $G \sim 1 / f$. Thus $\mathcal{L} \sim 1 / f$ where the contribution from $H^{1 / 2} \sim f^{-1 / 2}$ is strictly geometric and is tied to the cross-flow area whereas the contribution $G^{1 / 2} \sim f^{-1 / 2}$ stems from the driving pressure gradient. In this way the effect of increasing contact angle, or equivalently the limit $\delta \rightarrow 0$, is seen to impact the tip location $\mathcal{L}$ by reducing 
the driving pressure gradient as well as the cross-sectional area for flow. This is also true for the flow rate $\dot{Q}$ since $F_{A}$ is only a weak function of $\delta$ for $\alpha$ fixed.

The reason the cross-sectional area is affected to this degree by changes in $\delta$ is due to the system pressure argument leading to the constant height boundary condition for this problem. For flow scenarios other than the capillary rise problem the effects of increasing contact angle are predominantly due to reductions in the driving pressure gradient and not to changes in cross-sectional area. This should be evident by the fact that $F_{A}$ is a banded function of $\delta, F_{A} \approx \tan \alpha$ for all $\delta$, and that $A^{\prime}=h^{\prime 2} F_{A}$. Therefore $A^{\prime}$ exhibits weak dependence on $\delta$. As a case in point, for the problem of the spreading drop (Section 2.6.2), $H$ is the cube root of the drop volume and is unrelated to any system parameters. The effects of increasing contact angle for this problem come primarily through $f$ as found in $G$ (see eq 2.95) since $F_{A}$ is a banded function in terms of $\delta$.

\subsection{Quantified Constraints}

The solutions obtained above rely on a rather large number of assumptions, the validity of which will now be investigated. The solutions themselves will be used to estimate the order of magnitude of the terms neglected in the analysis. A list of assumptions for the capillary rise problem is followed by a similar list for the problems of the spreading drop and a constant flow. Each assumption is expressed using the dimensionless numbers of eq 2.104 when possible. Otherwise dimensional quantities are used.

While the asymptotic analysis formally requires only $\epsilon^{2} \rightarrow 0$ and $\epsilon^{2} f \ll 1$, for practical application it is prudent to consider the order of magnitude of the other parameters which might appear in the problem. Thus, the constraints on $\mathcal{R}$, gravity level, and the contact line boundary condition are also considered below.

For these calculations the following terms will be used to simplify the expressions:

$$
B o_{H}=\frac{f \rho g_{i} H^{2}}{\sigma} \quad C a_{t}=\frac{\mu H}{\sigma t} \quad \mathcal{F}=\frac{f}{F_{i} \sin ^{2} \alpha}
$$

where $g_{i}$ refers to the component of $g$ in the $i$-direction. The lead constants appearing in the results are known exactly and are rounded and provided in decimal form for completeness. It is important to note that for moderate $\alpha$ in the range $\pi / 12 \lesssim \alpha \lesssim 5 \pi / 12$, and for $\delta \gtrsim \pi / 18$ (implying lower contact angles), $\mathcal{F}(\propto T)$ is approximately an $O(10)-O(100)$ quantity (see also Figure 2.6). As $\delta \rightarrow 0, \mathcal{F} \rightarrow \infty$. On the other hand, $f$ is $\sim O(1)$ for moderate $\alpha$ and $\delta \gtrsim \pi / 18$, but tends to $\sim O\left(\Omega^{-2}\right)$ and $O(\alpha / \sin \delta)$ as $\alpha$ tends to $\pi / 2$ and 0 , respectively. 
The standard assumptions of a passive overlaying gas, constant properties, and ideally sharp interior corners of infinite extent are not addressed analytically.

\subsubsection{Assumptions for Capillary Rise}

- $\epsilon^{2} \ll 1[\epsilon=H / \mathcal{L}(t)]$. This is the slender column assumption and requires

$$
0.345 C a_{t} \mathcal{F} \ll 1
$$

This requirement is not appropriate near the bulk meniscus where $\epsilon \sim O(1)$, locally.

- $\epsilon^{2} f \ll 1$. This is the requirement of slight surface curvature along the corner axis as compared to curvature perpendicular to it. It is given by

$$
0.345 f C a_{t} \mathcal{F} \ll 1
$$

This condition is difficult to satisfy as $\delta \rightarrow 0$ since $f \rightarrow \infty$ in this limit. $\mathcal{F}$ behaves similarly.

- $\mathcal{R} \ll 1$, or rather $\mathcal{R} \sim O\left(\epsilon^{2}\right)$ (see eq 2.7). In effect, this constraint is necessary in order to neglect inertial effects on the left hand side of eq 2.4. For practical low-gravity systems of typical dimensions and fluid properties it usually cannot be satisfied. It is often difficult to satisfy for normal-gravity systems as well. A more precise accounting of the constraint of low inertia is offered by $\left|\mathcal{R} w_{o_{t}} / P_{z}\right| \ll 1$, a quantitative comparison of the unsteady term to the driving pressure gradient. The convective term is found to be an order of magnitude less than the unsteady term $\left(\left|w_{o} w_{o_{z}} / w_{o_{t}}\right| \sim 0.1\right)$ and so can be ignored. This constraint can be written as

$$
\frac{h^{2} \sin ^{2} \alpha}{2 \nu t} \ll 1
$$

A worst case is when $h=H$. The presence of inertia in the problem will result in the retardation of the flow, particularly during start-up. This constraint is quite limiting for applications in low-gravity environments where $H \sim O(10 \mathrm{~cm})$ might be anticipated. However, near the tip where $h \rightarrow 0$, inertia is rightfully ignored save at very small times.

- $g_{x} \sim 0$. Negligible gravity in the $x$-direction requires

$$
B o_{H} \equiv \frac{f \rho g_{x} H^{2}}{\sigma} \ll 1
$$


- $g_{z} \sim 0$. Gravitational effects parallel to the corner axis ( $z$-direction) are negligible when

$$
B o_{H \mathcal{L}} \equiv \frac{f \rho g_{z} H \mathcal{L}}{\sigma}=1.702 B o_{H}\left(C a_{t} \mathcal{F}\right)^{-1 / 2} \ll 1
$$

The impact of $g_{z}$ increases with time as $\sim t^{1 / 2}$.

- $h(0, t)=1$, constant height assumed at $z=0$ for all $t$. This initial condition was selected based on experimental observations. Its validity needs be verified experimentally and will be addressed in Chapter 3 .

- $\theta=$ const. The solution for $w_{o}$ may be decomposed into portions perpendicular and parallel to the contact line. The component perpendicular to the contact line may be used in turn to determine the local Capillary number $\left(C a_{c l}\right)$, the magnitude of which may be used to estimate departures from the constant, static contact angle assumption. Capillary numbers of sufficient size signal the dynamic wetting phenomena leading to a dyramic apparent contact angle $\left(\theta_{d}\right)$ effect which can decrease (increase) the effective curvature (contact angle) of the free surface and so reduce the pressure gradient driving the flow. For advancing and receding contact lines, the correlation of Hoffman [30] may be used

$$
\theta_{d}=4.54\left(B+C a_{c l}\right)^{0.353} \quad \text { (radians) }
$$

where

$$
B=\left(\frac{\theta_{\text {stat }}}{4.54}\right)^{2.83}
$$

provided $B+C a_{c l} \geq 0$. The constant $B$ incorporates the effects of partial wetting. Firstly, $C a_{c l}$ is determined using $\theta=\theta_{\text {stat }}$. This value of $C a_{c l}$ is then substituted into eq 2.110 and $\theta_{d}$ is estimated. Solutions producing significant changes between $\theta_{\text {stat }}$ and $\theta_{d}$ thus determined should be reconsidered in light of dynamic contact angle effects.

The dimensional velocity of the contact line $V_{c l}$ is determined from

$$
V_{c l}=\left(h_{t}-\left\langle w_{o}\right\rangle h_{z}\right)\left(1+h_{z}^{2}\right)^{-1 / 2}
$$

Using this form and solving for $C a_{c l}=\mu V_{c l} / \sigma$ gives

$$
C a_{c l}=h_{\eta}^{2} C a_{t}\left(1-\frac{\eta}{2 h_{\eta}}\right)\left(1+h_{\eta}^{2} C a_{t} \mathcal{F}\right)^{-1 / 2}
$$

Since $h$ for this problem was determined numerically, $h_{\eta}$ must be evaluated. However, $h_{\eta}$ is bounded such that $-0.851 \leq h_{\eta} \leq-0.349$. If the constraint $\epsilon^{2} \ll 1$ is satisfied using eq 2.105 , and provided $\mathcal{F} \lesssim O(1),{ }^{8}$ eq 2.112 solved at

\footnotetext{
${ }^{8}$ This will likely satisfy the constraint $f \epsilon^{2} \ll 1$ as well.
} 
the tip (the worst case) gives

$$
C a_{c l} \doteq 1.448 C a_{t}
$$

which can be used to determine $\theta_{d}$ from eq 2.110 .

A quick and worst case estimation of dynamic contact angle effects may be made on the tip velocity prediction using

$$
\frac{V_{t i p}\left(\theta_{\text {stat }}\right)}{V_{\text {tip }}\left(\theta_{d}\right)}-1=\left[\left(\frac{F_{i}}{f}\right)_{\theta_{\text {stat }}}\left(\frac{f}{F_{i}}\right)_{\theta_{d}}\right]^{1 / 2}-1
$$

which gives the upper limit in terms of percent error due to $\theta_{d}$. The error decreases with increasing $t$ through the effect of $C a_{t} \propto 1 / t$ on $C a_{c l}$ and thus $\theta_{d}$. It is also worthy of note that for fixed $\alpha, f$ is more sensitive to changes in $\theta_{d}$ than is $F_{i}$. For instance, for $\alpha=\pi / 4$ a change of $\theta_{d}$ from 0 to $\pi / 6$ yields an increase in $F_{i}$ of $10 \%$ while $f$ increases $85 \%$. As $\delta \rightarrow 0, f \rightarrow \infty$, while $F_{i}$ cannot fluctuate more than $25 \%\left(1 / 8 \lesssim F_{i} \leq 1 / 6\right)$. Therefore the principal influence of $\theta_{d}$ is not through $F_{i}$, but through $f$ originating in the pressure scale, $P^{\prime} \sim \sigma / h^{\prime} f$.

\subsubsection{Assumptions for Spreading Drop}

For these calculations $C a_{t}, \mathcal{F}$, and $B o_{H}$ are the same as defined above and $H$ is determined from the drop volume $\left(H^{3}\right)$. In addition, $\lambda=\eta_{t i p}$ and is given by eq 2.69 as determined by the exact solution to the similarity equation. Thus, $0 \leq \eta \leq \lambda$. Since $F_{A} \sim \tan \alpha$, in the limits $\alpha \rightarrow \pi / 2$, or $\alpha \rightarrow 0$, then $F_{A} \sim(\pi / 2-\alpha)^{-1}$, or $F_{A} \sim \alpha$, respectively (see Figure 2.3).

- $\epsilon^{2} \ll 1[\epsilon=h(z, t) / \mathcal{L}(t)]$ is the slender column assumption.

$$
0.141 F_{A}^{-2 / 5}\left(C a_{t} \mathcal{F}\right)^{6 / 5}\left(1-\frac{\eta^{2}}{\lambda^{2}}\right)^{2} \ll 1
$$

The worst case is when $\eta=0$

- $\epsilon^{2} f \ll 1$ requires slight curvature along corner axis compared to perpendicular to it,

$$
0.141 f F_{A}^{-2 / 5}\left(C a_{t} \mathcal{F}\right)^{6 / 5}\left(1-\frac{\eta^{2}}{\lambda^{2}}\right)^{2} \ll 1
$$


- $\mathcal{R} \sim O\left(\epsilon^{2}\right)$, or $\left|\mathcal{R} w_{o t} / P_{z}\right| \ll 1$ is the condition necessary to neglect inertial terms.

$$
0.5\left[\left(\frac{\rho H^{3}}{\sigma t^{2}}\right)^{2}\left(\frac{H^{2}}{\nu t}\right)^{3} \frac{f^{2} F_{i}^{3} \sin ^{3} \alpha}{F_{A}^{4}}\right]^{1 / 5}\left(1-\frac{\eta^{2}}{\lambda^{2}}\right)^{2} \ll 1
$$

This term is zero at the tip $\left(\eta_{t i p}=\lambda\right)$ and is maximum at $\eta=0$ where inertial effects are greatest.

- $g_{x} \sim 0$ is the assumption that the effect of gravity perpendicular to the corner axis ( $x$-direction) is small. This constraint can be written as

$$
B o_{h} \equiv \frac{f \rho g_{x} h^{2}}{\sigma}=0.5 B o_{H} F_{A}^{-4 / 5}\left(C a_{t} \mathcal{F}\right)^{2 / 5}\left(1-\frac{\eta^{2}}{\lambda^{2}}\right)^{2} \ll 1
$$

The effect of $g_{x}$ diminishes as the tip is approached.

- $g_{z} \sim 0$ is the assumption that the effect of gravity parallel to the corner axis ( $z$-direction) must be small,

$$
B o_{h \mathcal{L}} \equiv \frac{f \rho g_{z} h \mathcal{L}}{\sigma}=3.0 B o_{H} F_{A}^{-3 / 5}\left(C a_{t} \mathcal{F}\right)^{-1 / 5}\left(1-\frac{\eta^{2}}{\lambda^{2}}\right) \ll 1
$$

The effects of $g_{z}$ decrease as the tip is approached, but increase weakly with time as $\sim t^{1 / 5}$.

- $\theta=$ const. As for the case of capillary rise, departures from $\theta_{\text {stat }}$ may be estimated by determining $C a_{c l}$. The steps follow similarly those outlined for the capillary rise problem, only here

$$
C a_{c l}=0.42 F_{A}^{-2 / 5}\left(C a_{t}^{6} \mathcal{F}\right)^{1 / 5}\left(\frac{9 \eta^{2}}{\lambda^{2}}-1\right)\left[1+\left(\frac{2}{5} C a_{t} \mathcal{F}\right)^{2}\right]^{-1 / 2}
$$

It is interesting to note the location where $C a_{c l}=0$, namely, $\eta=\lambda / 3$. The contact line upstream of this location is receding while that downstream is advancing. When $0.16\left(C a_{t} \mathcal{F}\right)^{2} \ll 1$ the above reduces to

$$
C a_{c l}=0.42 F_{A}^{-2 / 5} C a_{t}\left(C a_{t} \mathcal{F}\right)^{1 / 5}\left(\frac{9 \eta^{2}}{\lambda^{2}}-1\right)
$$

Hoffman's correlation may be used to estimate $\theta_{d}$. 


\subsubsection{Assumptions for Constant Flow}

For this section the introduction of the length scale

$$
H \equiv\left(\frac{\mu \mathcal{F} t \dot{Q}^{2}}{\sigma F_{A}^{2}}\right)^{1 / 5}
$$

simplifies the expressions and is used as ' $H$ ' was above in $C a_{t}, B o_{H}$, etc. This value for $H$ is time dependent and increases with $t^{1 / 5}$. Note that $H$ increases with increasing viscosity and flow rate $\dot{Q}$.

- $\epsilon^{2} \ll 1[\epsilon=h(z, t) / \mathcal{L}(t)]$ is the slender column assumption. The worst case at $\eta=0$

$$
0.61\left(\frac{H^{3} F_{A}}{\dot{Q} t}\right)^{2} \ll 1
$$

Although $H$ increases with time, the length of the column increases faster and $\epsilon^{2} \propto t^{-4 / 5}$.

- $\epsilon^{2} f \ll 1$ describes slight curvature along corner axis compared to that perpendicular to it,

$$
0.61 f\left(\frac{H^{3} F_{A}}{\dot{Q} t}\right)^{2} \ll 1
$$

- $\mathcal{R} \sim O\left(\epsilon^{2}\right)$ or $\left|\mathcal{R} w_{o_{t}} / P_{z}\right| \ll 1$ is the condition necessary to neglect inertial terms. It is zero at the tip and maximum at $\eta=0$. At $\eta=0$,

$$
2.62 \frac{\sin ^{2} \alpha}{f} \frac{H^{2}}{\nu t} \ll 1
$$

Thus the constraint decays slowly with time as $t^{-3 / 5}$ due to the increasing height $H$.

- $g_{x} \sim 0$ describes the assumption that the effect of gravity perpendicular to the corner axis ( $x$-direction) must be small. This is always true at the tip but for $\eta=0$

$$
B o_{h} \equiv \frac{f \rho g_{x} h^{2}}{\sigma}=1.64 B o_{H} \ll 1
$$

Note that $B o_{h}$ increases with $t^{2 / 5}$ and $\dot{Q}^{4 / 5}$. 
- $g_{z} \sim 0$ gives the assumption that the effect of gravity parallel to the corner axis ( $z$-direction) must be small and satisfy

$$
B o_{h \mathcal{L}} \equiv \frac{f \rho g_{z} h \mathcal{L}}{\sigma}=2.09 B o_{H}\left(\frac{F_{A}}{\dot{Q}}\right)\left(\frac{\sigma}{\mu \mathcal{F}}\right) t^{2 / 5} \ll 1
$$

which shows $B o_{h \mathcal{L}}$ increases with $t^{4 / 5}$ and $\dot{Q}^{3 / 5}$.

- $\theta=$ const. The presence of $\theta_{d}$ effects may be estimated similarly as for the previous cases. For the entire domain,

$$
C a_{c l}=0.48 F_{i} C a_{t} F_{\eta}^{2}\left(1-\frac{3 \eta}{2 F_{\eta}}\right)\left[1+0.48 C a_{t} \mathcal{F} F_{\eta}^{2}\right]^{-1 / 2}
$$

At the tip

$$
C a_{c l}=1.92 F_{i} C a_{t}\left[1+0.958 C a_{t} \mathcal{F}\right]^{-1 / 2}
$$

and for small $C a_{t} \mathcal{F}$ with $F_{i} \simeq 1 / 7$, the above reduces to

$$
C a_{c l}=0.27 C a_{t}
$$

$C a_{c l} \propto t^{-4 / 5}(\mu / \sigma)^{6 / 5} \dot{Q}^{2 / 5}$ and may be used with Hoffman's correlation to estimate $\theta_{d}$. The capillary number for this problem is higher in magnitude and decreases at a slower rate than for the problems of capillary rise and the spreading drop.

\subsubsection{Abbreviated Summary of Assumptions}

The assumptions analyzed above are summarized in Table 2.6. In this table, values for $\eta$ are chosen at the origin or at the tip $(\eta=\lambda)$ in order to present the maximum magnitude of the constraint under consideration. The lead constants are dropped for clarity. It should be noted that the condition to neglect inertia is $\propto 1 / \mu$ while that to satisfy $\epsilon^{2} \ll 1$ is $\propto \mu$. 


\begin{tabular}{|c|c|c|c|}
\hline Constraint & Capillary Rise & Spreading Drop & Constant Flow \\
\hline$\epsilon^{2} \ll 1$ & $C a_{t} \mathcal{F} \ll 1$ & $F_{A}^{-2 / 5}\left(C a_{t} \mathcal{F}\right)^{6 / 5} \ll 1$ & $\left(\frac{H^{3} F_{A}}{\dot{Q} t}\right)^{2} \ll 1$ \\
$\epsilon^{2} f \ll 1$ & $f C a_{t} \mathcal{F} \ll 1$ & $f F_{A}^{-2 / 5}\left(C a_{t} \mathcal{F}\right)^{6 / 5} \ll 1$ & $f\left(\frac{H^{3} F_{A}}{\dot{Q} t}\right)^{2} \ll 1$ \\
$\left|\frac{\mathcal{R} w_{o_{t}}}{P_{z}}\right| \ll 1$ & $\frac{H^{2} \sin ^{2} \alpha}{\nu t} \ll 1$ & {$\left[\left(\frac{\rho H^{3}}{\sigma t^{2}}\right)^{2}\left(\frac{H^{2}}{\nu t}\right)^{3} \frac{F_{i}^{3} \sin ^{3} \alpha}{f^{-2} F_{A}^{4}}\right]^{1 / 5} \ll 1$} & $\frac{H^{2} \sin ^{2} \alpha}{f \nu t} \ll 1$ \\
$g_{x} \sim 0$ & $B o_{H} \ll 1$ & $B o_{H} F_{A}^{-4 / 5}\left(C a_{t} \mathcal{F}\right)^{2 / 5} \ll 1$ & $B o_{H} \ll 1$ \\
$g_{z} \sim 0$ & $\operatorname{see~eq~2.119}$ & $B o_{H} F_{A}^{-3 / 5}\left(C a_{t} \mathcal{F}\right)^{-1 / 5} \ll 1$ & see eq 2.127 \\
$h(0, t)=1$ & see Chap. 3 & & \\
$\theta_{d}\left(C a_{c l}\right)$ for & $C a_{c l} \sim C a_{t}$ & $C a_{c l} \sim F_{A}^{-2 / 5} C a_{t}\left(C a_{t} \mathcal{F}\right)^{1 / 5}$ & $C a_{c l} \sim C a_{t}$ \\
$\epsilon^{2} \ll 1$ & & & \\
$C a_{t} \ll 1$ & & & \\
\hline
\end{tabular}

Table 2.6: Summary of constraints. 


\section{Chapter 3}

\section{Capillary Rise Experiments}

In this chapter a brief introduction to experimentation in a low-gravity environment is followed by a description of the capillary rise experiments and test procedures. A summary of a small portion of the test results is presented in graphical form to illustrate several global features of the flow. Digitized surface traces of the developing flow are compared with the analytical results of the preceding chapter. The initial transient and the 'start-up' flow for a sample test is discussed in light of experimental observations. A simple method for estimating tip locations at small times is suggested. For the large capillary systems which can arise in low-g environments one might expect significant inertial effects. However, it is observed that viscous forces dominate the flow in the tip region to a high degree, and that the tip flow is somewhat independent of a container length scale at small times.

Extensive experiments for the capillary rise problem were conducted which quantify the broader effects of corner angle, container size, interior surface conditions, and a wide range of fluid properties including system contact angle. These will be described in a subsequent publication. The objective for presenting only a sample of these results here is to illustrate the utility of the analytical solutions. A host of normal and low-gravity tests were also performed for the case of a spreading drop of fixed volume in an open corner (see Section 2.6.2). These tests were performed to note the impact of corner angle, contact angle, drop volume, gravity, and fluid properties on the capillary driven flow. These results will also be described in a subsequent publication. 


\subsection{Introduction to Low-g Experimentation}

Experimentation in a low-gravity (low-g) environment is unique in several respects. Container sizes can be orders of magnitude larger than their normal-gravity counterparts. The large cell size, in addition to extending the range of certain fluid parameters, also allows greater precision in container fabrication, diminishing the role of surface contamination through corner irregularity and rounỏedness, surface roughness, and other heterogeneities. In addition, larger scale capillary flows are not difficult to visualize and can be tracked over long distances. The near absence of gravity reduces the need to consider its complicating effects in analysis. Several important applications of capillary driven flows exist in the low-g environment, e.g. fluids management aboard orbiting spacecraft. It is appropriate to study such flows in a low-g environment.

A 2.2 second drop tower test facility at NASA's Lewis Research Center is used to access the low-g environment for the capillary rise tests. Though the low-g time afforded by the drop tower is limited, the cells tested aze sized to make adequate use of the time available. The $2.2 \mathrm{~s}$ drop tower is approximately $27 \mathrm{~m}$ in height. To minimize the ill-effects of aerodynamic drag on the experiment during free fall a drag shield (a box-in-a-box) technique is employed. Gravity levels of $10^{-5} g_{o}$ are common. A detailed description of this facility may be found in [31]. The description here will be restricted to the test/drop rig apparatus.

\subsection{Description of the Experiment}

A transparent test cell is first mounted to a test stand and installed in a drop rig apparatus. Prior to release of the apparatus into free fall, a prescribed amount of fluid is injected into the cell, partly filling it. Upon release, hydrostatic forces are essentially eliminated and capillary driven flow results. The flow process is backlighted by a diffuse light source and filmed at a long working distance with a high speed cine camera at $128 \mathrm{frame} / \mathrm{sec}$. A sample of frames taken from the film records is provided as Figure 3.1 for two tests of silicone oil fluid (PDMS) in a $22.6 \mathrm{~mm}$ equilateral triangular cell. Figure $3.1 \mathrm{a}$ is for $2.0 \mathrm{cs}$ fluid at $0.2 \mathrm{~s}$ intervals while Figure $3.1 \mathrm{~b}$ is for $10.0 \mathrm{cs}$ fluid at $0.5 \mathrm{~s}$ intervals (the top cross-sectional view of these cells is that of Figure 3.2b). Quantitative data is digitized directly from the film records. Up to four test cells could be tested simultaneously.

The principle variables for the drop tests are the container face width $D$ and fluid viscosity $\mu$. Corner half-angle $\alpha$ and liquid-solid contact angle $\theta$ are also parameters 


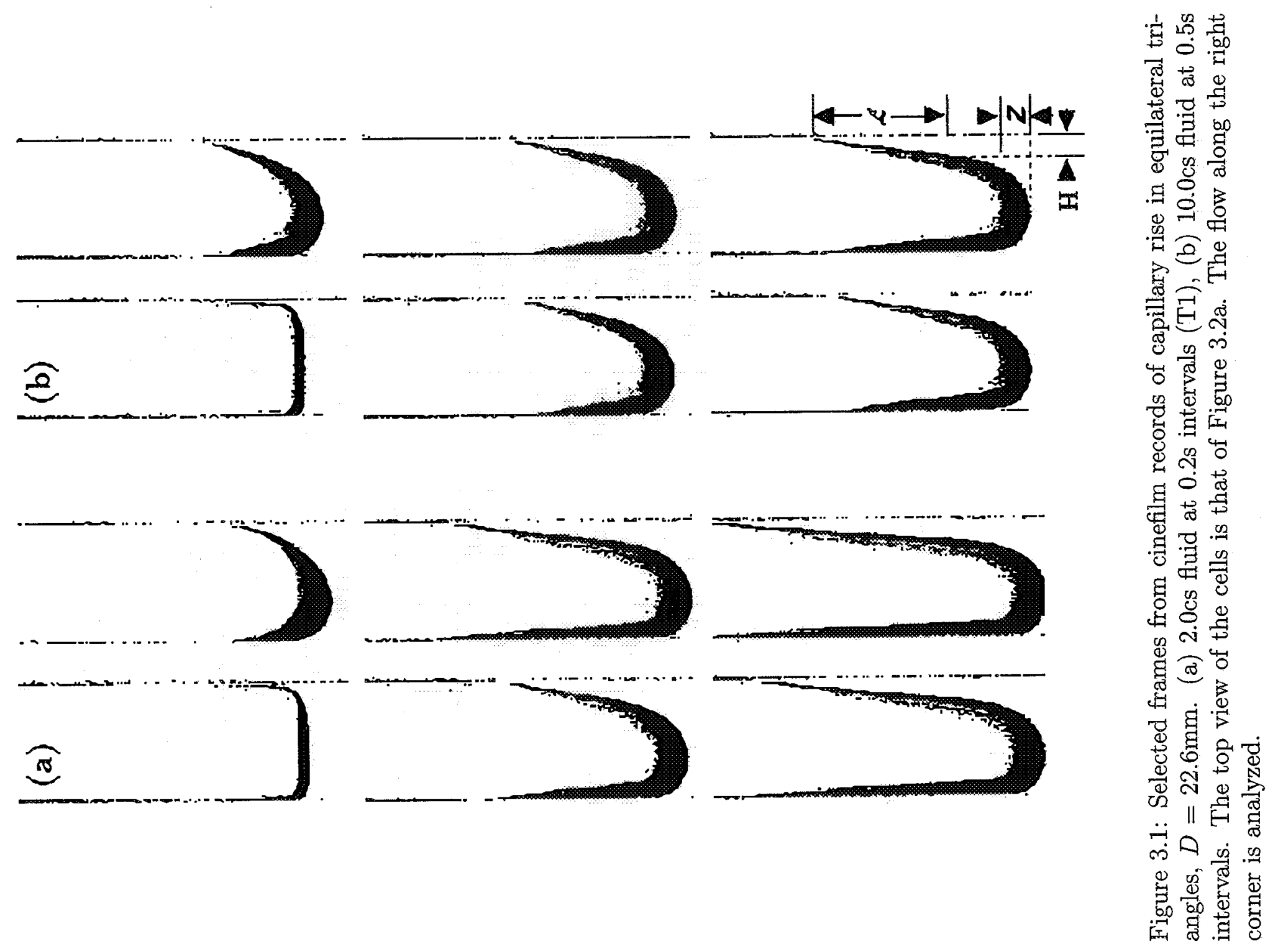




\begin{tabular}{|c|c|c|c|c|}
\hline Fluid & $\begin{array}{c}\nu \pm 2 \% \\
(\mathrm{cs})\end{array}$ & $\begin{array}{c}\rho \pm 5 \\
\left(\mathrm{~kg} / \mathrm{m}^{3}\right)\end{array}$ & $\begin{array}{c}\sigma \pm 5 \% \\
(\mathrm{~N} / \mathrm{m})\end{array}$ & $N_{D}$ \\
\hline PDMS & 0.65 & 760 & 0.0159 & 1.374 \\
PDMS & 1.0 & 816 & 0.0174 & 1.383 \\
PDMS & 2.0 & 872 & 0.0187 & 1.390 \\
PDMS & 5.0 & 913 & 0.0197 & 1.396 \\
PDMS & 10.0 & 935 & 0.0201 & 1.399 \\
PDMS & 20.0 & 949 & 0.0206 & 1.401 \\
\hline
\end{tabular}

Table 3.1. Test fluias and properties. Polydimethylsiloxane (PDMS, silicone oil) provided by Dow Corning. $\theta_{\text {stat }}=0$ on all surfaces.

\begin{tabular}{|l|l|l|l|l|c|}
\hline $\begin{array}{l}\text { Cell Cross Section } \\
\text { (section type) }\end{array}$ & Material & $\begin{array}{l}N_{D} \\
(5893 \AA)\end{array}$ & $\begin{array}{l}\mathrm{L} \\
(\mathrm{mm})\end{array}$ & $\begin{array}{l}\mathcal{D} \text { or } D_{1} \times D_{2} \\
( \pm 0.05 \mathrm{~mm})\end{array}$ & $\begin{array}{c}\alpha \\
(\mathrm{deg} .)\end{array}$ \\
\hline equil. triangle (b) & acrylic & 1.491 & 150 & 12.0 & 30 \\
equil. triangle (b) & acrylic & 1.491 & 150 & 22.6 & 30 \\
near square (c) & acrylic & 1.491 & 150 & $6.1 \times 6.7$ & 45 \\
near square (c) & acxylic & 1.491 & 150 & $12.9 \times 13.2$ & 45 \\
rectangle (c) & acrylic & 1.491 & 150 & $6.0 \times 13.0$ & 45 \\
rectarg ge (c) & acrylic & 1.493 & 350 & $6.1 \times 2 \tilde{6} .6$ & 45 \\
equil. triangle (b) & quartz & 1.459 & 60 & 5.0 & 30 \\
equil. triangle (b) & quartz & 1.459 & 60 & 10.0 & 30 \\
square (a) & quartz & 1.459 & 60 & 5.0 & 45 \\
square (a) & quartz & 1.459 & 60 & 10.0 & 45 \\
\hline
\end{tabular}

Table 3.2: Test cell data for capillary rise tests.

of interest, but in this report only systems with $\theta_{\text {stat }}=0$ will be considered. The properties of the liquids and test cells appropriate for the tests to be presented are listed in Tables 3.1 and 3.2, respectively.

As the physical condition of the corner is critical to such flows the manufacture of the test cells is also critical. Figure 3.2 displays the joining tecinique of the faces for the cells. The acrylic cells are glued and the quartz cells are fused at the joints. In order to assess fabrication variability, four reproductions of each quartz section type and two of each acrylic cell were constructed. Tolerances of the critical dimensions are included in Table 3.2. Variations in corner angle are minuscule.

The dimensions for the rectangular cells are also included in Table 3.2 where $L$ here is the total length of the cell. The majority of the tests were performed using cells of square or equilateral triangular cross sections. For both of these cell 

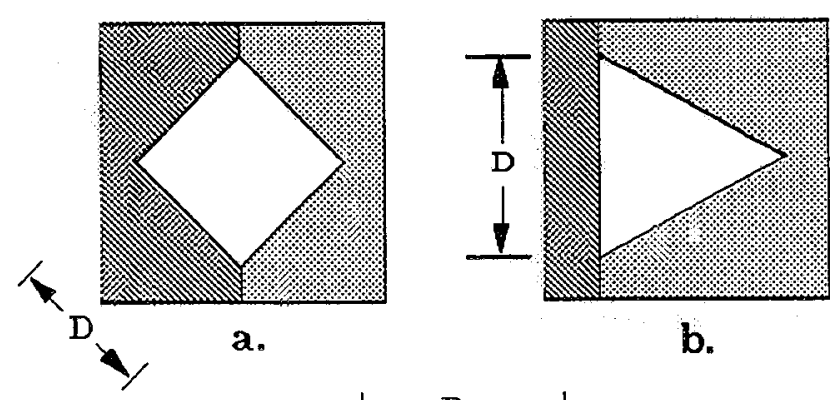

b.

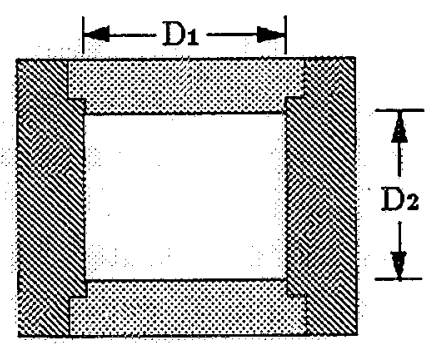

c.

Figure 3.2: Cross sections of capillary rise test cells.

configurations, the cells are fabricated such that the meniscus centerline along the corner $h(z, t)$ can be viewed in profile with a minimum of optical distortions. Figures $3.2 \mathrm{a}$ and $3.2 \mathrm{~b}$ show the cross-sections for these cell types. A ray trace analysis, described in Appendix B, which provides corrections for distortion when refractive indices are not matched, is employed for certain measurements. These corrections are never greater than $10 \%$.

Cell cleaning procedures were developed for each cell material to provide an acceptable degree of control over the surface condition. The cell preparation includes washing with a strong soap solution in hot water, rinsing thoroughly with distilled water, then rinsing with ethanol and/or methanol followed by a final distilled water rinse. The cells are allowed either to air dry or are dried in a vacuum oven. An additional acetone wash step is added for the glass cells before the ethanol rinses.

\subsection{Preliminary Measurements}

The principal measured quantities from the film records are the location of the leading tip of the fluid in the corner $\mathcal{L}(t)$, the global meniscus height $\mathcal{Z}(t)$, and the surface profile, or meniscus centerline height $h(z, t)$ (see Figure 3.1 for notation, all length measurements are in $\mathrm{mm}$ ). A sample of these results is provided and, where appli- 
cable, the dependent variable is plotted against $t^{1 / 2}$ as suggested by theory. Figures 3.3-3.7 are intended to convey the general character of noteworthy aspects of the flow using the point location measurements of $\mathcal{L}$ and $\mathcal{Z}$. A comprehensive analysis of two data sets is deferred to Section 3.4.

\subsubsection{Tip Location, $\mathcal{L}(t)$}

Figures 3.3 and 3.4 display data comparing the impact of viscosity, cell size, and corner angle on the tip location, $\mathcal{L}(t)$. Fogure 3.5 illustrates the effect of container aspect ratio for rectangular cross-sectioned cells. All tests here are performed with $\theta_{\text {stat }}=0$ in acrylic cells. The linearity of the data seems to imply the $t^{1 / 2}$ dependence of $\mathcal{L}$ for this flow which was demonstrates by Dong and Chatzis [17] to be correct at long times. As anticipatec, the tip velocity increases with decreasing viscosity and increasing cell size as observed in [17]. The tip velocity is also seen to increase for $\alpha=30^{\circ}$ compared to $\alpha=45^{\circ}$ for cells of approximately the same inscribed circle diameter. ${ }^{1}$ The solid lines are fits $\mathcal{L}=c+m t^{1 / 2}$ to the data (symbols) and exclude the initial data points for times $t \lesssim 0$.1s.

Concerning Figure 3.5, it is interesting to observe that the slope of the fits increase only modestly for a 4 -fold increase in aspect ratio. This is due to the relative insensitivity of $H$ to $D_{1} / D_{2}$ as could be demonstrated analytically by eq 2.101 .

The measurements for these figures are made using a film motion analyzer in manual mode without image processing. Multiple drop tests are made to note the repeatability of the measurements in regards to cleaning technique, fill technique, etc. The majority of tests show repeatability ${ }^{2}$ to within $\pm 6 \%$ when comparing fitting parameter coefficients for the different runs and $\pm 3 \%$ is common. With this level of precision it is easy to quantify differences in flow behavior from vessel to vessel, and even from corner to corner of the same vessel. Concerning the latter, as regards the tip location, asymmetric flows are detected between different corners of the same vessel. These differences are repeatable (again to within $\pm 6 \%$ ) and are linked to discrepancies in the fabrication quality of the corners. The largest differences are observed in the tip location measurements for the rectangular cross section vessels, yet are always less than $10 \%$.

It is important to note that when the Concus-Finn condition is satisfied, regardless of the gravity level, a thin column of fluid already exists in the corner prior to the drop test [2]. This preexisting film makes it difficult to determine the true tip location

\footnotetext{
${ }^{1}$ Recall that the case of $\alpha=30^{\circ}$ provides the most rapid response of the fluid (see Section 2.5).

${ }^{2}$ This is true when the number of duplicate tests was statistically significant.
} 


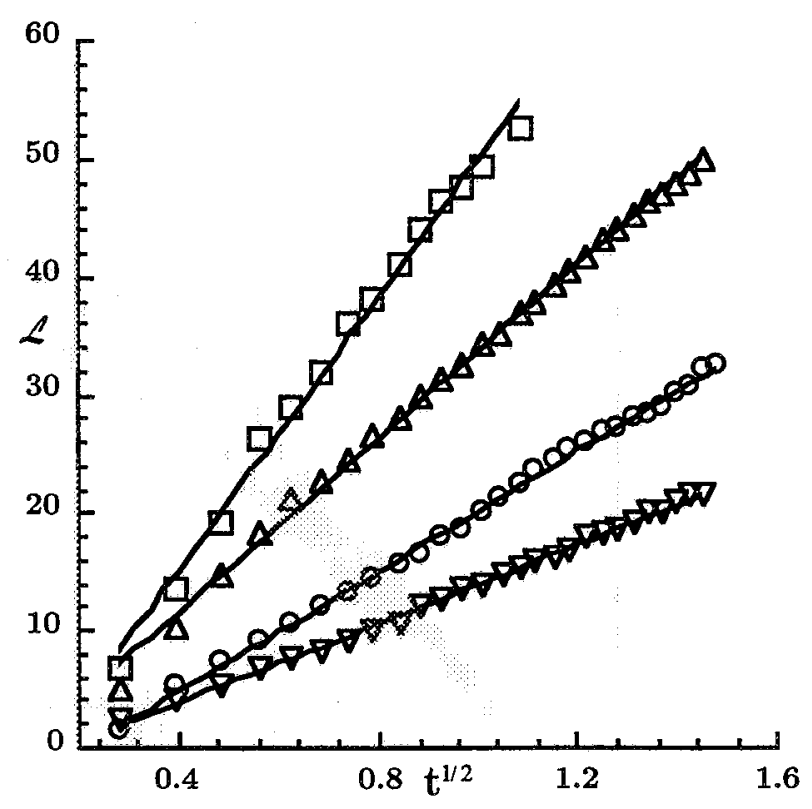

Figure 3.3: $\mathcal{L}(\mathrm{mm})$ as a function of $t^{1 / 2}\left(\mathrm{~s}^{1 / 2}\right)$ in acrylic triangle, $D=12.0 \mathrm{~mm}$, for fluids of different viscosity. $\square 1.0, \triangle 2.0, \bigcirc 5.0$, and $\nabla 10.0 \mathrm{cs}$.

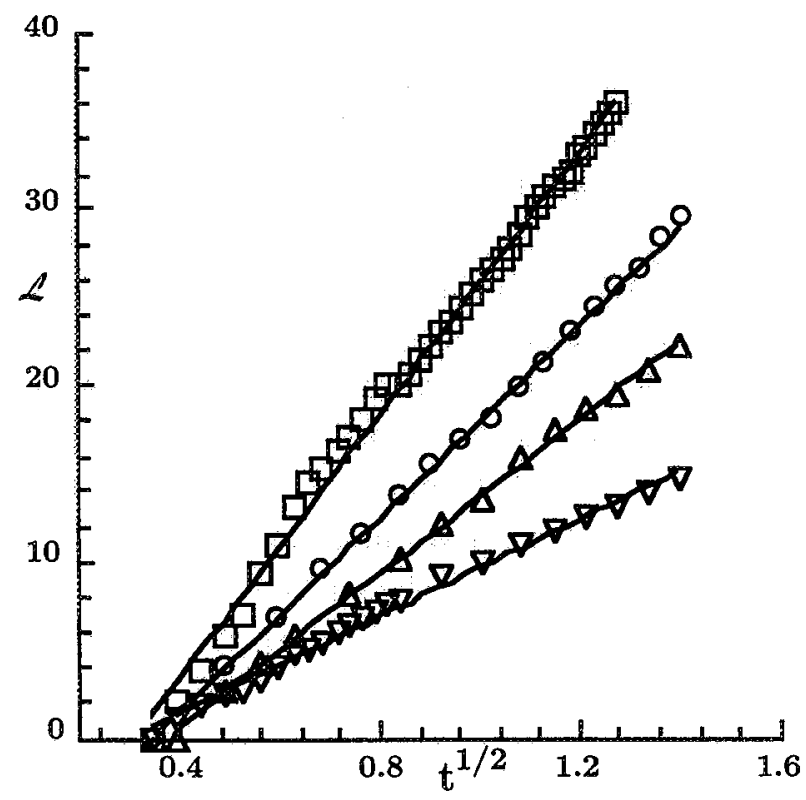

Figure 3.4: $\mathcal{L}(\mathrm{mm})$ as a function of $t^{1 / 2}\left(\mathrm{~s}^{1 / 2}\right)$ for a fluid of $\nu=5 \mathrm{cs}$ in an acrylic cell showing the effect of cell size and corner angle. $\square D=22.6 \mathrm{~mm}$ triangle, $\bigcirc 12.0$ triangle, $\triangle 12.9$ near square, and $\nabla 6.1$ near square. 


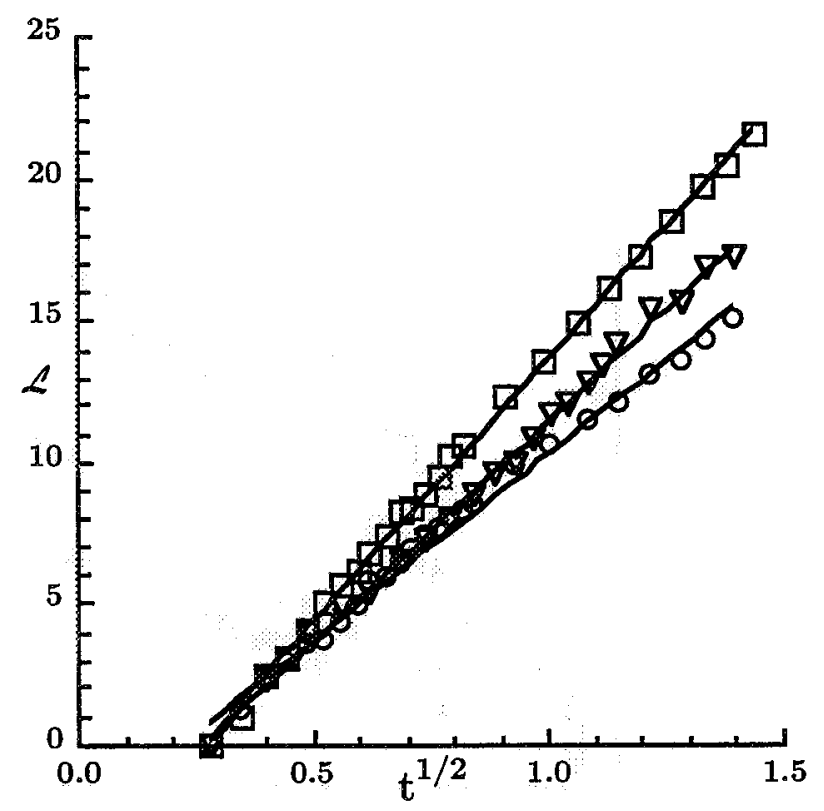

Figure 3.5: $\mathcal{L}(\mathrm{mm})$ as a function of $t^{1 / 2}\left(\mathrm{~s}^{1 / 2}\right)$ in acrylic rectangular cells for a fluid of $\nu=5.0 \mathrm{cs}$ showing the effect of aspect ratio. $\bigcirc D_{1} \times D_{2}=6.1 \times 6.7 \mathrm{~mm}, \nabla 6.0 \times 13.0$, and $\square 6.1 \times 25.6$.

of the meniscus during the early stage of the drop tests. An apparent $\mathcal{L}(t)$ was thus measured at the onset of the flow by extrapolating the apparently linear meniscus profile near the tip to the vertex of the corner. The infinite rise of the fluid in the corner is based on a continuum approach and of course an infinite corner. Provided the corner is long enough, the effects of corner imperfection, finite molecular size, Van der Waals forces, and even evaporation will prevent such an unbounded height. As the fluid column rises during the drop, upper portions of the corners are reached where the film is not observed and data are taken in these regions without any need for extrapolation. A smooth transition between data measurement techniques is achieved as can be seen from the data. It is arguable that flow in the preexisting film is so dominated by viscosity, and the slope of the surface so slight, that its presence for a portion of the bulk flow (on average $0.1 \mathrm{~s}$ of the $2.2 \mathrm{~s}$ drop time) is of little significance.

Another complication in the tip measurements is that during the latter stages of the corner flow the tip location for some of the tests (usually low viscosity fluids in the smaller test cells) becomes increasingly difficult to locate as the slope of the meniscus approaches zero. Though the rise can be viewed without mistake at high playback speeds, stop-action digitization of the tip location leads to some loss of accuracy for these cases. How much loss is uncertain. For these tests, repeat measurements of a 


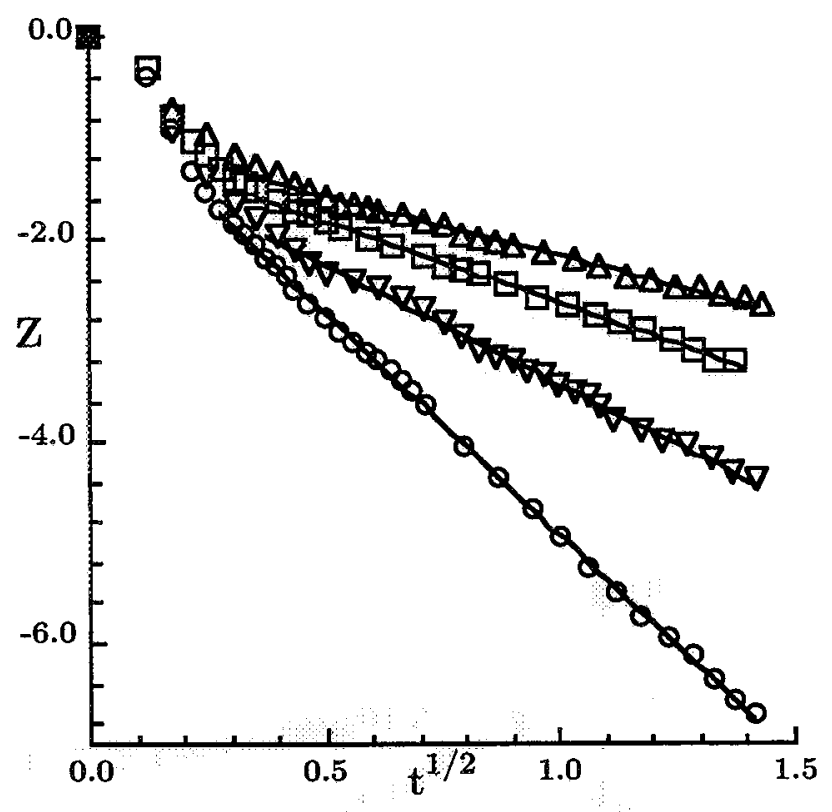

Figure 3.6: $\mathcal{Z}(\mathrm{mm})$ as a function of $t^{1 / 2}\left(\mathrm{~s}^{1 / 2}\right)$ in an acrylic triangle with $D=12.0 \mathrm{~mm}$ for fluids of various viscosity. $\bigcirc \nu=0.65 \mathrm{cs}, \nabla 2.0, \square 5.0$, and $\triangle 10.0$.

single test event show measurement repeatability ('experimenter' error) on the order of the experiment repeatability itself, namely $\pm 3-6 \%$ when comparing fit coefficients.

\subsubsection{Global Meniscus Height, $\mathcal{Z}(t)$}

Figures 3.6 and 3.7 show the decrease of $\mathcal{Z}$ with time, which is related to the drain rate of the container. As was the case in Figures 3.3 and 3.4, for the cells tested here, the flow rates and flow velocities increase with decreasing viscosity, increasing cell size [17], and decreasing corner angle for this range of $\alpha \gtrsim 30^{\circ}$. The dimensions of the cells used in Figures 3.4 and 3.7 were selected so that the radius of the inscribed circle is approximately the same for the large triangle and the large square cell. The small triangle and small square cells also share approximately the same inscribed circle radius.

\subsection{Detailed Measurements}

From the results of the point measurements presented in Figures 3.3-3.7 one might conclude that the similarity solution of Section 2.6.1, producing the $t^{1 / 2}$ dependence 


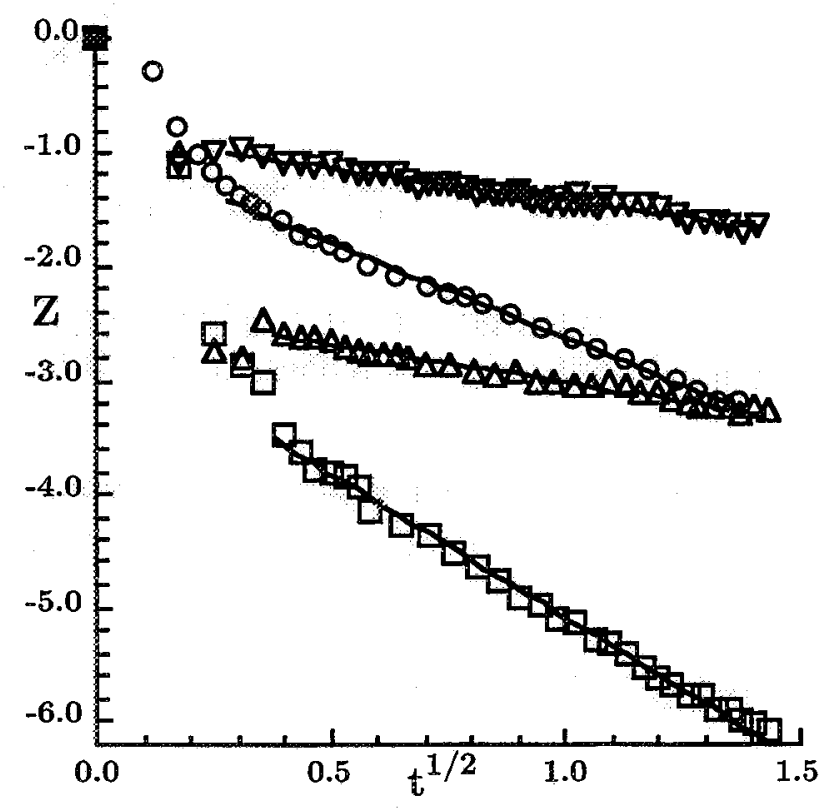

Figure 3.7: $\mathcal{Z}(\mathrm{mm})$ as a function of $t^{1 / 2}\left(\mathrm{~s}^{1 / 2}\right)$ for a fluid of $\nu=5 \mathrm{cs}$ in an acrylic cell, showing the effect of cell shape and size. $\square D=22.6 \mathrm{~mm}$ triangle, $\bigcirc 12.0$ triangle, $\triangle 12.9$ near square, and $\nabla 6.1$ near square. 


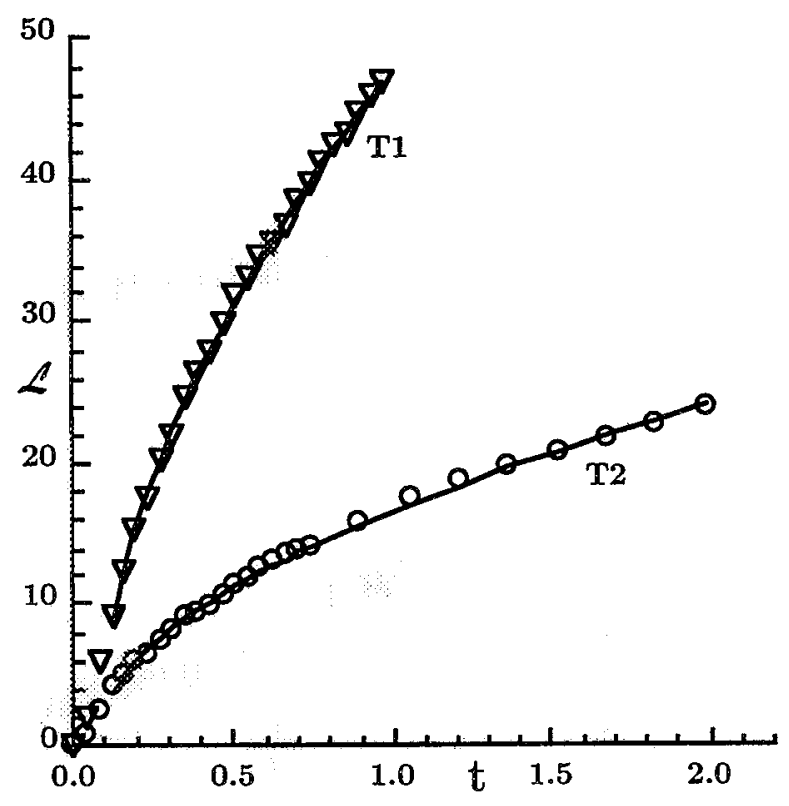

Figure 3.8: $\mathcal{L}(\mathrm{mm})$ as a function of $t(\mathrm{~s})$ in acrylic triangular cells with $c+m\left(t-t_{f}\right)^{1 / 2}$ fits. $\nabla \nu=2.0 \mathrm{cs}, D=22.6 \mathrm{~mm}\left(\mathrm{~T} 1: c=-6.17 \mathrm{~mm}, m=54.88 \mathrm{~mm} / \mathrm{s}^{1 / 2}, t_{f}=0.041 \mathrm{~s}\right)$, $\bigcirc \nu=10.0, D=12.0\left(\mathrm{~T} 2: c=-0.43 \mathrm{~mm}, m=17.61 \mathrm{~mm} / \mathrm{s}^{1 / 2}, t_{f}=0.063 \mathrm{~s}\right)$.

for the flow, is in general correct for times $t \gtrsim 0$.1s. Such a conclusion is premature as will demonstrated below using surface profile measurements of $h(z, t)$.

The measurements from two capillary rise tests of PDMS in equilateral triangular cells will be presented and contrasted in this and the following sections. In the test denoted $\mathrm{T} 1$ the viscosity is $2.0 \mathrm{cs}$ and the face width $D=22.6 \mathrm{~mm}$. For the test denoted T2 the viscosity is $10 \mathrm{cs}$ and $D=12.0 \mathrm{~mm}$. $\mathcal{L}(t)$ and $\mathcal{Z}(t)$ are presented in Figures 3.8 and 3.9 for both tests, the data of the former being fitted by a line of the form $\mathcal{L}=c+m\left(t-t_{f}\right)^{1 / 2}$.

\subsubsection{Surface Profile, $h(z, t)$ for data set $\mathbf{T} 1$}

In Figure 3.10 are overlayed ten digitized surface traces for the corner flow of test T1. These data, and the data to be presented subsequently, are acquired using an automated image analysis system which digitizes the cine film records for selected frames and tracks the development of the meniscus in time. The grabbed images are low-pass filtered and a median threshold is selected to define the interface which is then skeletonized. Figure 3.10 suggests that there is a location $z=z_{H}$ such that the height at the location $h\left(z_{H}, t\right) \equiv H$ is fixed for sufficiently long times. In the 


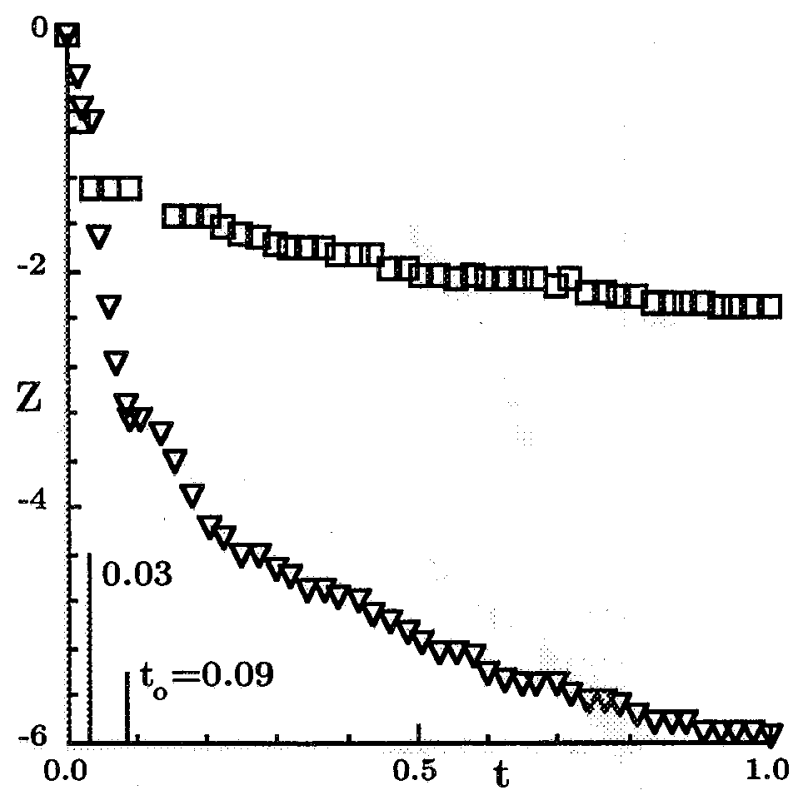

Figure 3.9: $\mathcal{Z}(\mathrm{mm})$ as a function of $t(\mathrm{~s})$ for $\mathrm{T} 1\left(\nabla, t_{o} \simeq 0.09 \mathrm{~s}\right), \mathrm{T} 2\left(\square, t_{o} \simeq 0.03 \mathrm{~s}\right)$.

figure, this location appears to occur at approximately $z_{H} \simeq 11 \mathrm{~mm}$. A magnification of the region near this point depicts the waist created by the curves passing through a narrow neighborhood near $z_{H}$, Figure $3.10 \mathrm{~b}$. Measurements of the local surface height, $h\left(z_{i}, t\right)$, at several $z$-locations, $z_{i}$, in this region are presented in Figure 3.11. The distance between each $z_{i}$ is approximately $1.5 \mathrm{~mm}$. As can be seen from the figure, depending on $z_{i}, h\left(z_{i}, t\right)$ will in general under- or overshoot $h\left(z_{H}, t\right) \equiv H$. The data of Figure 3.11 for $t>0.7 \mathrm{~s}$ are replotted and fit with linear coefficients on Figure 3.12. This figure reveals that there indeed exists a $z=z_{H}$ such that $h\left(z_{H}, t\right)=H_{e x p}=$ const $\left(H_{e x p}=x_{w a l l}-x_{H}\right)$. Interpolation between the smallest positive and negative slopes provides the empirically determined $z=z_{H}$ and $H_{\exp }$. The interpolated $z_{H}$ is between the hollow and solid triangles on the figure. In subsequent figures, the origin is moved to $z=z_{H}$. This location then defines the apparent origin where the constant height boundary condition of the asymptotic analysis can be applied. Downstream of this location $h$ is always increasing towards $H$ while upstream $h$ is decreasing everywhere to this same level. Since only limited time was available for these tests the long time behavior of the boundary condition at $z_{H}$ cannot truly be confirmed. However, the results of Dong and Chatzis whose experiments lasted hours offer strong evidence that the constant height ('constant pressure') condition is acceptable. $H=3.67 \mathrm{~mm}$ determined analytically as discussed in Section 2.6 .8 compares well with $H_{\text {exp }}=3.73 \pm 0.15 \mathrm{~mm}$ determined experimentally for this test, as noted on Figure 3.11. Uncertainty in the experimental measurement 

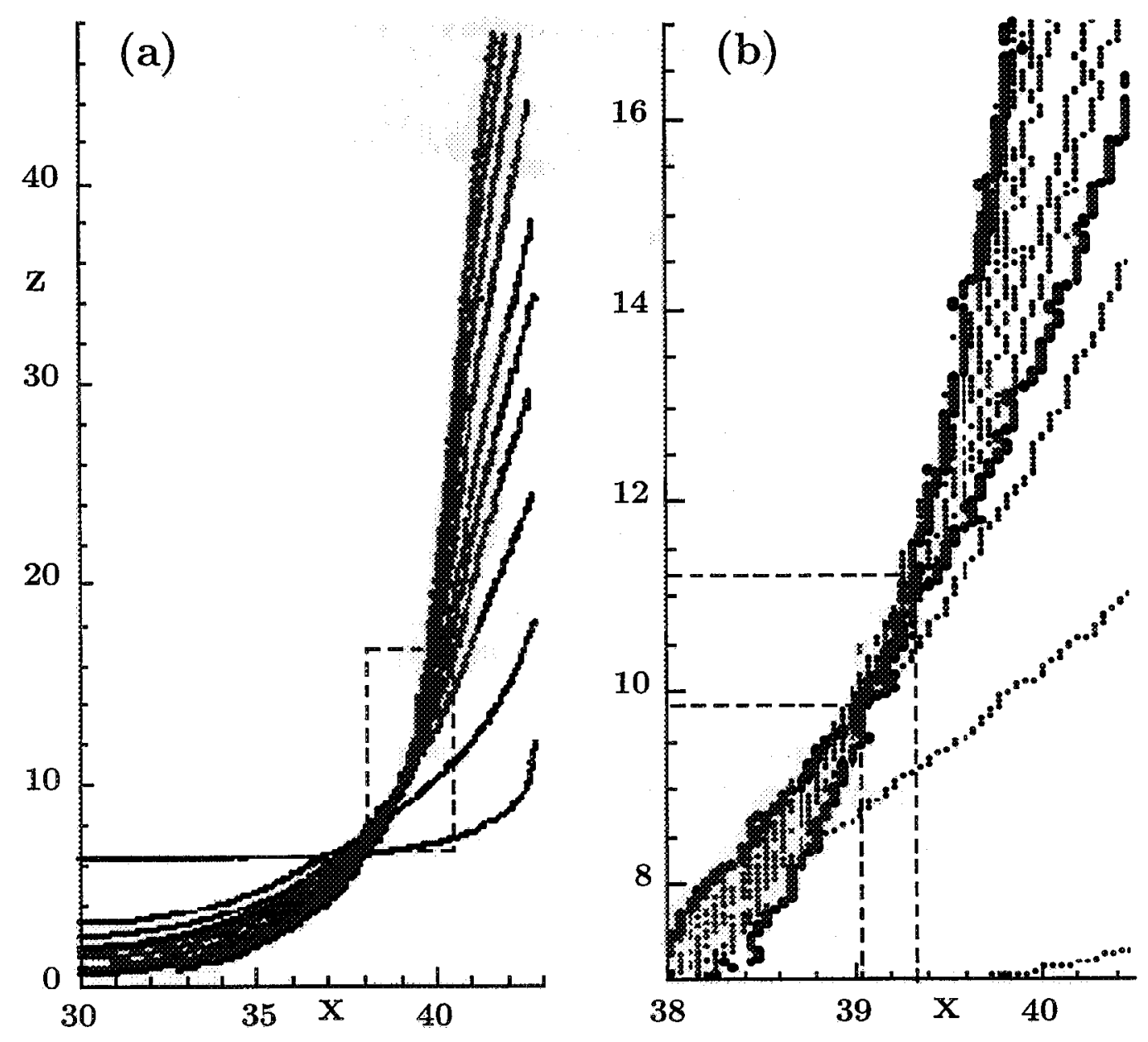

Figure 3.10: Traces of the surface height $h(z, t)$ measured along $x$ for T1. As time increases the profiles recede at the centerline $x \approx 30 \mathrm{~mm}$ and advance along the wall at $x=43.0 \mathrm{~mm}$. The surface profiles are shown for times $t=0,0.078,0.156,0.234$, $0.312,0.390,0.547,0.703,0.859$, and $1.015 \mathrm{~s}$. (a) Global profile to cell centerline, (b) enlarged view of dashed box area on (a). The coordinates $x$ and $z$ are in $\mathrm{mm}$. 


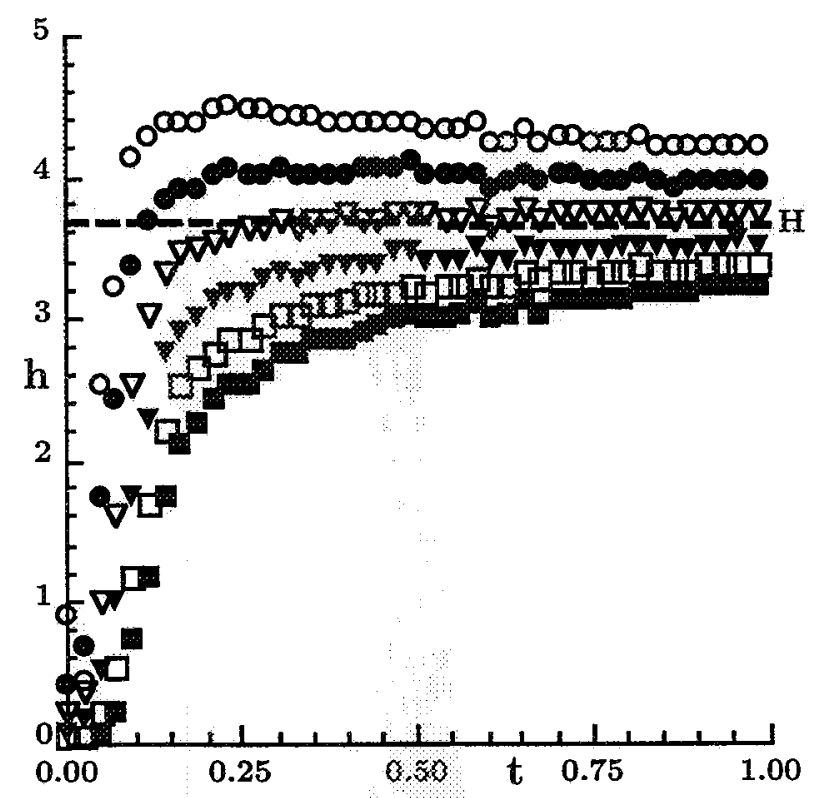

Figure 3.11: $h\left(z_{i}, t\right)(\mathrm{mm})$ as a function of $\frac{t}{2}(\mathrm{~s})$ for T1. Each data set is taken for constant $z_{i}$ with an increment of approxiriazely $1.5 \mathrm{~mm}$ between each $z_{i}$.

of $H$ is due to the optical scale factor of the measurements and the corrections for image distortion caused by refraction.

$H$ is not fully established until approximately $t_{H} \simeq 0.4 \mathrm{~s}$. The initial inertial transient $t_{o} \sim\left(\rho R_{i c}^{3} / \sigma\right)^{1 / 2}$ for the establishment of the low-g interface may be observed by the rapid decline of the meniscus $\mathcal{Z}(t)$ early during the drop test. These times are noted on Figure 3.9 along with the estimates $t_{o}=0.09$ and $t_{o}=0.03 \mathrm{~s}$ for $\mathrm{T} 1$ and T2, respectively. Note that $t_{o} \simeq 0.75\left(\rho R_{i c}^{3} / \sigma\right)^{1 / 2}$ and that the general order of $t_{o}$ agrees with the fitted time origin $t_{f}$ of Figure 3.8. Siegert et al. [32] demonstrate that the initial time response of an interface after a step reduction in gravity level may be correlated by $t_{o}=C\left(\rho R^{3} / \sigma\right)^{1 / 2}$, where $R$ is a characteristic length and $C$ is a constant, both of which depend on the container geometry. For a right circular cylinder of radius $R=R_{i c}$ they find that $C=0.413$.

The experimental values of $z_{H}$ and $t_{H}$ are used to rescale the height data; Figure 3.13 shows $h$ as a function of $z t^{-1 / 2}$ and $z\left(t-t_{H}\right)^{-1 / 2}$ where $z=z_{i}-z_{H}$. The failure of the curves to collapse when plotted against the (dimensional) similarity parameter $z t^{-1 / 2}$ suggested by theory attests to the significant effects of the start-up transients necessary to establish the constant height condition assumed instantaneous in the analysis. $H$ is achieved by $t_{H} \simeq 0.4 s$ which is selected as the time origin for the plot of Figure 3.13b. Again, sufficient time is not available to provide full agreement with the analytically determined similarity profile, which is shown on the figure. It is 


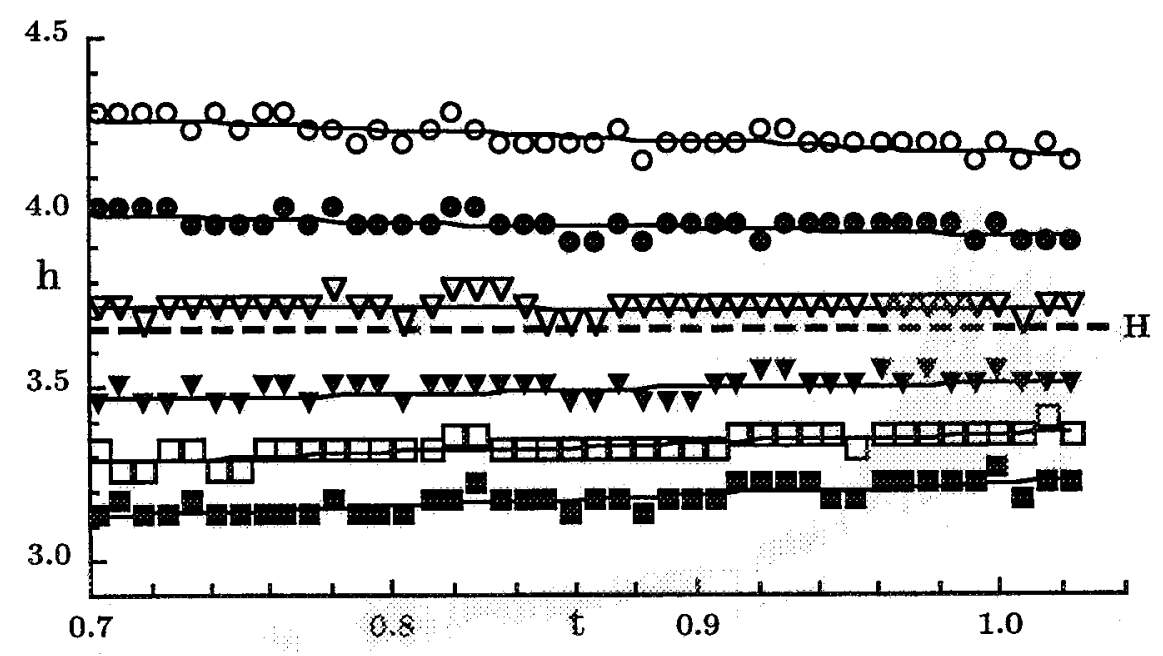

Figure 3.12: $h\left(z_{i}, t\right)(\mathrm{mm})$ as a function of $t(\mathrm{~s})$ for six fixed locations $z=z_{i}$ and $t>0.7 \mathrm{~s}$. Straight lines are fitted to the data. By interpolation, the location for which the slope is found to be zero is $z_{H}=11.24 \mathrm{~mm}$, where $H_{\text {exp }}=3.73 \pm 0.15 \mathrm{~mm}$. The theoretical value $H=3.67 \mathrm{~mm}$ is shown by the dashed line.

apparent from Figure $3.13 \mathrm{~b}$ that, after an initial overstoot, the traces are converging to the analytical result as time increases (see arrow on figure). In Figure 3.14, the time origin is shifted to $t_{o}=0.0 \mathrm{~s}_{\mathrm{s}} \mathrm{a}$ s suggested by both the inertial time scale $t_{o}$, Figure 3.9, and the fit coefficient for $\mathcal{L}(t)$, Figure 3.8. A collapse of the data is seen being especially sood near the tip, but a general collapse of the surface profiles for $0.09 \lesssim t \lesssim 0.4 \mathrm{~s}$ is also established. The similarity solution of eq 2.64 is provided for reference.

\subsubsection{Surface Profile, $h(z, t)$ for data set T2}

Figures 3.15-3.18 present the data for test T2. For this test $z_{H}=13.72 \mathrm{~mm}$ (Figures 3.15 and 3.16), and $H_{\text {exp }}$ is found to be $1.97 \pm 0.08 \mathrm{~mm}$ (Figure 3.16) compared to $H=1.94 \mathrm{~mm}$ calculated by eq 2.100 . The time at which the constant height condition is first established is found to be $t_{H} \simeq 0.4 \mathrm{~s}$. As for test $\mathrm{T} 1$, the surface traces do not collapse in the time available when plotted against $z t^{-1 / 2}$, Figure 3.17a. However, the plot of $h$ as a function of $z\left(t-t_{H}\right)^{-1 / 2}$, Figure $3.17 \mathrm{~b}$, displays nearly full agreement with the similarity result by the end of the test, $t=2.031 \mathrm{~s}$. Due to the limited duration of the test, ample time was not available to guarantee this observation for $t \gg t_{H}$. The initial overshoot and convergence of the scaled profiles toward the theoretical solution as time increases is also observed for T2 using the time origin 

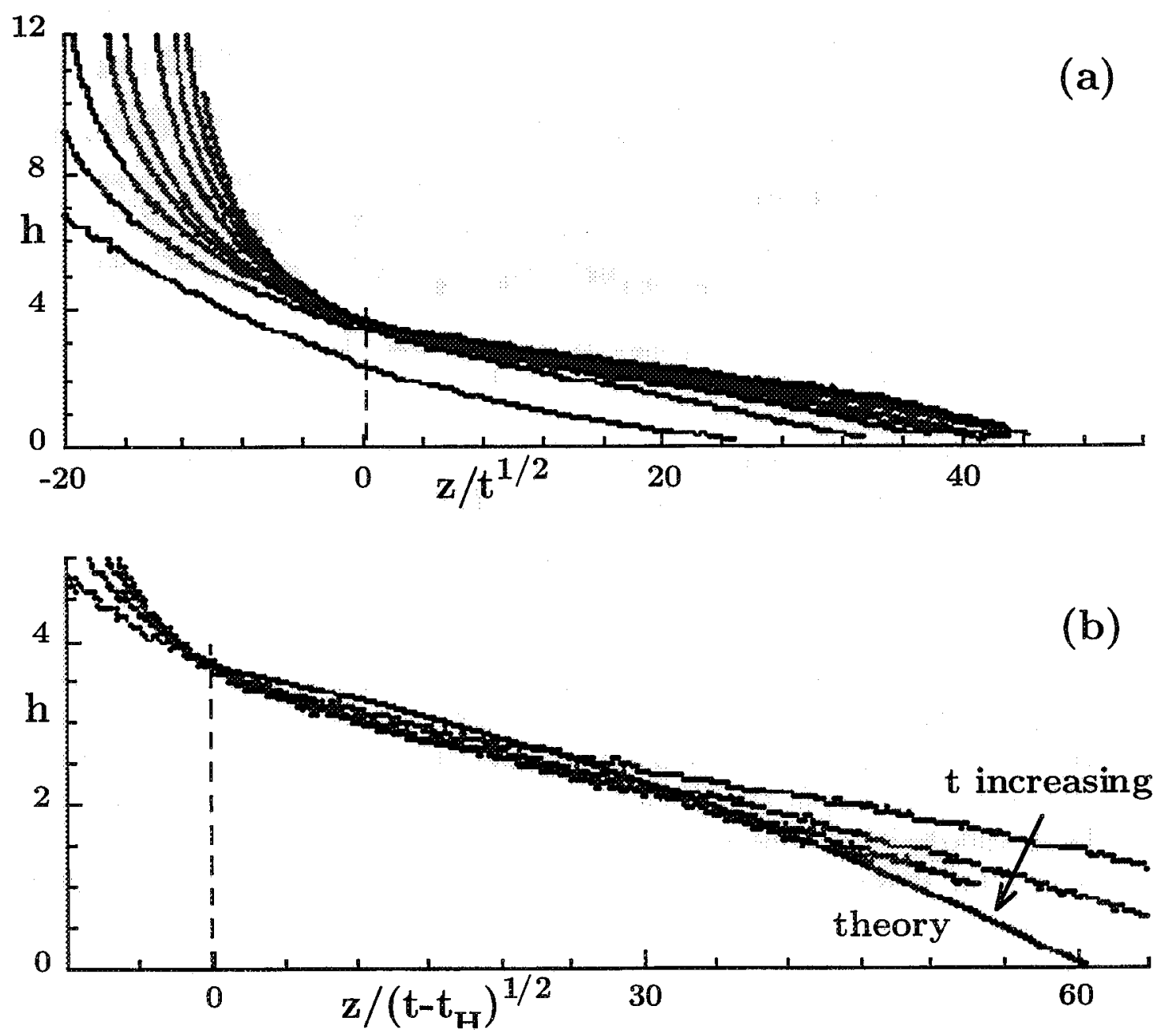

Figure 3.13: (a) $h(\mathrm{~mm})$ as a function of $z / t^{1 / 2}\left(\mathrm{~mm} / \mathrm{s}^{1 / 2}\right)$ for nine of the ten surface profiles of test T1. Trace at $t=0$ is not shown. (b) As in (a) except that the time origin is shifted by $t_{H}=0.4 \mathrm{~s}$. The last four traces are shown, the first trace is at $t=0.547 \mathrm{~s}$. The traces converge towards the theoretical curve as $t$ increases as indicated by the arrow. 


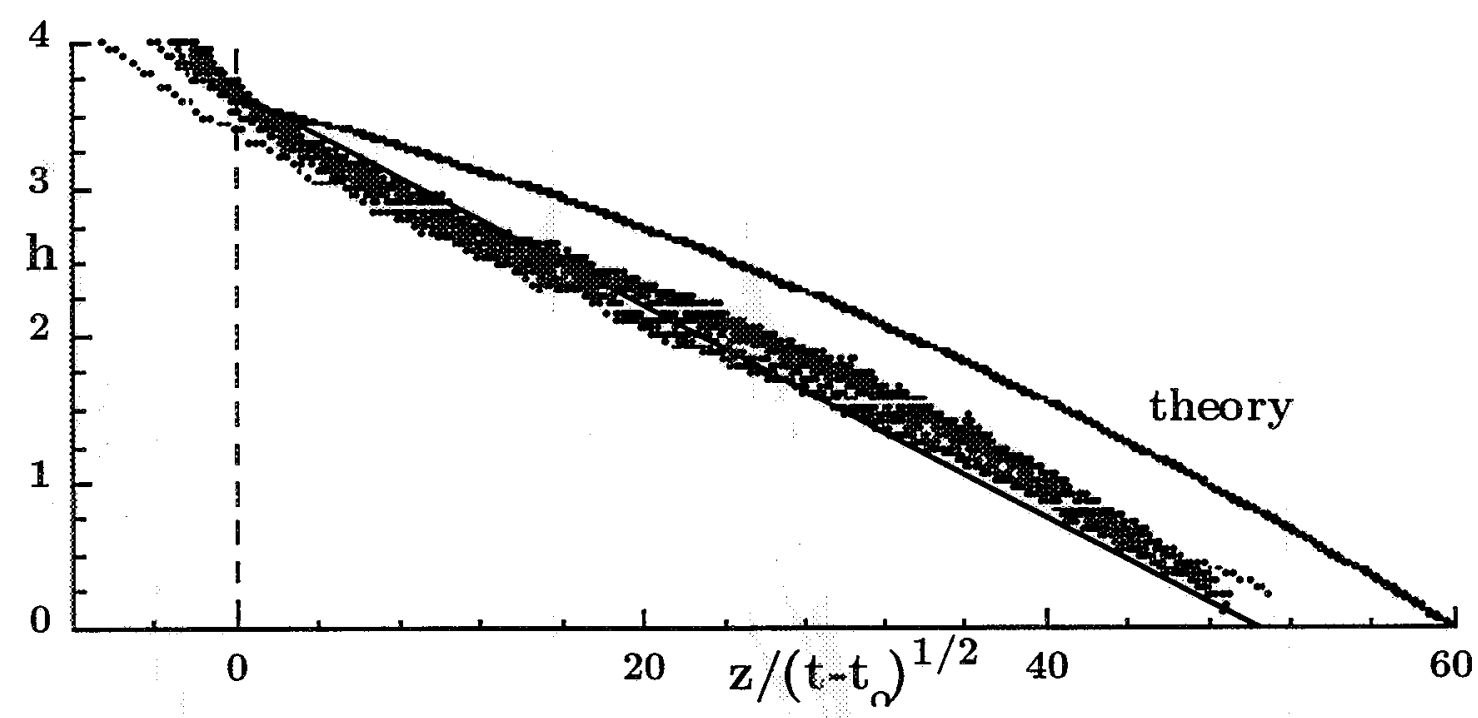

Figure 3.14: $h(\mathrm{~mm})$ as a function of $z /\left(t-t_{o}\right)^{1 / 2}\left(\mathrm{~mm} / \mathrm{s}^{1 / 2}\right)$ for $\mathrm{T} 1$ with $t_{o}=0.09 \mathrm{~s}$ providing tip collapse. Full theory provided along with linear tip solution. The first trace shown is at $t=0.156 \mathrm{~s}$.

$t_{H}$, as is indicated in Figure 3.17b. The tip location data for T2 (see Figures 3.8 and 3.9) are best fit for the time origin $t_{o}=0.03 \mathrm{~s}$ whick is employed in Figure 3.18.

\subsection{Discussion}

For cases $\mathrm{T} 1$ and $\mathrm{T} 2$, Table 3.3 lists the important time constants arising from the assumptions inherent in the asymptotic analysis (see Section 2.7.1). The Ohnesorge number $O h$ is also provided. The first two time scales are the viscous and slender body scales calculated from eqs 2.107 and 2.105 with $\alpha=30^{\circ}, \theta=0$. The third is the 'system' inertial time scale which characterizes the time required for the transition. from a gravity dominated to a surface tension dominated interface [32]. $R_{i c}^{2} / 2 \nu$ is the global viscous time scale where $R_{i c}$ is the radius of the inscribed circle for the test cell cross-section. Comparing the magnitude of these scales, it is not surprising that T2 should agree more favorably with the predictions than should T1 over such short duration tests. Considering the low values of the time constants in the table, what is surprising is that $\mathrm{T} 2$ is not fully predicted by the theory. ${ }^{3}$

\footnotetext{
${ }^{3}$ Cases $\mathrm{T} 1$ and $\mathrm{T} 2$ were selected from a family of tests conducted to explore repeatability and sensitivity to container surface condition. Within each family, whether the containers were initially
} 


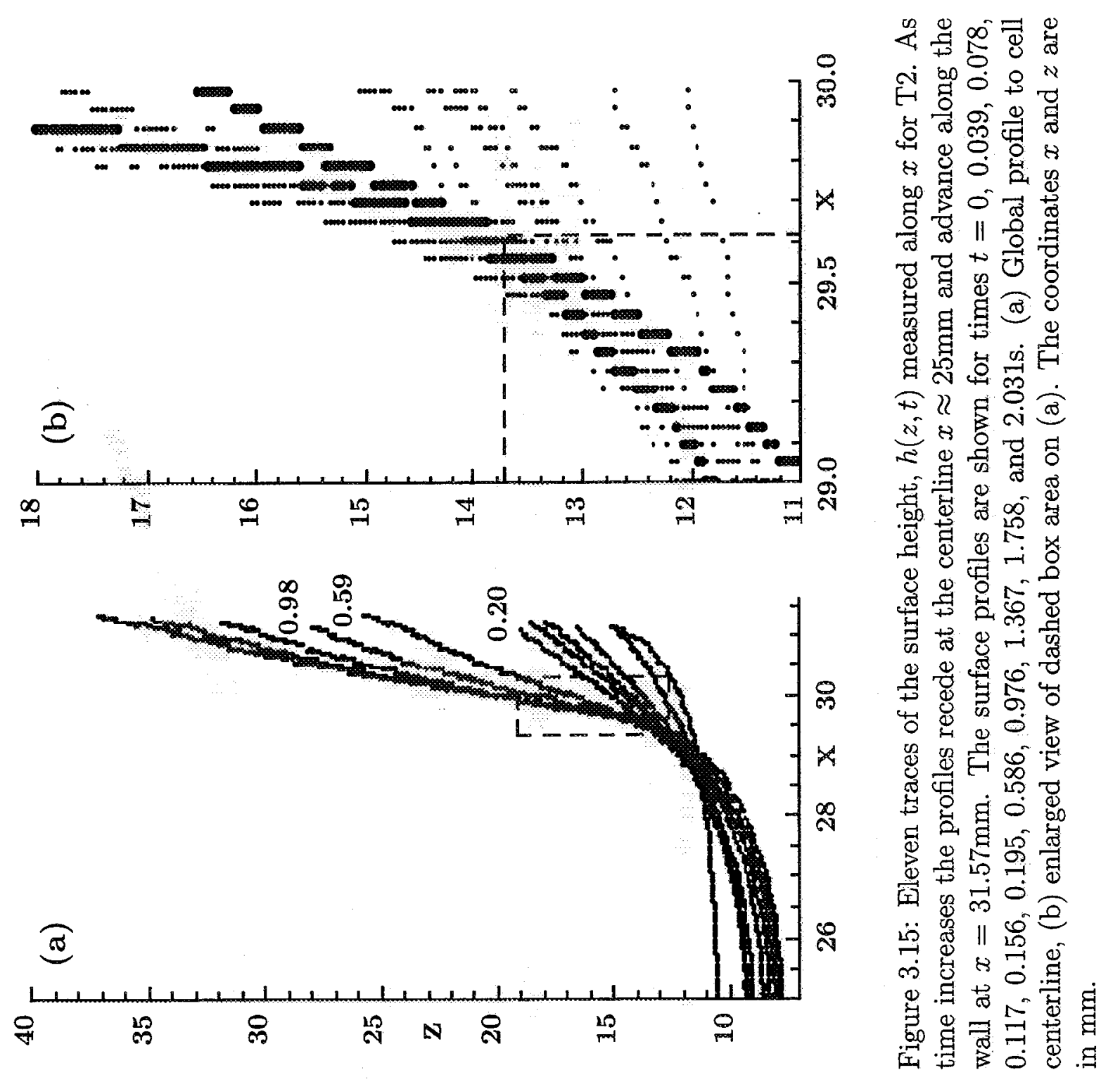




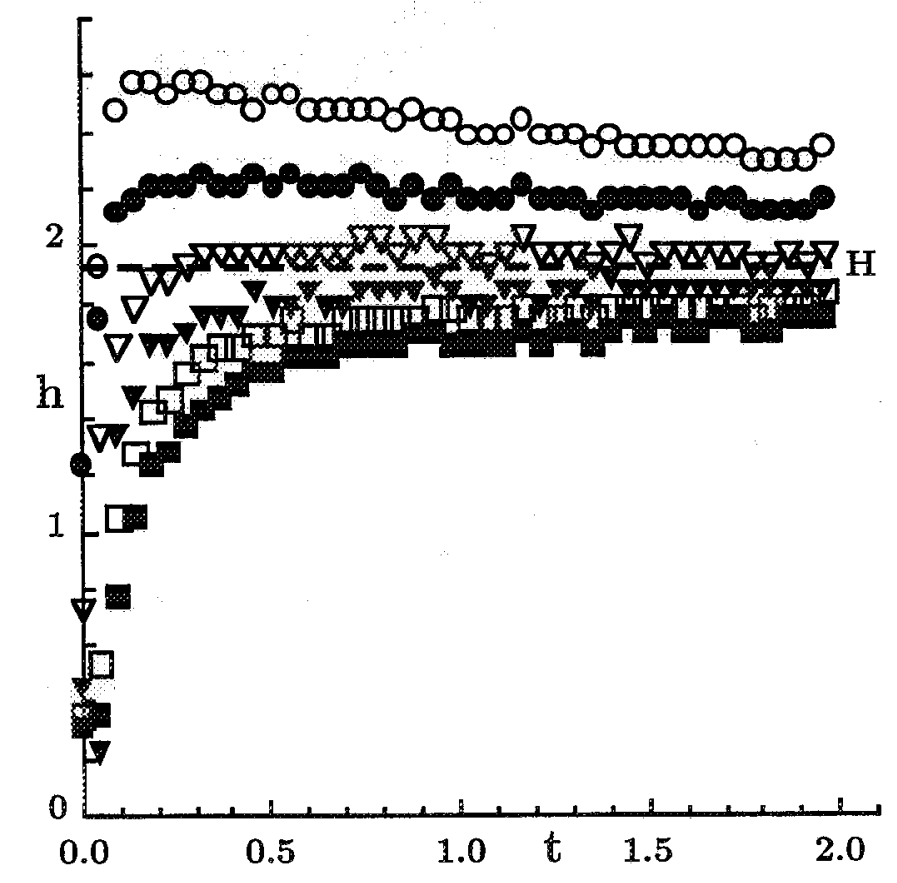

Figure 3.16: $h\left(z_{i}, t\right)(\mathrm{mm})$ as a function of $t(\mathrm{~s})$ for T2. Each data set is at constant $z_{i}$ where there is a constant increment of approximately $0.8 \mathrm{~mm}$. 

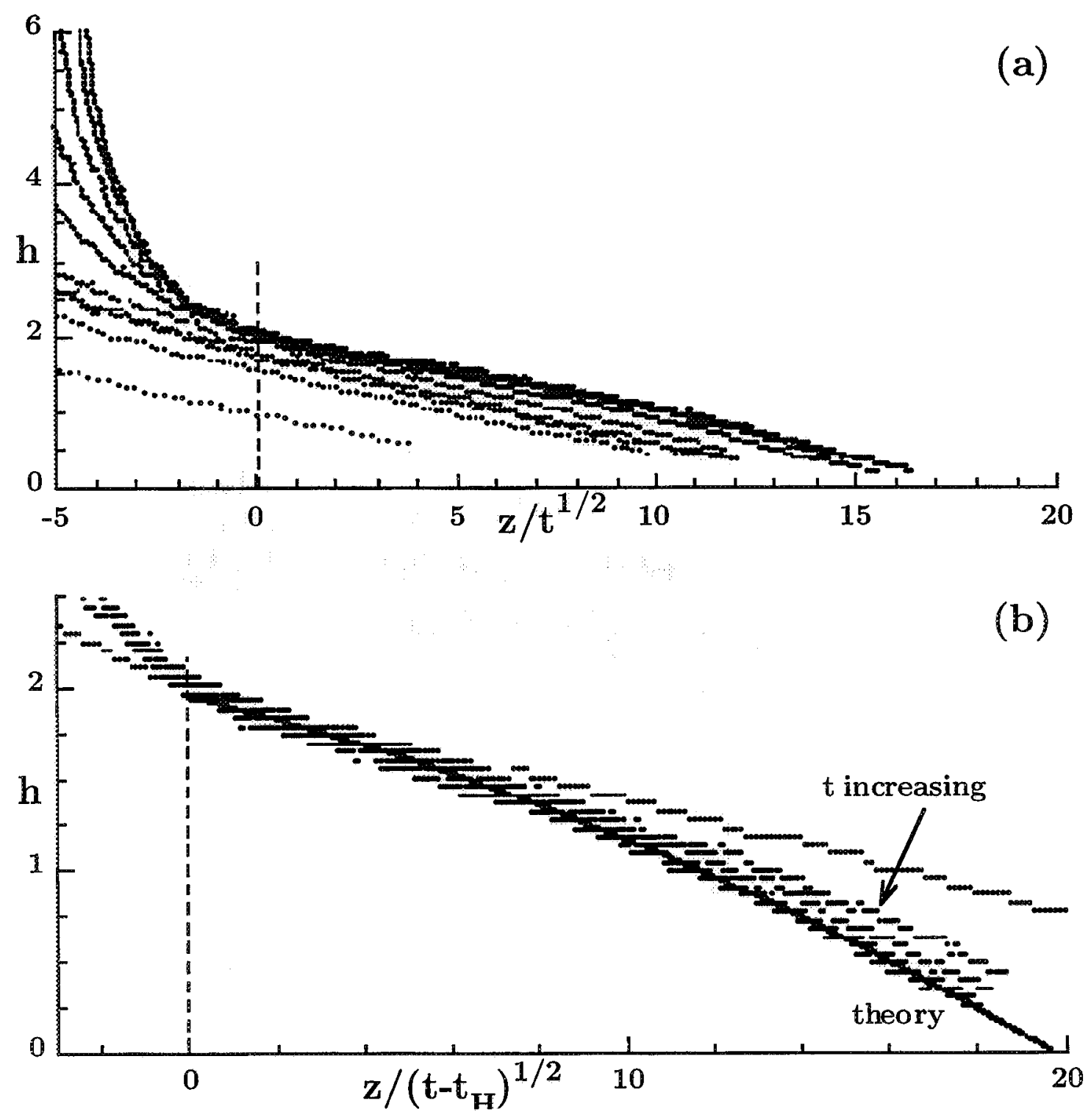

Figure 3.17: (a) $h(\mathrm{~mm})$ as a function of $z / t^{1 / 2}\left(\mathrm{~mm} / \mathrm{s}^{1 / 2}\right)$ for ten of the eleven surface profiles of test T2. Trace at $t=0$ is not shown. (b) As in (a) except that time origin is shifted by $t_{H}=0.4 \mathrm{~s}$. The last five traces are shown, the first trace is at $t=0.586 \mathrm{~s}$. The traces converge towards the theoretical curve as $t$ increases, as indicated by the arrow. 


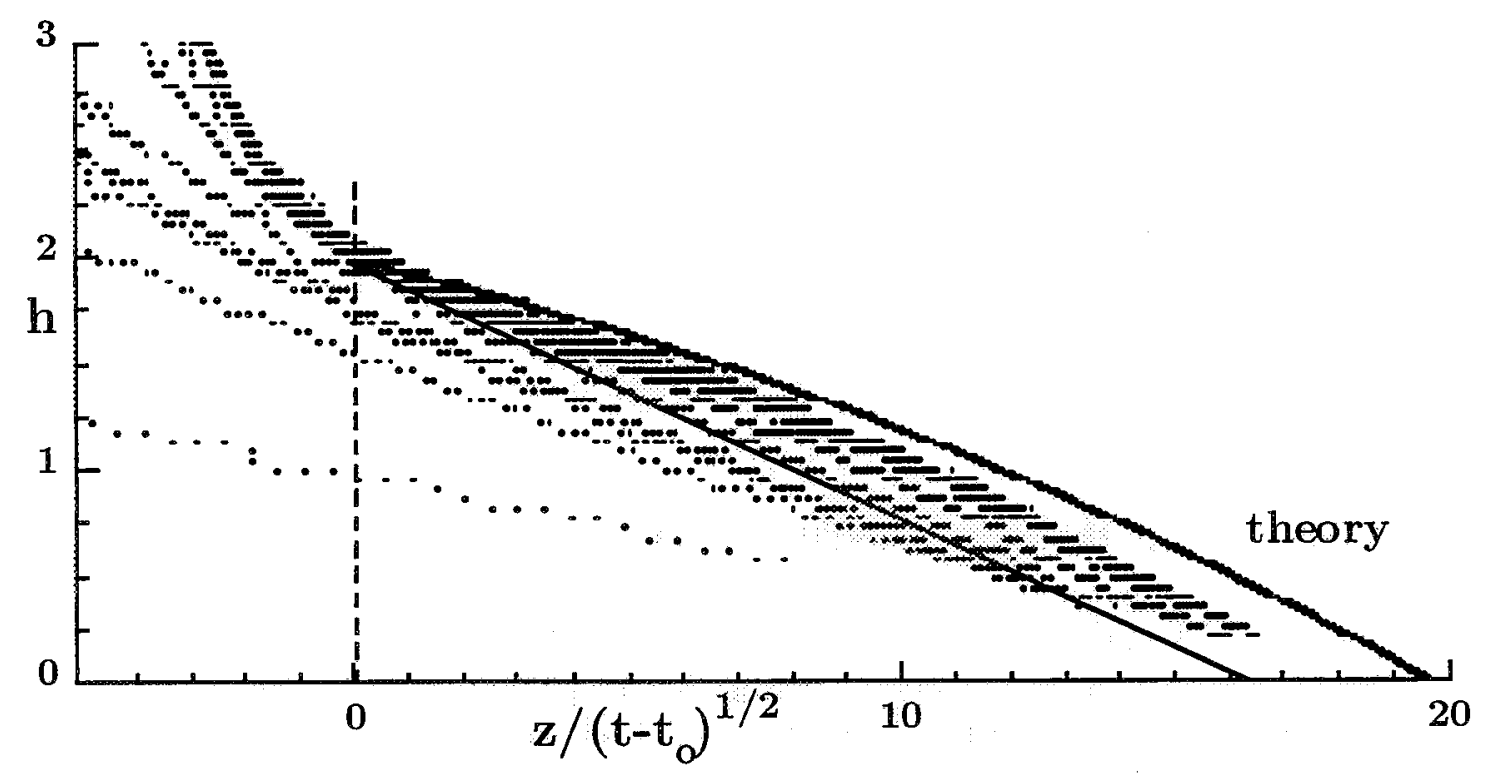

Figure 3.18: $h(\mathrm{~mm})$ as a furiction of $z /\left(t-t_{o}\right)^{1 / 2}\left(\mathrm{~mm} / \mathrm{s}^{1 / 2}\right)$ for T2 with $t_{o}=0.03 \mathrm{~s}$ providing tip collapse. Full theory provided along with linear tip solution. The first trace shown is at $t=0.0391 \mathrm{~s}$.

Recall that for comparison with the asymptotic analysis, $z$ is measured relative to $z_{H}$ and that for both $\mathrm{T} 1$ and $\mathrm{T} 2 t_{H} \simeq 0.4 s$. However, fluid flows into the corner $(z>0)$ prior to $t_{H}$ and so there is an initial volume of fluid within $z>0$ by the time $t=t_{H}$. This is the reason that the scaled traces of Figures $3.13 \mathrm{~b}$ and $3.17 \mathrm{~b}$ first overshoot and then converge towards the predicted behavior. As $t-t_{H}$ increases, the initial volume of fluid which was present in the corner at time $t_{H}$ constitutes a smaller portion of the total volume in the corner and the traces begin to show evidence of collapse about the predicted profile. The time necessary for complete collapse over the entire domain $z>0$ is presently uncertain, particularly in light of the fact that $t_{H} \simeq 0.4 s$ while the duration of the experiment was only about $2 \mathrm{~s}$.

Using $t_{o}$ as determined from the inertial reorientation time of the interface, the scaled traces of Figures 3.14 and 3.18 reveal collapse at the tip for $t \gtrsim 2 t_{o}$ and show

dry or were purposefully pre-wetted with a thin film of the test fluid prior to the test, the measurements of the surface profiles were statistically equivalent. This agreement is not common, but occurs for these tests because of the natural pre-wetting of the corner prior to the drop tests despite the presence of gravity. This is not the case for all cells, but for tests T1 and T2 the presence of a thin pre-wetting film along the corner should classify the entire family as employing the 'pre-wet' surface condition. 


\begin{tabular}{|c|c|c|c|c|c|}
\hline Test & Oh & $\begin{array}{c}1 \\
H^{2} / 8 \nu\end{array}$ & $\begin{array}{c}2 \\
10.7 \mu H / \sigma\end{array}$ & $\begin{array}{c}3 \\
\left(\rho R_{i c}^{3} / \sigma\right)^{1 / 2}\end{array}$ & $\begin{array}{c}4 \\
R_{i c}^{2} / 2 \nu\end{array}$ \\
\hline T1 & 0.008 & 0.8 & 0.004 & 0.12 & 10.5 \\
T2 & 0.05 & 0.05 & 0.05 & 0.05 & 0.6 \\
\hline
\end{tabular}

Table 3.3: The Ohnesorge number $\mathrm{O}$ and time constants, in seconds, for $\mathrm{T} 1$ and T2.

remarkable linearity for the traces taken at times $t<t_{H}$.

\subsubsection{Tip Solution Approximation}

Figures 3.14 and 3.18 show many surface profiles for which the height is nearly a linear function of $z /\left(t-t_{o}\right)^{1 / 2}$. These linear profiles are reminiscent of the leading order tip solution identified in Section 2.6.1 where $h=1-\eta / \sqrt{2}$ and $\eta_{\text {tip }}=\sqrt{2}$. This approximate solution is compared to the data for $\mathrm{T} 1$ and $\mathrm{T} 2$ on Figures 3.14 and 3.18 , respectively. The experimentally determined tip location is nearly bracketed by the full similarity and linear tip solutions. In fact, for test $\mathrm{T} 1$ which was of $1.01 \mathrm{~s}$ duration, the linear tip solution agrees well with the data while the full solution does not. That such agreement is observed for the linear tip solution is less likely due to coincidence than it is to the strong influence of the solution at the tip, especially prior to formation of a system length scale such as $H$. The flow velocities are highest at small times. As the column extends in length, the time for information to be propagated downstream from $z=0$ to the tip is $t \sim \mathcal{L}^{2}$. This creates a time lag between the origin $z=z_{H}$ and the tip. Consequently, though the constant height boundary condition is established at $t=t_{H}$, the tip will not respond to this condition until some time later. Thus, the tip region, for a time, acts independently of changes in conditions at $z=0$, and the tip solution is better suited for predictions of the flow at small times.

\subsubsection{Start-up Problem}

As typified in Figure 3.18 the linear surface profiles for $t<t_{H}$ increase in height, then for $t \simeq t_{H}$ the profiles collapse, and then give way to the predicted form of the similarity solution as $t$ increases above $t_{H}$. The fact that the linear profiles of these surfaces are reminiscent of other solutions to the governing similarity ODE (see Figure 2.8) makes one suspect that this 'start-up' problem might also be analyzed.

Recalling the transformation $h=\tau^{a} F(\eta)$, it is surprising that for $a=1 / 5$ a 


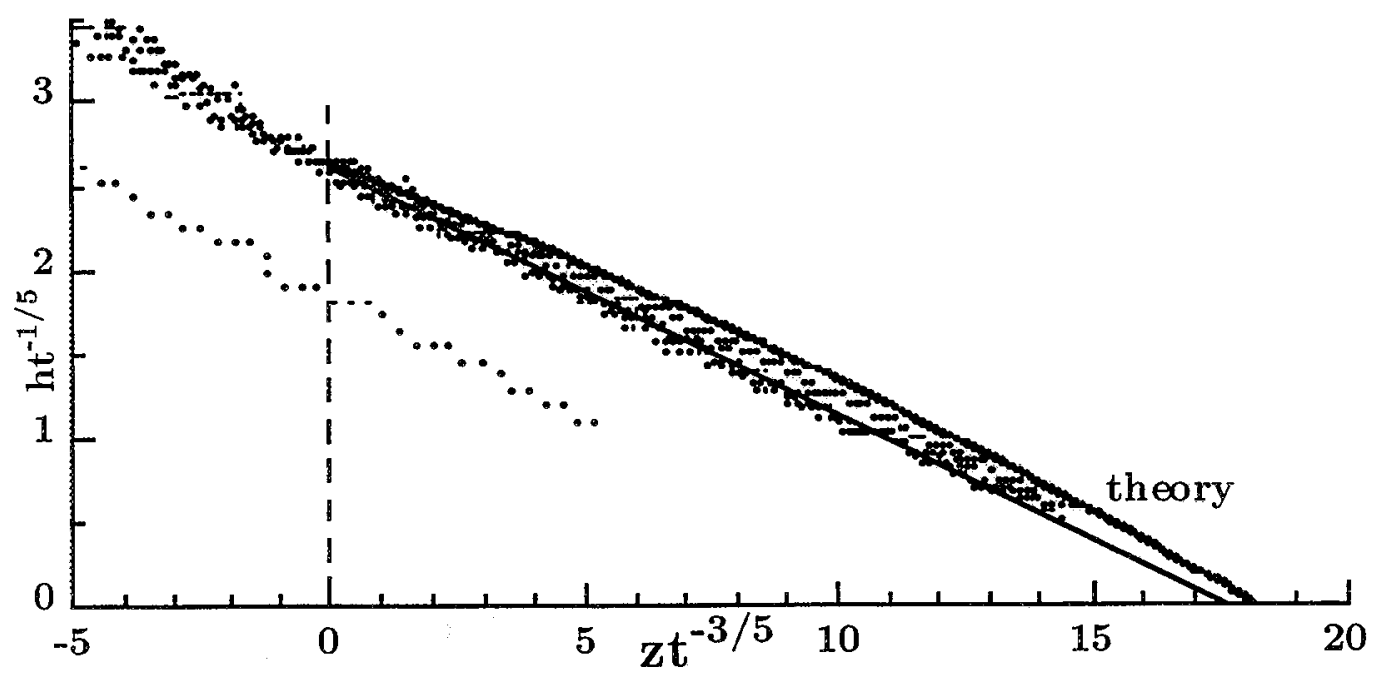

Figure 3.19: $h / t^{1 / 5}\left(\mathrm{~mm} / \mathrm{s}^{1 / 5}\right)$ as a function of $z / t^{3 / 5}\left(\mathrm{~mm} / \mathrm{s}^{3 / 5}\right)$ for $\mathrm{T} 2$ for the first five traces $\left(t<t_{H}\right)$ excluding the trace at $t=0$. The uncollapsed trace is for $t=0.039 \mathrm{~s}$ and $h / t^{1 / 5}=2.62$ at the origin. The full theoretical and linear tip solutions are provided.

favorable collapse of the surfaces for $t \lesssim t_{H}$ is observed for T2 as shown in Figure 3.19. The case of $a=1 / 5$ is coincidentally the case of constant flow at $\eta=0$ solved in Section 2.6.3, the exact solution of which is presented on the figure. ${ }^{4}$ A cause for the slight overprediction of the data by the theory could be attributed to dynamic contact effects, since velocities perpendicular to the contact line (and thus $\mathrm{Ca}$ ) are maximum during this portion of the flow (see Section 2.7.3). The value of the flow rate $\dot{Q}$ is necessary to quantify such effects as will be illustrated.

The linear tip solution for the constant flow problem also well predicts the surface profile during start-up as shown on Figure 3.19. Figure 3.20 plots $h\left(z_{i}, t\right)$ for $\mathrm{T} 2$ on a ln-ln scale for the $z_{i}$ nearest $z_{H}$ as determined by interpolation (as described in Section 3.4.1). The slope of $1 / 5$ is indicated as are the times for the first two surface traces appearing in Figure 3.18 and 3.19. The constant height time $t_{H}$ is also identified.

The fact that the initial transient of the constant height solution is well governed by, in this case, the constant flow problem, suggests that the time constants of the

\footnotetext{
${ }^{4}$ The condition of $a=1 / 5$ was not found to be true for all of the tests evaluated in this manner, but was found to vary somewhat with initial Bond number and fluid properties. The case of T2 is selected for ease of discussion.
} 


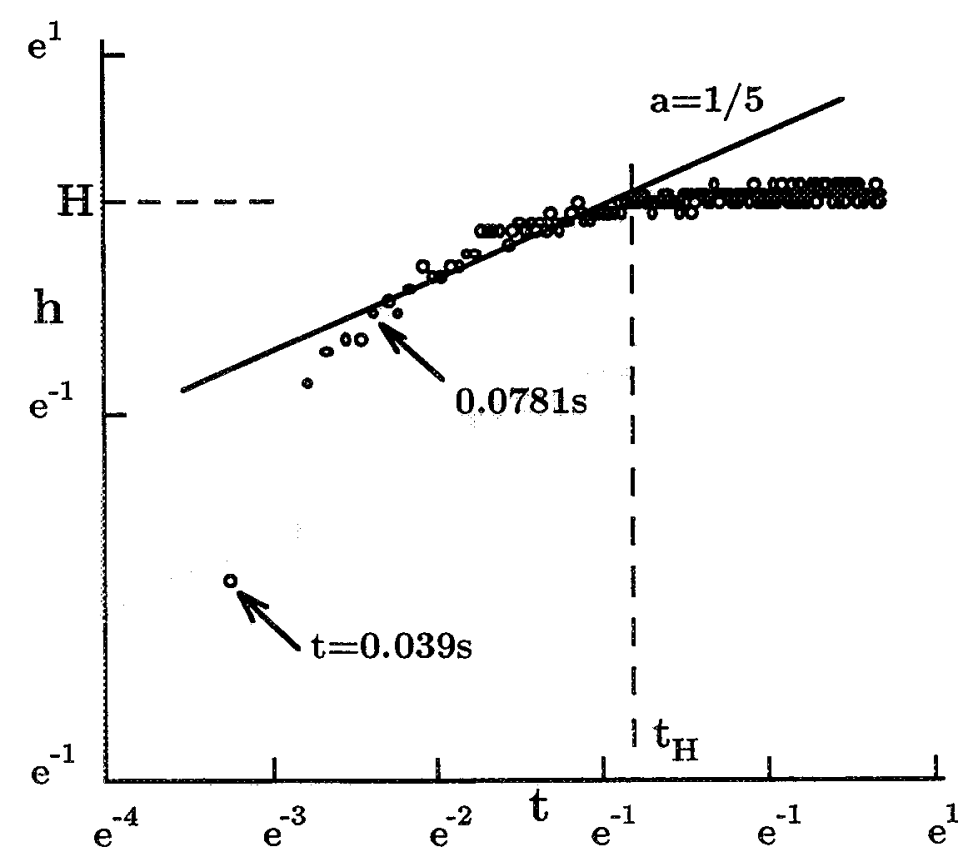

Figure 3.20: $h\left(z_{i}, t\right)(\mathrm{mm})$ as a function of $t(\mathrm{~s})$ for T2. Data taken at $z_{i} \simeq z_{H} . H$, $t_{H}$ and slope $a=1 / 5$ are indicated.

latter situation supersede in relevance those of the former. The time constants for the constant flow problem are listed in Table 3.4 for tests $\mathrm{T} 1$ and T2. The constants are functions of $\dot{Q}$ and $\dot{Q}_{T 1} \neq \dot{Q}_{T 2}$. The first constant is the viscous time scale (eq 2.125 ) while the second stems from the $\epsilon^{2} \ll 1$ constraint (eq 2.123). The latter is completely negligible in contrast to the former which may help to explain the quality of the agreement between the data and predictions in Figure 3.19. For T2 it is possible to estimate $\dot{Q}$ using $h / t^{1 / 5}=2.62\left(\mathrm{~mm} / \mathrm{s}^{1 / 5}\right)$ at $z=0$. This gives $\dot{Q} \simeq 7.8 \times 10^{-8}$ $\left(\mathrm{m}^{3} / \mathrm{s}\right)$ and the viscous time scale can be calculated to be $\simeq 1.5 \mathrm{~s}$. This implies that at $z=0$, the worst case, inertia is always present during the constant flow process. (Recall that the constant height condition is established at approximately $t \simeq 0.4 \mathrm{~s}$ for this test.) Inertial effects decay as $\sim h^{2}$ as the tip is approached which further vindicates the linear tip solution. An estimate of the capillary number using eq 2.130 with $\dot{Q}$ for T2 shows $C a_{c l} \simeq 5.3 \times 10^{-5} t^{-4 / 5}$ which, employing Hoffman's correlation eq 2.110, produces dynamic contact angle effects on the order $\theta_{d} \sim 20^{\circ}$, and $10^{\circ}$ at times $t=0.039$ and $0.4 \mathrm{~s}$, respectively. The impact of a change in effective contact angle of this magnitude is moderate and $f$ increases $14 \%$ for $\theta=\theta_{d}=20^{\circ}$ from its value at $\theta=\theta_{\text {stat }}=0$. The increase in $f$ (decrease in surface curvature) is not expected to decrease the tip velocity by more than $8 \%$ (see eq 2.114 adapted to the constant flow scenario). 


\begin{tabular}{|c|c|c|}
\hline Test & 1 & 2 \\
& $7.54\left(\mu \dot{Q}^{2} / \sigma\right)^{2 / 3} \nu^{-5 / 3}$ & $96.5(\mu / \sigma)^{3 / 2} \dot{Q}^{1 / 2}$ \\
\hline $\mathrm{T} 1$ & $5 \times 10^{9} \dot{Q}^{4 / 3}$ & $2.86 \dot{Q}^{1 / 2}$ \\
$\mathrm{~T} 2$ & $1 \times 10^{9} \dot{Q}^{4 / 3}$ & $30.6 \dot{Q}^{1 / 2}$ \\
\hline
\end{tabular}

Table 3.4: Time constants for $\mathrm{T} 1$ and $\mathrm{T} 2$, in seconds, as determined by constraints for the constant flow similarity solution.

\subsubsection{Further Remarks}

As discussed in Section 2.6.4 solutions to the asymptotic eq 2.73 at the tip are linear. Also, in the near tip region several of the modeling assumptions (e.g. low inertia and low $z$-curvature) are best satisfied. In fact, the linear tip solution does a better job of predicting the tip location for capillary rise than does the full solution at small times. These findings, along with the observation that the start-up problem for the capillary rise experiments may be governed by the transformation $h=\tau^{a} F(\eta)$, where $a$ for T2 was found to correspond to the constant flow similarity solution $(a=1 / 5)$, leads one to suspect that the problem of capillary rise might be dissected into start-up $\left(t<t_{H}\right)$ and constant height $\left(t \gg t_{H}\right)$ regimes. An intermediate regime must provide matching for the two solutions for times $t \sim t_{H}$. In this intermediate regime, the tip, acting (temporarily) independently of changes at the origin, maintains its dependence on the constant flow-like similarity solution for start-up while at the origin the constant height similarity profile is established which propagates downstream. An intermediate region is necessary to provide matching. Numerical solutions of the full Navier-Stokes equations would be helpful in this respect and would aid in identifying the regimes through which the flow transitions enroute to the $t^{1 / 2}$ dependence.

Since the capillary rise problem at small times is shared between a constant flowlike solution during start up, where $\mathcal{L} \sim t^{3 / 5}$, and the constant height solution, where $\mathcal{L} \sim t^{1 / 2}$, it is no wonder that $\mathcal{L} \sim t^{1 / 2}$ gives a reasonable representation of the tip location for all time! 


\section{Chapter 4}

\section{Conclusion}

\subsection{Summary of Results}

\subsubsection{Analytical Contributions}

In this work the governing equations describing capillary flows in containers with interior corners are analyzed under the constraints of a slender fluid column, slight surface curvature along the flow direction, low inertia, and low gravity. The quasisteady viscous streamwise flow is solved, averaged over the cross-sectional area, and using a local mass balance leads to a second order nonlinear diffusion equation for the height of the meniscus $h(z, t)$ as measured from the corner axis.

The unique velocity scale used in the nondimensionalization of the problem converts a numerical constant of $O(1)$ to $\infty$ used by other investigators [9][11][17] to an $O(1)$, tightly banded function $1 / 8 \lesssim F_{i} \leq 1 / 6$ for the shear free surface condition. This feature of the scaling clarifies the geometric influence of corner half-angle $\alpha$ and contact angle $\theta$ and shows that, in general, contact angle effects are manifest primarily through the alteration of the driving pressure gradient for the flow and not through significant changes in the cross-flow area. The banded nature of $F_{i}$ significantly lessens the dependence of the analysis on any numerically computed constant and, thus, the effects of container size, container geometry (including aspect ratio), and system contact angle, whether static or dynamic, may be assessed by inspection of the closed form results.

Two simple problems are considered first. The problem of a steady corner flow is solved to yield the maximum capillary pumping rate. Next, a solution is found for a perturbed fluid column of infinite extent. This solution yields a time constant 
which is useful in estimating settling times of disturbed systems. The geometric time constant $T$ is shown to increase with $\delta^{-1}$ as $\delta \rightarrow 0(\delta=\pi / 2-\alpha-\theta)$ and is minimized for $\alpha=30^{\circ}, \theta=0$. Gravity acting perpendicular to the corner axis is added to the infinite column analysis and the resulting solution provides the time response and stability limits of the perturbed system as a function of Bond number, $B o_{H}$.

The governing equation is then transformed into a general similarity form. For problems with an advancing tip, $h\left(z_{t i p}, t\right)=0$, an invariant transformation is employed which, by replacing one boundary condition, permits the use of a single step backwards Runge-Kutta technique when numerical solution is necessary. This method is accurate and efficient: it not only eliminates the need for iteration, but it also avoids the difficulties faced by other numerical techniques (i.e. forward shooting and two-point boundary value methods) in resolving the solution near the tip.

The generalized similarity equation is solved for a range of transform parameters, $a$ and $b$, and it is shown that a linear tip solution is valid near and at the tip for any choice of $a$. The high degree of inearity of the solutions is noted for $a \gtrsim 1 / 5$ and for $a=1$ an analytic solution is found which produces an exact linear surface profile. These linear-tending solutions provide insight into the start-up flow for the capillary rise experiments.

The similarity solution of Dong and Chatzis [17] for the case of a capillary flow in an initially empty corner, where at time $t=0^{+}$fluid is introduced such that the height of the meniscus $h(0, t)=H$ is fixed for all time, is re-formulated and solved. This is called the capillary rise problem in this study. The linear tip solution applied to this problem, though only appropriate in the near tip region, also produces the correct constant height condition at $z=0$. This feature is exploited in comparisons with the results of drop tower experiments for this problem. The analysis is extended by incorporating the influence of gravity perpendicular to the corner axis. The stability limit of the flow to an unfavorable orientation and magnitude of gravity is found to agree with that determined for the infinite column solution. The constant height condition is calculated using the approach of de Lazzer and Langbein [29] for the cases of $n$-sided regular polygonal and rectangular cross-sectioned containers. These results are applied to the experiments which employ test cells of like geometry.

New similarity solutions for a spreading drop (constant volume) and constant flow condition at $h(0, t)$ are found, the former yielding an exact analytic expression. A list of closed form expressions for key characteristics of the flows for the three similarity solutions is provided (Section 2.6.7). Observation of this list is useful for comparisons. For instance, the time dependence for the tip location $\mathcal{L}$ for each similarity solution is: capillary rise $\mathcal{L} \sim t^{1 / 2}$, spreading drop $\mathcal{L} \sim t^{2 / 5}$, and constant flow $\mathcal{L} \sim t^{3 / 5}$.

The constraints on each of the three similarity solutions are quantified and also 
listed for comparison (Section 2.7). The lubrication approximation demands a slender fluid column, the establishment of which requires a time scale that is proportional to the fluid viscosity $\mu$. On the other hand, the neglect of inertia depends on a viscous time scale which is inversely proportional $\mu$. The constraints of negligible curvature along the flow direction, gravity acting both perpendicular and parallel to the flow direction, dynamic contact angle effects at the moving contact line, and other problem-specific conditions are also considered.

\subsubsection{Experimental Contributions}

Experiments are outlined and test data is presented for the specific problem of capillary rise in transparent containers with interior corners after a step reduction in gravity. Test cells are employed which minimize optical distortion due to refraction. A $2.2 \mathrm{~s}$ drop tower is used to access the low-gravity environment. An extensive data set is collected over an unexplored range of flow parameters. Measurements reveal repeatability and accuracy, the role of inertia and column slenderness, and the effects of corner half-angle, container size, container aspect ratio, and fluid properties (with $\theta=0$ ). The locations of the advancing tip and global meniscus at the cell centerline are recorded as functions of time. An automated surface digitization technique is employed to measure surface profiles over the entire length of the column $h(z, t)$. It is shown that though some measurements correlate well with the predictions from the asymptotic analysis, the full surface profile measurements uncover discrepancies.

The initial transient due to the step reduction of gravity, characteristic of most drop tower tests, is governed by the inertial response time of the interface, $t_{o}$. For the irregular containers of this study, capillary surfaces are established when $t_{o} \simeq$ $0.75\left(\rho R_{i c}^{3} / \sigma\right)^{1 / 2}$, where $R_{i c}$ is the radius of the inscribed circle for the container crosssection. This result compares with the results of Siegert et al. [32] who study the response of fluid interfaces in containers of rotational symmetry. The time $t_{0}$ is useful in providing a time origin for the capillary dominated flow.

It is surprising that there exists a location of the interface which appears to remain stationary in time during the capillary rise experiments. This observation leads to the identification of a system origin where the constant height assumption for the similarity solution is applied. This solution in turn predicts the $t^{1 / 2}$ dependence for the flow. Specific experimental attention is paid to the establishment of this constant height condition and means for determining its validity and location are presented. The time $t_{H}$ required to establish this constant height is used as the time origin for comparisons of the similarity solution to the experimental results. It is shown that for times $t \gtrsim t_{H}$ the scaled surface profiles first overshoot and then collapse towards 
the similarity solution as $t$ increases. This 'overshoot' behavior is attributable to the fact that by the time $t_{H}$ a significant amount of fluid has already entered the corner. The period $t_{o} \lesssim t \lesssim t_{H}$ is the start-up period and for a special case (test $\mathrm{T} 2$, see Section 3.5.2) it is shown that the start-up flow is also largely governed by a similarity solution, but for the case of constant flow.

From such results it is observed that the initial transient experienced by the fluid is governed first by inertia $t \lesssim t_{o}$, then by a constant flow-like regime for $t_{o} \lesssim t \lesssim t_{H}$, where $h \sim t^{a}$, then by an intermediate regime which matches the tip profile (perhaps determined by a similarity solution as in the case of T2) to the constant height similarity profile extending from the origin, and finally by the constant height solution alone for $t \gg t_{H}$. For both the constant height and constant flow problems, solved in connection with the presentation of the data, the linear tip solution was found to give an adequate representation of the interface (slope and tip location) using the time origin of $t_{o}$. This success is due in part to the facts that the tip solution is the one initially established during start-up in the absence of a system length scale, that for $a \gtrsim 1 / 5$ all similarity profiles show a high degree of linearity, that a time lag exists $\left(t \sim \mathcal{L}^{2}\right)$ between changes in $h(0, t)$ and the tip, and that inertia and corneraxis curvature are negligible in the near tip region. Since the capillary rise problem at small times is shared between a constant flow-like solution, where $\mathcal{L} \sim t^{3 / 5}$, and the constant height solution, where $\mathcal{L} \sim t^{1 / 2}$, it is no wonder that $\mathcal{L} \sim t^{1 / 2}$ gives a reasonable representation of the tip location for all time! The linear tip solution is recommended for small times. The full similarity and linear tip solutions appear to bracket the measured behavior of the flow.

\subsection{Recommendations for Future Work}

The result of the velocity scale leading to $F_{i} \sim O(1)$ significantly lessens the dependence of the analysis on any numerically computed constant. Analytical investigations probing the added effects of dynamic wetting, free surface shear, surface tension gradients due to temperature or concentration gradients, and/or a participating overlaying fluid have a firm basis from which to proceed.

As is seen from surface traces of the developing flows during the capillary rise experiments, the general form of the similarity solution is matched at the tip where the effects of corner-axis curvature $\left(f \epsilon^{2} \ll 1\right)$ and inertia are negligible. Upstream at the apparent origin where the constant height condition is assumed applicable, these constraints are not readily satisfied and unpredicted corner-axis curvature remains throughout the tests. The influence of this curvature is central to the start-up 
problem where curvatures perpendicular and parallel to the corner-axis are of equal order. A numerical analysis incorporating the higher order, and presently neglected, curvature terms in the normal stress equation would eliminate the dependence of the solution on the constant height condition which is fortuitously, though incidentally, provided by the flow and not a true boundary condition for the governing system of equations. A complete numerical solution to the Navier-Stokes equations would add insight into the impact of inertia during the initial stages of the flow as well as identify the transition of the flow between certain power law regimes.

Experiments concerning spreading drops in open corners may provide an excellent data base for which to compare further analyses. Since for this flow scenario an exact analytical expression was found, the influence of first order inertia, dynamic contact angle, and/or corner-axis curvature may be considered in an asymptotic analysis.

\section{Acknowledgement}

Support for my research was provided by NASA's Microgravity Science and Application Division and the NASA Lewis Research Center.

I would like to thank Richard Vernon and Jack Salzman for encouraging me to pursue this work. Only through their concern and recommendation was this possible and I owe each a debt of thankfulness.

Concerning the problem of capillary flows in interior corners, I have been fortunate to have had collaborations with Paul Concus, Robert Finn, and Dieter Langbein, who have done much by way of definitive works in this field of research. The common enthusiasm and interests of Lloyd Trefethen and Andrew J. Bernoff were also well received and appreciated. I owe particular thanks to Seth Lichter, and as usual, I gladly acknowledge the clear and constant insights of my colleague R. Balasubramaniam.

I also thank Jennifer Kadlowec, Mike Luli, and Mark Pillar for providing assistance in the collection of the data. 


\section{Bibliography}

[1] Concus, P.; Finn, R. On Capillary free surfaces in the absence of gravity, Acta Math. 1974, 132, 177-198.

[2] Concus, P.; Finn, R. On capillary free surfaces in a gravitational field, Acta Math. 1974, 132, 207-223.

[3] Concus, P.; Finn, R. On the behavior of a capillary surface in a wedge, Appl. Math. Sci. 1969, 63(2), 292-299.

[4] Concus, P.; Finn, R. Dichotomous behavior of capillary surfaces in zero gravity, Microgravity Sci. Technol., III (1990), pp. 87-92; Errata, Microgravity Sci. Technol., III (1991), p. 230.

[5] Concus, P.; Finn, R. Capillary surfaces in a wedge-differing contact angles, Microgravity sci. technol. 1994, VII(2), pp 152-155.

[6] Mason, G.; Morrow, N. Capillary Behavior of a Perfectly wetting liquid in irregular triangular tubes, J. Colloid and Int. Sci. 1991, 141(1), 262.

[7] Wong, H.; Morris, S.; Radke, C.J. Three-dimensional menisci in polygonal capillaries, J. Colloid and Int. Sci. 1992, 148(2), 317-336.

[8] Concus, P.; Finn, R. In Low-Gravity Fluid Dynamics and Transport Phenomena, Koster, J.N.; Sani, R.L., Eds.; Progress in Astronautics and Aeronautics, AIAA, Vol. 130, 1990; pp 183-204.

[9] Ayyaswamy, P.S.; Catton, I.; Edwards, D.K. Capillary flow in triangular groves, J. of Appl. Mechanics 1974, 41, 332-336.

[10] Ma, H.B.; Peterson, G.P.; Lu, X. The influence of vapor-liquid interactions on the liquid pressure drop in triangular microgrooves, Int J. Heat Mass Transfer 1994, 37(15), 2211-2219. 
[11] Ransohoff, T.C.; Radke, C.J.; Laminar Flow of a Wetting Liquid along Corners of a predominantly Gas-Occupied Noncircular pore, J. Colloid and Int. Sci. 1988, 121(2), 392 .

[12] Ransohoff, T.C.; Gauglitz, P.A.; Radke, C.J. Snap-off of gas bubbles in smoothly constricted noncircular capillaries, AIChE J. 1987, 33(5), 753.

[13] Kolb, W.B.; Cerro, R.L. The motion of long bubbles in tubes of square cross section, Phys. Fluids A 1993, 5(7), 1549-1557.

[14] Legait, B. Laminar flow of two phases through a capillary tube with variable square cross-section, J. Colloid and Int. Sci. 1983, 96(1).

[15] Singhal, A.K.; Somerton, W.H. Two-phase flow through a noncircular capillary at low reynolds numbers, Presented at the 21st Annual Technical Meeting of the Petroleum Society of CIM, Calgary, May 1970.

[16] Columbus, R.L.; Palmer, H.J. "Architectured" fluid management of biological liquids, Clinical Chemistry 1987, 33(9), 1531-1537.

[17] Dong M.; Chatzis, I. The imbibition and flow of a wetting liquid along the corners of a square capillary tube, J. Colloid and Int. Sci., 1995, 172, 278-288.

[18] Langbein, D.; Grossbach, R.; Heide, W. Parabolic Flight Experiments on Fluid Surfaces and Wetting, Appl. Microgravity Tech. 1990, II(4), 198.

[19] Jaekle, D.E.Jr. Propellant management device conceptual design and analysis: vanes, Presented at the AIAA/SAE/ASME/ASEE 27th Joint Propulsion Conference, Sacramento, CA, June 1991; paper AIAA-91-2172.

[20] Rollins, J.R.; Grove, R.K.; Jaekle, D.E.Jr. Twenty three years of surface tension propellant management system design, development, manufacture, test, and operation, Presented at the AIAA-SAE-ASME-ASEE 21st Joint Propulsion Conference, Monterey, CA, July 1985; paper AIAA-85-1199.

[21] Langbein, D. The shape and stability of liquid menisci at solid edges, J. Fluid Mech. 1990, 213, 251-265.

[22] Dussan V, E.B. On the spreading of liquids on solid surface static and dynamic contact lines, Ann. Rev. Fluid Mech. 1979, 11, pp 371-400.

[23] Kistler, S.F. In Wettability; Berg, J.C., Ed.; Surfactant Science Series, 1993, Vol. 49, Chapter 6. 
[24] Kheshgi, H.S. Profile equations for films flows at moderate Reynolds numbers, AIChE J. 1989, 35(10), 1719-1727.

[25] Mayer, F.J.; McGrath, J.F.; Steele, J.W.; A class of similarity solutions for the nonlinear thermal conduction problem, J. Phys. A: Math. Gen., 1983, 16, 3393-3400.

[26] Lenormand, R., Zarcone, C., Role of roughness and edges during imbibition in square capillaries, SPE 13264, presented at the 59th Annual Meeting of the SPE, Houston, TX, Sept, 1984.

[27] Trefethen, L. unpublished results.

[28] White, F. Viscous Fluid Flow, McGraw-Hill, New York, 1974 (chpt. 3).

[29] de Lazzer, A.; Langbein, D. Krümmung des Meniskus im Prisma, Preprint, Nov. 1995, to be publisked.

[30] Hoffman, R.L. A study of the advancing interface. I. Interface shape in liquid-gas systems, J. Col. Inter. Sci. 1975, 50, p228

[31] Lekan, J.; Gotti, D.; Jenkins, A.j.; Owens. J.C.; Johnston, M.R. Users guide for the 2.2 second drop tower of the NASA Lewis Research Center, NASA TM 107090, April 1996

[32] Siegert, C.E.; Petrash, D.A.; Otto, E.W. Time response of liquid-vapor interface after entering weightlessness, NASA TN D-2458, 1964.

[33] Davis, S.H. Contact line problems in fluid mechanics, J. of Appl. Mechanics 1983, 50, pp 977-982. 


\section{Appendix A}

\section{Analytical Details}

\section{A.1 Equations and Boundary Conditions}

Governing system of equations for capillary dominated flow in an interior corner. All quantities are dimensional. See Figure 2.1 for coordinates and notation. The governing equations, following Davis [33], are the incompressible Navier-Stokes and continuity equations

$$
\begin{gathered}
\rho\left(\mathbf{v}_{t}+\mathbf{v} \cdot \nabla \mathbf{v}\right)=-\nabla P+\mu \nabla \cdot \underline{\mathbf{T}} \\
\underline{\mathbf{T}}=\nabla \mathbf{v}+(\nabla \mathbf{v})^{T} \\
\nabla \cdot \mathbf{v}=0
\end{gathered}
$$

where $\mathbf{v}=(u, v, w)$. A passive overlaying fluid is assumed and body forces are ignored. The boundary conditions for these equations are as follows: The no-slip condition is applied along the walls, $\mathbf{v}=0$ on $y= \pm x \tan \alpha$. At the free surface $S(x, y, t)$, the zero stress conditions are

$$
\begin{gathered}
\underline{\mathbf{T}} \cdot \mathbf{n} \cdot \mathbf{t}_{1}=\left(1+S_{z}^{2}\right)^{-1 / 2}\left(1+|\nabla S|^{2}\right)^{-1}\left[\left(1-S_{y}^{2}+S_{z}^{2}\right)\left(u_{y}+v_{x}-S_{z}\left(v_{z}+w_{y}\right)\right)\right. \\
\left.+2 S_{y}\left(u_{x}-v_{y}-S_{z}\left(u_{z}+w_{x}\right)-S_{z}^{2}\left(v_{y}+w_{z}\right)\right)\right]=0 \\
\underline{\mathbf{T}} \cdot \mathbf{n} \cdot \mathrm{t}_{2}=\left(1+S_{z}^{2}\right)^{-1 / 2}\left(1+|\nabla S|^{2}\right)^{-1 / 2}\left[\left(1-S_{z}^{2}\right)\left(u_{z}+w_{x}\right)-S_{y}\left(v_{z}+w_{y}\right)\right. \\
\left.+2 S_{z}\left(u_{x}-w_{z}\right)-S_{y} S_{z}\left(u_{y}+v_{x}\right)\right]=0
\end{gathered}
$$

where $t_{1}, t_{2}$, and $\mathbf{n}$ are outward unit tangents and unit normal to $S$ is given by

$$
\mathbf{n}=\left(1+|\nabla S|^{2}\right)^{-1 / 2}\left(1,-S_{y},-S_{z}\right)
$$




$$
\begin{gathered}
\mathbf{t}_{1}=\left(1+S_{z}^{2}\right)^{-1 / 2}\left(1+|\nabla S|^{2}\right)^{-1 / 2}\left(S_{y}, 1+S_{z}^{2},-S_{y} S_{z}\right) \\
\mathbf{t}_{2}=\left(1+S_{z}^{2}\right)^{-1 / 2}\left(S_{z}, 0,1\right)
\end{gathered}
$$

$\mathbf{t}_{2}$ is restricted to the plane $y=$ const. for convenience. The normal stress condition on $S$

$$
-P+\mu \underline{\mathbf{T}} \cdot \mathbf{n} \cdot \mathbf{n}=2 \mathcal{H} \sigma=\sigma \nabla \cdot \frac{\nabla S}{\left(1+|\nabla S|^{2}\right)^{1 / 2}}
$$

where the mean surface curvature, $\mathcal{H}$, is given by

$$
2 \mathcal{H}=\left(1+|\nabla S|^{2}\right)^{-3 / 2}\left(S_{y y}\left(1+S_{z}^{2}\right)+S_{z z}\left(1+S_{y}^{2}\right)-2 S_{y} S_{z} S_{y z}\right)
$$

and where

$$
\begin{gathered}
\underline{\mathbf{T}} \cdot \mathbf{n} \cdot \mathbf{n}=2\left(1+|\nabla S|^{2}\right)^{-1}\left(u_{x}-S_{y}\left(u_{y}+v_{x}\right)-S_{z}\left(u_{z}+w_{x}\right)\right. \\
\left.+S_{y} S_{z}\left(v_{z}+w_{y}\right)+S_{y}^{2} v_{y}+S_{z}^{2} w_{z}\right)
\end{gathered}
$$

with $|\nabla S|^{2}=S_{y}^{2}+S_{z}^{2}$. The two known boundary conditions on $S$ are $S=h$ and $S_{y}=0$ on $y=0$, and the two assumed conditions at the contact line $\left(y=y_{\max }\right)$ are $S=y_{\max } / \tan \alpha$ and

$$
\mathbf{n} \cdot \mathbf{k}=\left(1+|\nabla S|^{2}\right)^{-1 / 2}\left(\sin \alpha \pm S_{y} \cos \alpha\right)=\cos \theta
$$

The former is the interface wall intersection condition and the latter is the contact angle (static) condition. Here, $\mathbf{k}=(\sin \alpha, \mp \cos \alpha, 0)$.

\section{A.2 $F_{i}$ Details}

All solutions include at least terms up to second order for completeness. Recall

$$
\left\langle w_{o}\right\rangle=-F_{i} h_{z} \quad\left(\text { note } F_{i}>0\right)
$$

where

$$
F_{i}=\frac{2 f}{F_{A}} \int_{0}^{\sin \delta} \int_{\bar{y} / m}^{S_{o}} w_{o} d x d \bar{y}
$$

$F_{A}$ is the cross sectional area function and $y=\bar{y} / m, m=(\tan \alpha) / h f, \delta=\pi / 2-\alpha-\theta$, $\Omega=\pi / 2-\alpha, \phi=\delta / \Omega . \phi=1$ is the case of $\theta=0$.

$\underline{F_{I I}, \Omega^{2} \ll 1, \text { Free Surface }}$ 
Substituting $\Omega$ for $\alpha$ and dividing by $\cos ^{2} \alpha$, eq 2.29 becomes

$$
\frac{\partial \bar{P}_{o}}{\partial z}=\frac{\partial^{2} w_{o}}{\partial x^{2}}+\tan ^{2} \Omega \frac{\partial^{2} w_{o}}{\partial y^{2}}
$$

subject to $w_{0}=0$ on $x=y$ and

$$
\frac{\partial w_{o}}{\partial x}-\frac{\partial S_{o}}{\partial y} \frac{\partial w_{o}}{\partial y} \tan ^{2} \Omega=0
$$

on $x=S_{o}$. The pressure gradient term has been rescaled, $\bar{P}_{o}=P_{o} / \cos ^{2} \Omega$, and is $O(1)$. Expanding $w_{o}$ and $\tan ^{2} \Omega$ in powers of $\Omega^{2}$ yields

$$
w_{o}=w_{o_{o}}+\Omega^{2} w_{o_{1}}+\ldots
$$

and for eq A.12

$$
\frac{\partial \bar{P}_{o}}{\partial z}=\frac{\partial^{2} w_{o}}{\partial x^{2}}+\left(\Omega^{2}+\frac{2}{3} \Omega^{4}+\ldots\right) \frac{\partial^{2} w_{o}}{\partial y^{2}}
$$

with boundary conditions $w_{o}=0$ on $x=y$ and

$$
\frac{\partial w_{o}}{\partial x}-\left(\Omega^{2}+\frac{2}{3} \Omega^{4}+\ldots\right) \frac{\partial S_{o}}{\partial y} \frac{\partial w_{o}}{\partial y}=0
$$

on $x=S_{o}$. By Expanding $S_{o}$ and its derivatives in powers of $\Omega^{2}$, the system of eq 2.29 and its boundary conditions may be solved to $O\left(\Omega^{2}\right)$ giving

$$
\begin{gathered}
w_{o_{o}}=\bar{P}_{o_{z}}\left(\frac{x^{2}}{2}-\frac{y^{2}}{2}+h(y-x)\right) \\
w_{o_{1}}=w_{o_{o}}+\bar{P}_{o_{z}}\left[\frac{y k}{h}\left(2 y^{2}-x^{2}-x y\right)+\frac{k}{6 h}\left(x^{3}-3 x^{2} y+2 y^{3}\right)\right. \\
\left.+\left(4 y k+\frac{y^{2} k^{2}}{h^{2}}\left(y-\frac{3 h}{2}\right)-\frac{k h}{2}\right)(x-y)\right]
\end{gathered}
$$

where

$$
k=\phi\left(1-\frac{\phi}{2}\right)
$$

Note that these expansions are correct regardless of the sign of $\delta$ as long as $\delta^{2} \ll 1$ is maintained. In this way positively and negatively curved surfaces may be solved for cases of slight curvature. No solution of this nature is possible for $|\delta| \sim O(1)$. The average velocity for the case $\Omega^{2} \ll 1,\left\langle w_{o}\right\rangle_{I I}$, can now be determined to $O\left(\Omega^{2}\right)$ using

$$
\left\langle w_{o}\right\rangle_{I I}=\frac{2 \cot \Omega}{h^{2} F_{A}} \int_{0}^{y_{m}} \int_{y}^{S_{o}}\left(w_{o_{o}}+\Omega^{2} w_{o_{1}}\right) d x d y
$$


Performing the integration to $O\left(\Omega^{2}\right)$ produces

$$
\left\langle w_{o}\right\rangle_{I I}=-F_{I I} \frac{\partial h}{\partial z}
$$

where $F_{I I}$ is given by eq 2.45 .

\section{3. $F_{I I_{c}}, \Omega^{2} \ll 1$, Captive Surface}

The solution for $F_{I I_{c}}$ follows similarly as that for $F_{I I}$ above only the boundary conditions applicable to eq A.14 are $w_{o}=0$ on $x=y$ and $w_{o}=0$ on $x=S_{o}$. The zeroeth and first order solutions give

$$
\begin{gathered}
w_{o}=\frac{\bar{P}_{o_{z}}}{2}\left(x^{2}-x y-S_{o} x+S_{o} y\right) \\
w_{1}=\bar{P}_{o_{z}}\left[\frac{S_{o_{y y}}}{12}\left(x^{3}-x S_{o}^{2}-S_{o} x y-x y^{2}+y S_{o}^{2}+y^{2} S_{o}\right)\right. \\
\left.-\left(\frac{S_{o_{y}}}{2}-\frac{y S_{o_{y y}}}{4}\right)\left(x^{2}-x y-S_{o} x+S_{o} y\right)\right]
\end{gathered}
$$

Substitution of the above into the integral eq A.18 yields the full expression for $F_{I I_{c}}$ given by eq 2.46 .

\section{4. $F_{h y d}, F_{h y d_{c}}$, Hydraulic Diameter Approach, Free/Captive Surface}

The average velocity for the cross flow problem may also be estimated using the familiar hydraulic diameter approach in which

$$
\left\langle w_{o}\right\rangle_{h y d}=-\frac{R_{h y d}^{2}}{8 \mu} P_{o_{z}}
$$

where

$$
R_{\text {hyd }} \equiv \frac{2 A}{\mathcal{P}}
$$

$\mathcal{P}$ is the wetted perimeter. Determination of $A$ and $\mathcal{P}$ geometrically leads to the nondimensional result

$$
\left\langle w_{o}\right\rangle_{h y d}=-F_{h y d} \frac{\partial h}{\partial z}
$$

with

$$
F_{h y d}=\frac{1}{8}\left(\frac{F_{A}}{f \sin \delta}\right)^{2}
$$


and $F_{A}$ given by eq 2.35. For the captive surface condition the wetted perimeter, $\mathcal{P}$, is the entire perimeter of the cross flow area such that

$$
F_{h y d}=\frac{1}{8}\left(\frac{F_{A}}{f \sin \delta}\right)^{2}\left(\frac{1}{1+\frac{\delta \sin \alpha}{\sin \delta}}\right)^{2}
$$

\section{A.3 Similarity Details}

In this appendix the similarity parameters $a$ and $b$ are determined for the cases of (I) capillary rise, (II) spreading drop, and (III) constant flow.

I. Capillary Rise.

A mathematical treatment of the generalized similarity equations and applicable solutions is given by [25] in which the interest was nonlinear heat conduction. Specific details as applicable to the fluid mechanics problem are provided below. The desired boundary conditions for the capillary rise problem are $h(0, \tau)=1$ and $h(\mathcal{L}, \tau)=0$; the equation for $h(z, t)$ is further constrained by

$$
-\left.2 \int_{0}^{\tau}\left(h^{2} h_{z}\right)\right|_{z=0} d \hat{\tau}=\int_{0}^{\mathcal{L}} h^{2} d z
$$

Though the governing ODE is only second order, three conditions are necessary due to the introduction of the third unknown $\mathcal{L}(\tau)$. The first condition, that the height $h$ is fixed at $z=0$, is a conjecture given support by the experiments described in Section 3.4.1. The second boundary condition is the zero height condition at the meniscus tip. The final condition is an integral mass balance equation which states that the flow rate through the cross section at $z=0$ over time $\tau$ is equal to the volume of fluid in the corner from $z=0$ to the tip at $z=\mathcal{L}(t)$. By using the transforms of eq 2.59 and applying eq 2.61, the integraJ equation can be written as

$$
-\left.\frac{2 C_{1} C_{2}^{2}}{3 a+b+1}\left(F^{2} F_{\eta}\right)\right|_{\eta=0}=\int_{0}^{\eta_{t i p}} F^{2} d \eta
$$

It should be noted that the integral mass balance condition is always satisfied by any choice of $a$ and $b$ consistent with eq 2.61. To satisfy the condition $h(0, \tau)=1$, however, it is necessary to choose $a=0, b=-1 / 2$. With the selections $C_{1}=1$ and $C_{2}=2^{-1 / 2}$ eq $\mathrm{A} .28$ becomes

$$
\left.2\left(F^{2} F_{\eta}\right)\right|_{\eta=0}+\int_{0}^{\eta_{t i p}} F^{2} d \eta=0
$$

II. Spreading Drop 
For a drop of constant volume the boundary conditions are $h_{z}(0, \tau)=h(\mathcal{L}, \tau)=0$. The integral mass balance

$$
\int_{0}^{\mathcal{L}} h^{2} d z=\kappa
$$

states that the volume integral over $0 \leq z \leq \mathcal{L}$ is a constant $\kappa$ for all time. Substituting the similarity transforms of eq 2.59 into the above yields

$$
\frac{C_{1}^{2}}{C_{2}} \tau^{2 a-b} \int_{0}^{\eta_{t i p}} F^{2} d \eta=\kappa
$$

By applying eq 2.61 it can be shown that this equation yields similarity when $a=$ $-1 / 5$ and $b=-2 / 5$. For the selection of $C_{1}=\left(\kappa^{2} / 5\right)^{1 / 5}$ and $C_{2}=\left(5^{2} \kappa\right)^{-1 / 5}$ eq A.31 simplifies to

$$
\int_{0}^{\eta_{t i p}} F^{2} d \eta=1
$$

$\eta_{t i p}$ can be determined directly by substituting the exact solution for $F$ into the above integral (or by substituting values for $F^{+}$, etc.).

III. Constant Flow

As for the spreading drop problem, the similarity parameters $a$ and $b$ are determined from the integral mass balance. For this case

$$
\int_{0}^{\mathcal{L}(\tau)} h^{2} d z=2 q \tau
$$

which states that the constant flow rate through the cross sectional area at $z=0$ at time $\tau$ is equal to the volume of fluid in the domain $0 \leq z \leq \mathcal{L}(\tau)$. Substituting from eq 2.59 yields

$$
\frac{C_{1}^{2}}{C_{2}} \tau^{2 a-b-1} \int_{0}^{\eta_{t i p}} F^{2} d \eta=2 q
$$

Applying eq 2.61 it can be shown that similarity is achieved with $a=1 / 5$ and $b=-3 / 5$. For $C_{1}=\left(5 q^{2}\right)^{1 / 5}$ and $C_{2}=\left(5^{3} q\right)^{-1 / 5}$, the equation can be written as

$$
\int_{0}^{\eta_{t i p}} F^{2} d \eta=\frac{2}{5}
$$

\section{A.4 Similarity with $g_{x}$}

The influence of gravity in the $x$-direction can be incorporated into the analysis. In the dimensional problem the pressure needs only to be modified as

$$
P_{o}^{\prime}=-\frac{\sigma}{h^{\prime} f}+\rho g h^{\prime}
$$


so that

$$
P_{o_{z}}^{\prime}=\left(\frac{\sigma}{h^{\prime 2} f}+\rho g\right) h_{z}^{\prime}
$$

In nondimensional terms this produces

$$
P_{o_{z}}=\left(\frac{1}{h^{2}}+B o_{H}\right) h_{z}
$$

so that

$$
\left\langle w_{o}\right\rangle=-F_{i}\left(1+h^{2} B o_{H}\right) h_{z}
$$

Substitution of this result into eq 2.23 noting $h^{2} \propto A$ and $\tau \equiv F_{i} t / 2$ yields eq 2.75 . For the infinite column problem, solving for $P_{o_{z}}$ in a similar manner and by expanding $h=h_{o}+\epsilon h_{1}$ and taking $h_{o}=$ const yields eq 2.58 . 


\section{Appendix B}

\section{Correction for Optical Distortion}

An approximate correction to optical distortions attributable to mismatched indices of refraction between the cell material $\left(N_{D_{1}}\right)$ and test fluid $\left(N_{D_{2}}\right)$ could be made by a ray trace analysis. Assuming the surface $S(y, h(z, t))$ is a portion of a circle in the $x-y$ plane, a simple relationship may be determined between the true value of $h$ and that of the apparent $h$ measured experimentally, here labeled $h_{\text {exp }}$. The sketch of Figure B.1 is appropriate for the long working distances of these experiments. Knowing the slope and intercept for the line segment $O-P$, the conditions of its tangential intersection with $S_{o}$ (see eq 2.32) produce the relationship

$$
h=h_{\exp } \frac{1+\tan \gamma \tan \alpha}{1+f-f \sec \gamma}
$$

where

$$
\gamma=\alpha-\sin ^{-1}\left(\frac{N_{D_{1}} \sin \alpha}{N_{D_{2}}}\right)
$$

Optical distortion increases with increasing $\alpha$ and for the capillary rise test a maximum was experienced for the square acrylic cell, $N_{D_{1}}=1.491$, and the $0.65 \mathrm{cs}$ PDMS fluid, $N_{D_{2}}=1.374$. This worst case gives $h=0.919 h_{\text {exp }}$, approximately a $10 \%$ correction. For the same fluid in an equilateral triangle a correction of $5 \%$ results and many test corrections were $3 \%$ and below. 


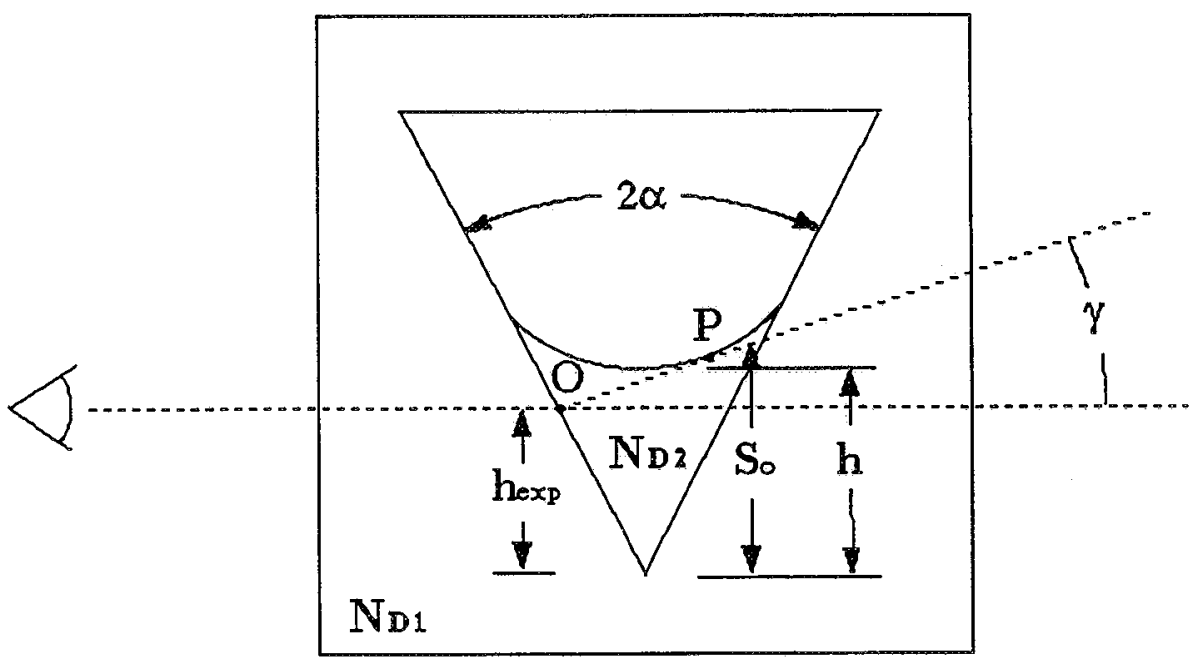

Figure B.1: Schematic of ray trace correction for $h(z, t)$ measurements. 
Public reporting burden for this collection of information is estimated to average 1 hour per response, including the time for reviewing instructions, searching existing data sources,

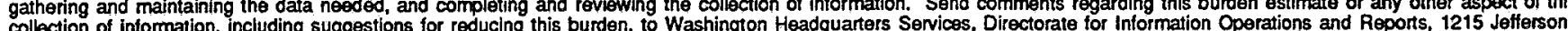
Davis Highway, Suite 1204. Arlington, VA 22202-4302, and to the Office of Management and Budget, Paperwork Reduction Project (0704-0188), Washington, DC 20503.

\begin{tabular}{|l|c|c|}
\hline 1. AGENCY USE ONLY (Leave blank) & $\begin{array}{c}\text { 2. REPORT DATE } \\
\text { November } 1996\end{array}$ & $\begin{array}{r}\text { 3. REPORT TYPE AND DATES COVERED } \\
\text { Technical Memorandum }\end{array}$ \\
\hline
\end{tabular}

\section{TITLE AND SUBTTRE}

Capillary Flow in an Interior Corner

6. AUTHOR(S)

Mark Milton Weislogel

7. PERFORMING ORGANIZATION NAME(S) AND ADDRESS(ES)

National Aeronautics and Space Administration

Lewis Research Center

Cleveland, Ohio 44135-3191

9. SPONSORING/MONITORING AGENCY NAME(S) AND ADDRESS(ES)

National Aeronautics and Space Administration

Washington, DC 20546-0001
5. FUNDING NUMBERS

WU-962-24-00
REPORT NUMBER

E-10526
10. SPONSORING/MONITORING AGENCY REPORT NUMBER

NASA TM-107364

11. SUPPLEMENTARY NOTES

This report was submitted as a dissertation in partial fulfillment of the requirements for the degree Doctor of Philosophy to Northwestern University, Evanston, Illinois, June 1996. Responsible person, Mark Milton Weislogel, organization code $6712,(216) 433-2877$.

12a. DISTRIBUTION/AVAILABILITY STATEMENT

Unclassified - Unlimited

Subject Categories 28 and 34

This publication is available from the NASA Center for AeroSpace Information, (301) 621-0390.

13. ABSTRACT (Maximum 200 words)

The design of fluids management processes in the low-gravity environment of space requires an accurate model and description of capillarity-controlled flow in containers of irregular geometry. Here we consider the capillary rise of a fluid along an interior corner of a container following a rapid reduction in gravity. The analytical portion of the work presents an asymptotic formulation in the limit of a slender fluid column, slight surface curvature along the corner, small inertia, and low gravity. New similarity solutions are found and a list of closed form expressions is provided for flow rate and column length. In particular, it is found that the flow is proportional to $\mathrm{t}^{1 / 2}$ for a constant height boundary condition, $\mathrm{t}^{2 / 5}$ for $\mathrm{a}$ spreading drop, and $\mathrm{t}^{3 / 5}$ for constant flow. In the experimental portion of the work, measurements from a $2.2 \mathrm{~s}$ drop tower are reported. An extensive data set, collected over a previously unexplored range of flow parameters, includes estimates of repeatability and accuracy, the role of inertia and column slendemess, and the effects of comer angle, container geometry, and fluid properties. Comprehensive comparisons are made which illustrate the applicability of the analytic results to low-g fluid systems design.

\begin{tabular}{|l}
\hline $\begin{array}{l}\text { 14. SUBJECT TERMS } \\
\text { Capillary flow; Wetting; Drop tower, Spreading; Corner; W }\end{array}$ \\
\begin{tabular}{|l|c|} 
17. SECURTY CLASSIFICATION \\
OF REPORT \\
Unclassified
\end{tabular} \\
\hline
\end{tabular}

19. SECURITY CLASSIFICATION
OF ABSTRACT
Unclassified

15. NUMBER OF PAGES
101
16. PRICE CODE
A06

20. LIMTTATION OF ABSTRACT 
